\title{
ASPHERICITY AND FINITENESS FOR CERTAIN GROUP PRESENTATIONS
}

\author{
Martin Edjvet and Noha Eljamel \\ School of Mathematical Sciences, University of Nottingham, \\ University Park, Nottingham NG7 2RD, UK
}

\begin{abstract}
We study diagrammatic reducibility for the relative group presentations $\mathcal{R}_{n}(k, l, \varepsilon)=\langle H, x|$ $\left.t^{3} x^{k} t^{2} x^{\varepsilon(k+l)}\right\rangle$ where $H=\left\langle t \mid t^{n}\right\rangle, n \geq 7, k \geq 1, l \geq 0$ and $\varepsilon= \pm 1$. We apply our results to classify finiteness for the group $G_{n}(k, l, \varepsilon)$ defined by $\mathcal{R}_{n}(k, l, \varepsilon)$ apart from the two exceptional cases $(n, k, l, \varepsilon)=(7,2,1,-1)$ and $(9,1,1,-1)$.
\end{abstract}

Keywords: ashericity; relative group presentation; picture; relative diagram; curvature; diagrammatically reducible.

2010 Mathematics subject classification: 20F05; 57M05.

\section{Introduction}

In this paper we consider the group presentations

$$
\mathcal{P}_{n}(k, l, \varepsilon)=\left\langle x, t \mid t^{n}, t^{3} x^{k} t^{2} x^{\varepsilon(k+l)}\right\rangle
$$

where $n \geq 4, k \geq 1, l \geq 0$ and $\varepsilon= \pm 1$. Our motivation for studying $\mathcal{P}_{n}(k, l, \varepsilon)$ and the groups $G_{n}(k, l, \varepsilon)$ they define is based principally on the work of Bogley and Williams in [8] where they consider $n=4$ and $n=6$; and also to some extent on the work of Pride in [20] that applies to $n=5$. Indeed, if we consider finiteness then necessary and sufficient conditions for $G_{n}(k, l, \varepsilon)$ being finite are given for $n=4$ and $n=6$ in [8] and for $n=5$ in [20] so it is natural to consider what occurs when $n \geq 7$ and it is this we do here. Combining our results with those from [8] and [20] yields Theorems 1.2 and 1.3 below which show that finiteness has been decided in all but two outstanding cases.

A further property we study is the following. The presentation $\mathcal{P}_{n}(k, l, \varepsilon)$ can be considered as a relative group presentation

$$
\mathcal{R}_{n}(\varepsilon)=\mathcal{R}_{n}(k, l, \varepsilon)=\left\langle H, x \mid t^{3} x^{k} t^{2} x^{\varepsilon(k+l)}\right\rangle
$$

where $H=\left\langle t \mid t^{n}\right\rangle$. Relative presentations have been the subject of much study and the reader is referred to [5]; in particular, when the relator, as in this case, has free product length four the question of asphericity has been considered [1-4,6-9,11,13,14,17,21].

Following [5] we define a relative presentation $\mathcal{R}$ to be (weakly) diagrammatically reducible if every connected (strictly) spherical picture over $\mathcal{R}$ contains a dipole. See Section 2 for a discussion of these terms, although we note here that (weakly) diagrammatically reducible for $\mathcal{R}$ coincides with (weakly) aspherical as defined in [7] and, since the relator is not a proper power, implies (weakly) aspherical as defined in [5] ( see [5, Theorem 3.4]).

Necessary and sufficient conditions for diagrammatic reducibility of $\mathcal{R}_{4}(k, l, \varepsilon)$ and $\mathcal{R}_{6}(k, l, \varepsilon)$ are given in [8, Theorem A]; and it can be deduced from [7] and [20] (or see [5, Theorem 4.5]) that $\mathcal{R}_{5}(k, l, \varepsilon)$ is never diagrammatically reducible. This again motivates us to consider $n \geq 7$. Our main result is the following, the proof of which forms the bulk of this paper.

Theorem 1.1 If any of the following conditions holds then $\mathcal{R}_{n}(k, l, \varepsilon)$ is weakly diagrammatically reducible: 
(i) $\varepsilon=1, l \geq 1$ and $n \geq 7$;

(ii) $\varepsilon=-1, l \geq 1$ and either $n=11$ or $n \geq 13$;

(iii) $\varepsilon=-1, l \geq 1, l \neq k$ and $n \in\{9,10,12\}$;

(iv) $\varepsilon=-1, l \geq 1, l \neq k, l \neq 2 k, k \neq 2 l$ and $n \in\{7,8\}$.

Assume that one of the conditions of Theorem 1.1 holds. Then since the natural map from $H$ to $G_{n}(k, l, \varepsilon)$ is injective for $n \geq 7[16,18]$, it follows that $\mathcal{R}_{n}(k, l, \varepsilon)$ is diagrammatically reducible and then that every finite subgroup of $G_{n}(k, l, \varepsilon)$ is conjugate to a subgroup of $H$ [7]. This fact together with some preliminary results from Section 2 is used to prove the next two theorems. We restate here that for the two theorems below, the cases $n=4$ and $n=6$ are dealt with in [8] and $n=5$ in [20] so this paper focuses on $n \geq 7$. We mention further that Theorem 1.3(iv) relies on Theorem 3.6 in [5].

Theorem 1.2 The group $G_{n}(1)=G_{n}(k, l, 1)$ is finite if and only if one of the following conditions holds:

(i) $n=4$ and $l=0$, in which case $G_{n}(1)$ has order $8 k$;

(ii) $n=4, l \neq 0$ and $(k, l)=1$, in which case $G_{n}(1)$ has order

$$
4(2 k+l)\left(2^{k+l}+1-2^{k+1}(\sqrt{2})^{l} \cos (l \pi / 4)\right) ;
$$

(iii) $n=5$ and $(k, l)=1$, in which case $G_{n}(1)$ has order $5\left(k^{5}+(k+l)^{5}\right)$;

(iv) $n=6$ and $l=0$, in which case $G_{n}(1)$ has order $12 k$;

(v) $n=6, l \neq 0$ and $(k, l)=1$, in which case $G_{n}(1)$ has order

$$
6(2 k+l)\left(3^{2 k+l}+4^{2 k+l}-2^{2 k+l+1}(\sqrt{3})^{2 k+l} \cos (l \pi / 6)\right) ;
$$

(vi) $n \geq 7, l=0$ and either $k=1$ or $(n, 5)=1$, in which case $G_{n}(1)$ is cyclic of order $2 k n$.

When $\varepsilon=-1$ there are the exceptional cases $G_{7}(2,1,-1)$ and $G_{9}(1,1,-1)$ (also an exceptional case in [13]) whose orders remains an open problem.

Theorem 1.3 Let $G_{n}(-1)=G_{n}(k, l,-1)$ and assume that $(n, k, l) \neq(7,2,1)$ or $(9,1,1)$. Then $G_{n}(-1)$ is finite if and only if one of the following conditions holds:

(i) $n=4 ; l \neq 0$ and $(k, l)=1$, in which case $G_{n}(-1)$ has order

$$
4 l\left(2^{l}+1-2(\sqrt{2})^{l} \cos ((2 k+1) \pi / 4)\right) ;
$$

(ii) $n=5, l \neq 0$ and $(k, l)=1$, in which case $G_{n}(-1)$ has order $5\left((k+l)^{5}-k^{5}\right)$;

(iii) $n=6, l \neq 0$ and $(k, l)=1$, in which case $G_{n}(-1)$ has order

$$
6 l\left(3^{l}+4^{l}-2(2 \sqrt{3})^{l} \cos ((2 k+1) \pi / 6)\right) ;
$$

(iv) $n=7$ and $k=l=1$, in which case $G_{n}(-1)$ has order 203 .

The proof of Theorem 1.1 is given in Section 3; and the proofs of Theorems 1.2 and 1.3 are in Section 4 .

\section{Diagrams and preliminary results}

The reader is referred to [5] and [7] for definitions of many of the basic terms used in this section. 


\subsection{Diagrams}

The proof of Theorem 1.1(i) follows from [3, Theorem 1.1] so we consider only $\mathcal{R}_{n}(-1)$. Suppose by way of contradiction that one of the conditions in Theorem 1.1(ii)-(iv) holds and that the relative presentation

$$
\mathcal{R}_{n}(-1)=\left\langle H, x \mid t^{3} x^{k} t^{2} x^{-(k+l)}\right\rangle
$$

where $H=\left\langle t \mid t^{n}\right\rangle, n \geq 7, k \geq 1$ and $l \geq 1$ is not weakly diagrammatically reducible, that is, there exists a reduced connected strictly spherical picture $\boldsymbol{P}$, say, over $\mathcal{R}_{n}(-1)$.

Recall that such a spherical picture is a finite collection of pairwise disjoint discs (called vertices) in the interior of a disc together with a collection of pairwise disjoint simple arcs that meet the vertices transversely in their end points; and, since it is spherical, the arcs do not meet the boundary of the ambient disc. There is a well-defined labelling for pictures. In our case each arc of $\boldsymbol{P}$ is equipped with a normal orientation and labelled by $x$; each oriented corner of $\boldsymbol{P}$ is labelled $t^{i}$ where $i \in\{0,2,3\}$; reading the labels clockwise on the corners and arcs at any given vertex yields $t^{3} x^{k} t^{2} x^{-(k+l)}$ (up to cyclic permutation and inversion); and, given that $\boldsymbol{P}$ is strictly spherical, the product of the sequence of corner labels read in an anti-clockwise traversal of any given region of $\boldsymbol{P}$ yields the identity in the group $H=\left\langle t \mid t^{n}\right\rangle$.

Now delete the boundary of $\boldsymbol{P}$ and let $\boldsymbol{K}$ denote the dual of $\boldsymbol{P}$ with the labelling of $\boldsymbol{K}$ inherited from that of $\boldsymbol{P}$. Then $\boldsymbol{K}$ is a strictly spherical diagram over $\mathcal{R}_{n}(-1)$ and observe that $\boldsymbol{K}$ forms a tessellation of the sphere. Each oriented corner of $\boldsymbol{K}$ has label $t^{i}$ where $i \in\{0,2,3\}$; each oriented edge has label $x$; the sum of the powers of $t$ read around any vertex of $\boldsymbol{K}$ is congruent to 0 modulo $n$; and the product of the sequence of corner labels and edge labels read in a clockwise direction of any given region of $\boldsymbol{K}$ yields $t^{3} x^{k} t^{2} x^{-(k+l)}$. The degree of a vertex $v$ of $\boldsymbol{K}$, denoted $d(v)$, is the number of edges of $\boldsymbol{K}$ incident at $v$. The degree of a region $\Delta$ of $\boldsymbol{K}$, denoted $d(\Delta)$, is the number of vertices of $\Delta$ of degree $>2$. If $v$ is a vertex of $\boldsymbol{K}$ then $l(v)$, the label of $v$, is the cyclic word obtained from reading the corner labels at $v$ in a clockwise direction and is considered up to cyclic permutation and inversion. Moreover, $l(v)=1$ in $H$ for each vertex $v$.

We adopt the notation

$$
a x \lambda_{1}^{-1} x \ldots x \lambda_{k-1}^{-1} x b x^{-1} \lambda_{k} x^{-1} \ldots x^{-1} \lambda_{2 k+l-2} x^{-1}
$$

for $t^{3} x^{k} t^{2} x^{-(k+l)}$. Therefore $a=t^{3}, b=t^{2}$ and $\lambda_{i}=t^{0}=1(1 \leq i \leq 2 k+l-2)$. An example of a vertex in $\boldsymbol{P}$ when $k=2, l=1$ is given in Figure 2.1(i); and the corresponding dual

region in $\boldsymbol{K}$ in Figure 2.1(ii). More generally, each oriented region $\Delta$ of $\boldsymbol{K}$ is given (up to cyclic permutation and inversion) by Figure 2.1(iii). For ease of presentation, when drawing regions of $\boldsymbol{K}$ we omit the arrows and edge labels, the vertices of degree 2 , use $\bar{a}, \bar{b}$ to denote $a^{-1}, b^{-1}$ and use $\lambda, \bar{\lambda}$ to denote $\lambda_{i}, \lambda_{i}^{-1}$ where $1 \leq i \leq 2 k+l-2$. For example there is (up to cyclic permutation and inversion) one region of $\boldsymbol{K}$ of degree 3, and two regions of degree 4 and these are shown in Figure 2.1(iv)-(vi). We will for example use the notation $\left(u_{1}, u_{2}\right)$-edge to denote the edge between vertices $u_{1}$ and $u_{2}$ in Figure 2.1(v); using the corner labels we will also refer to the same edge as an $(a, b)$-edge of the region.

The following assumptions can be made without any loss of generality.

(A1) $\boldsymbol{K}$ is minimal with respect to number of regions.

(A2) Subject to (A1), $\boldsymbol{K}$ is maximal with respect to number of vertices of degree 2 .

Note that (A1) implies that there are no vertex sublabels of the form $a w a^{-1}, a^{-1} w a, b w b^{-1}$, $b^{-1} w b, \lambda_{i} w \lambda_{i}^{-1}, \lambda_{i}^{-1} w \lambda_{i}$ where $w=1$ in $H$. For otherwise a bridge move [10] followed by 


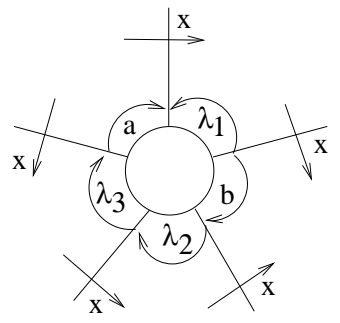

(i)

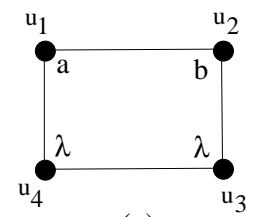

(v)

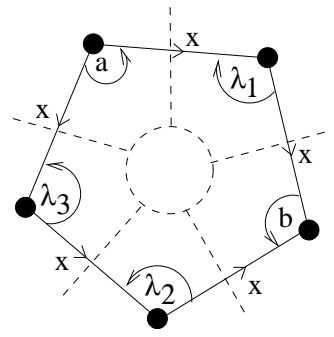

(ii)

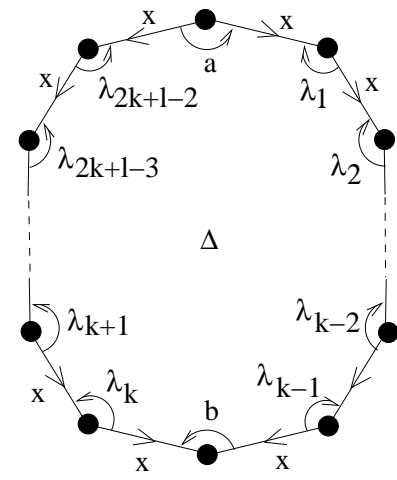

(iii)

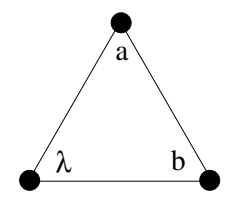

(iv)

Figure 2.1: vertices of $\boldsymbol{P}$; regions of $\boldsymbol{K}$; bridge moves; and the star graph $\Gamma$

cancellation of inverse regions would yield a diagram over $\mathcal{R}_{n}(-1)$ with fewer regions. Note however we allow $\lambda \lambda^{-1}$ or $\lambda^{-1} \lambda$ to be a vertex sublabel with the understanding that $\lambda=\lambda_{i}$ and $\lambda^{-1}=\lambda_{j}^{-1}(i \neq j)$.

Lemma 2.1 (i) If $v$ is a vertex of $\boldsymbol{K}$ and $l(v)=\left(\lambda \lambda^{-1}\right)^{k}$ or $\left(\lambda^{-1} \lambda\right)^{k}$ then $k=1$; (ii) it can be assumed without any loss of generality that $a \lambda \lambda^{-1} a$ is not a vertex sublabel.

Proof (i) This is Lemma 3.1 in [14]. (ii)Suppose that such a label does occur, as shown in Figure 2.1 (vii). Let $l_{i}$ denote the number of edges between the vertex $u_{i}$ and $u(i=1,2)$. Then by making $\min \left(l_{1}, l_{2}\right)$ bridge moves starting at the vertex $u$, we can obtain a new diagram with the same number of regions and vertices of degree 2 but, since $\theta_{i} \neq a^{ \pm 1}(i=1,2)$ in Figure 2.1(vii), with one fewer sublabel of the form $a \lambda \lambda^{-1} a$.

\subsection{The star graph}

The star graph $\Gamma$ of $\mathcal{R}_{n}(-1)$ is given by Figure 2.1(viii) with the understanding that the edge labelled $\lambda$ represents the $2 k+l-2$ edges labelled $\lambda_{i}$. Each vertex label in $\boldsymbol{K}$ yields a closed path $\Gamma$. Using this fact, Lemma 2.1 and previous comments a routine check yields the following result.

Lemma 2.2 (i) If $d(v) \leq 5$ then $l(v)$ is one of the following (up to cyclic permutation and inversion).

$$
\begin{array}{ll}
d(v)=2: \lambda \lambda^{-1} & d(v)=5: a^{5}(n=15) \\
d(v)=3: a^{3}(n=9) & d(v)=5: b^{5}(n=10) \\
d(v)=4: a^{4}(n=12) & d(v)=5: a^{2} \lambda b \lambda^{-1}(n=8) \\
d(v)=4: b^{4}(n=8) & d(v)=5: a \lambda b^{2} \lambda^{-1}(n=7)
\end{array}
$$


(ii) If $d(v)=6$ and $n \in\{7,8,9,10,12,15\}$ then $l(v)$ is one of the following.

$$
\begin{array}{llll}
a^{3} \lambda b^{-1} \lambda^{-1} & (n=7) & a^{2} \lambda b^{2} \lambda^{-1} & (n=10) \\
b^{4} \lambda^{-1} \lambda & (n=8) & b^{6} & (n=12) \\
a \lambda b^{3} \lambda^{-1} & (n=9) & &
\end{array}
$$

(iii) If $d(v)=7$ and $n \in\{7,8,9,10,12,15\}$ then $l(v)$ is one of the following.

$$
\begin{array}{llll}
a^{2} \lambda b^{-3} \lambda^{-1} & & a^{2} \lambda b \lambda^{-1} \lambda \lambda^{-1} & (n=8) \\
a \lambda \lambda^{-1} \lambda b^{2} \lambda^{-1} & (n=7) & a^{3} \lambda \lambda^{-1} \lambda \lambda^{-1} & (n=9) \\
a \lambda b \lambda^{-1} \lambda b \lambda^{-1} & (n=7) & a^{4} \lambda b^{-1} \lambda^{-1} & (n=10) \\
a \lambda b^{2} \lambda^{-1} \lambda \lambda^{-1} & (n=7) & b^{5} \lambda^{-1} \lambda & (n=10) \\
a^{2} \lambda \lambda^{-1} \lambda b \lambda^{-1} & (n=8) & a^{2} \lambda b^{3} \lambda^{-1} & (n=10)
\end{array}
$$

\subsection{Curvature}

An angle function for a given diagram is a real-valued function on the set of the corners. If $d(v)=d$ then $\frac{2 \pi}{d}$ is assigned to each corner at $v$. This way the curvature of a vertex will be 0 ; and the curvature $c(\Delta)$ of a region $\Delta$ of degree $m$ whose vertices have degree $d_{i}>2(1 \leq i \leq m)$ is given by

$$
c(\Delta)=c\left(d_{1}, \ldots, d_{m}\right)=(2-m) \pi+2 \pi \sum_{i=1}^{m} \frac{1}{d_{i}} .
$$

Using, for example, Euler's formula, it follows that for a connected spherical diagram the total curvature of is $\sum c(\Delta)=4 \pi$. We aim to achieve a contradiction by showing that for the diagram $\boldsymbol{K}$, any positive curvature that exists in $\boldsymbol{K}$ can be sufficiently compensated by the negative curvature thereby showing that $4 \pi$ cannot be attained. To this end we define a distribution scheme (see, for example, [12]), that is, we locate each $\Delta$ satisfying $c(\Delta)>0$ and distribute $c(\Delta)$ to near regions $\hat{\Delta}$ of $\Delta$ in a way that will be made precise. Once the distribution scheme has been defined, for such regions $\hat{\Delta}$ define $c^{*}(\hat{\Delta})$ to equal $c(\hat{\Delta})$ plus all the positive curvature $\hat{\Delta}$ receives minus all the curvature $\hat{\Delta}$ distributes.

Lemma 2.3 $\mathcal{R}_{n}(-1)$ is weakly diagrammatically reducible if one of the following holds: $c(\Delta) \leq 0(\forall \Delta \in K)$; (ii) $c^{*}(\hat{\Delta}) \leq 0$ for each $\hat{\Delta}$ that receives positive curvature.

Proof If (i) holds then $c(\boldsymbol{K})=\sum c(\Delta) \leq 0$; or if (ii) holds then $c(\boldsymbol{K}) \leq \sum c^{*}(\hat{\Delta}) \leq 0$ and each case yields the desired contradiction.

\subsection{Preliminary results}

Recall that $G_{n}(\varepsilon)=G_{n}(k, l, \varepsilon)$ is the group defined by $\mathcal{P}_{n}(\varepsilon)$ and that $n \geq 7$.

Lemma 2.4 The group $G_{n}(1,1,-1)$ is infinite for $n \in\{8,10,12\}$, is finite of order 203 for $n=7$ and has order $>9$ for $n=9 ; G_{7}(2,1,-1)$ has order $>7$ and $G_{8}(2,1,-1)$ is infinite; and $G_{n}(1,2,-1)$ is infinite for $n \in\{7,8\}$. In particular, none of these groups are cyclic.

Proof The result follows from calculations made in $\operatorname{GAP}[15]$. In detail, $G_{8}(1,1,-1)$ has a subgroup of index 10 with infinite Abelianisation; applying the Newman infinity criterion[19] at prime $\mathrm{p}=2,2,3$ (respectively) to the second derived subgroup shows that $G_{10}(1,1,-1), G_{12}(1,1,-1)$, $G_{8}(2,1,-1)$ (respectively) is infinite; the second derived subgroup of $G_{7}(1,2,-1)$ has infinite Abelianisation; $G=G_{8}(1,2,-1)$ has a subgroup of index 9 whose core has derived subgroup D of index 36864 in $\mathrm{G}$ and applying the Newman infinity criterion at prime $\mathrm{p}=2$ to $\mathrm{D}$ shows that 


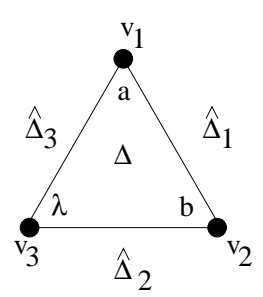

(i)

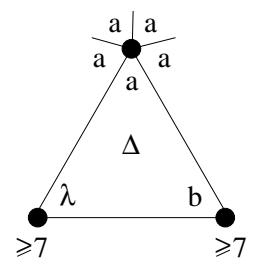

(ii)

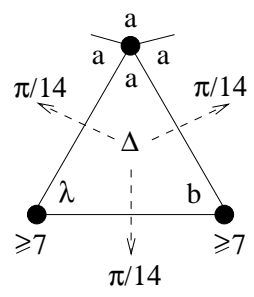

(iii)

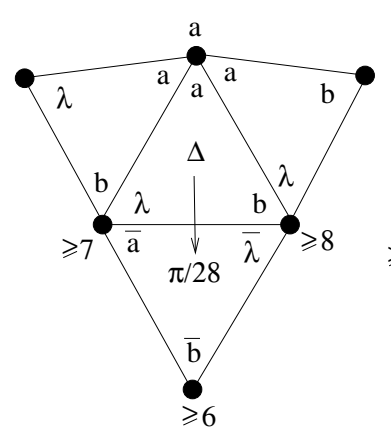

(iv)

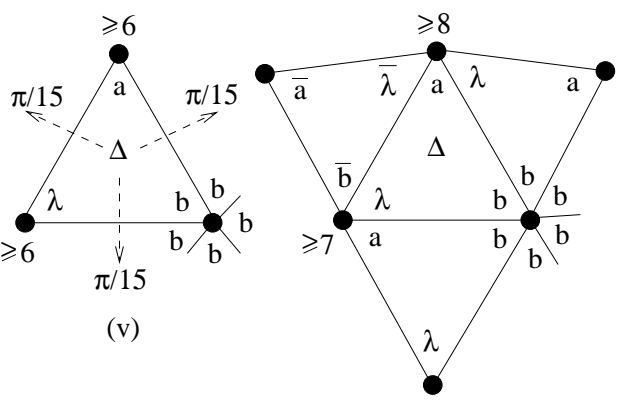

(vi)

Figure 3.1: the region $\Delta$ and it's neighbours for $n \in\{10,12,15\}$

$\mathrm{G}$ is infinite; the second derived subgroup of $G_{9}(1,1,-1)$ has index 333; and the second derived subgroup of $G_{7}(2,1,-1)$ has index 56 .

Lemma 2.5 If $l=k>1 G_{n}(-1)$ is infinite for $n \in\{7,8,9,10,12\}$; and if $k=2 l>2$ or $l=2 k>2$ then $G_{n}(-1)$ is infinite for $n \in\{7,8\}$.

Proof If $l=k>1$ then $G_{n}(-1)$ is the amalgamated free product $G_{n}(-1)=\left(\left\langle y, t \mid t^{n}, t^{3} y t^{2} y^{-2}\right\rangle\right.$ $\left.*\left\langle x \mid x^{u k}\right\rangle ; y=x^{k}\right)$; if $k=2 l>2$ then $G_{n}(-1)=\left(\left\langle y, t \mid t^{n}, t^{3} y^{2} t^{2} y^{-3}\right\rangle *\left\langle x \mid x^{u l}\right\rangle ; y=x^{l}\right)$; or if $l=2 k>2$ then $G_{n}(-1)=\left(\left\langle y, t \mid t^{n}, t^{3} y t^{2} y^{-3}\right\rangle *\left\langle x \mid x^{u k}\right\rangle ; y=x^{k}\right)$. Either $|y|=\infty$ in which case put $u=0$; otherwise put $u=|y|$. Since $k>1$ and $l>1$ it follows from Lemma 2.4 that $G_{n}(-1)$ is infinite.

Lemma 2.6 If $n \geq 7$ then $G_{n}(k, 0,1)$ is finite if and only if either $k=1$ or $(n, 5)=1$. Moreover, if $G_{n}(k, 0,1)$ is finite then it is cyclic of order $2 k n$.

Proof

$$
\begin{aligned}
G_{n}(k, 0,1) & =\left\langle x, t \mid t^{n}, t^{3} x^{k} t^{2} x^{k}\right\rangle \\
& =\left\langle x, t, y \mid t^{n}, t^{3} x^{k} t^{2} x^{k}, y^{-1} t^{2} x^{k}\right\rangle \\
& =\left\langle x, t, y \mid t^{n}, t y^{2}, y^{-1} t^{2} x^{k}\right\rangle \\
& =\left\langle x, y \mid y^{2 n}, y^{-5} x^{k}\right\rangle
\end{aligned}
$$

If $k=1$ the $G_{n}(1,0,1)=\left\langle y \mid y^{2 n}\right\rangle$ is cyclic of order $2 n$; if $k>1$ and if $(n, 5)>1$ then $G_{n}(k, 0,1)$ maps onto $C_{5} * C_{k}$ and is infinite; or if $k>1$ and $(n, 5)=1$ then $G_{n}(k, 0,1)=\left\langle x \mid x^{2 k n}\right\rangle$ is cyclic of order $2 k n$.

\section{Proof of Theorem 1.1}

The proof of Theorem 1.1(i) follows from the main theorem in [3] so we need only consider Theorem 1.1(ii)-(iv). Thus as discussed in Section 2 we assume $\boldsymbol{K}$ to be a reduced connected 
strictly spherical diagram over $\mathcal{R}_{n}(-1)$. It suffices to show that condition (i) or (ii) of Lemma 2.3 holds. Recall that $n \geq 7, k \geq 1$ and $l \geq 1$. We proceed according to the value of $n$. In what follows much use will be made of Lemma 2.2 , often without explicit mention.

\section{$3.1 n \geq 10$}

If $n \notin\{10,12,15\}$ then $d(v) \geq 6$ for each vertex $v$ of degree $>2$ by Lemma 2.2(i) and it follows from equation $(2.1)$ that $c(\Delta) \leq 0$ for each region $\Delta$ of $\boldsymbol{K}$; or if $n \in\{10,12,15\}$ and $d(\Delta) \geq 4$ then $c(\Delta) \leq 0$ by Lemma 2.2(i) and equation (2.1). So if $c(\Delta)>0$ then $d(\Delta)=3$ and $\Delta$, the vertices of $\Delta$ and neighbouring regions are given by Figure 3.1(i).

Let $n=15$. Given that $c(\Delta)>0$, we must have $d\left(v_{1}\right)=5$ and $\Delta$ is given by Figure 3.1(ii). But now $d\left(v_{2}\right) \geq 7$ and $d\left(v_{3}\right) \geq 7$ by Lemma 2.2 and $c(\Delta) \leq c(5,7,7)<0$.

Let $n=12$ and assume that $l \neq k$. If $c(\Delta)>0$ then $\Delta$ is given by Figure 3.1(iii) and $c(\Delta) \leq$ $c(4,7,7)=\frac{\pi}{14}$. If $d\left(\hat{\Delta}_{i}\right)=3(1 \leq i \leq 3)$ then (since $\left.l \neq k\right) \Delta$ is given by Figure 3.1(iv), in which case add $c(\Delta) \leq c(4,7,8)=\frac{\pi}{28}$ to $c\left(\hat{\Delta}_{2}\right)$ as shown; otherwise add $c(\Delta) \leq \frac{\pi}{14}$ to $c\left(\hat{\Delta}_{i}\right)$ where $\hat{\Delta}_{i}$ is the first of $\hat{\Delta}_{1}, \hat{\Delta}_{2}, \hat{\Delta}_{3}$ such that $d\left(\hat{\Delta}_{i}\right)>3$ as indicated in Figure 3.1 (iii). This completes the distribution scheme for $n=12$. Suppose that $\hat{\Delta}$ receives positive curvature. If $d(\hat{\Delta})=3$ then $\hat{\Delta}=\hat{\Delta}_{2}$ of Figure 3.1(iv) and $c^{*}(\hat{\Delta}) \leq c(6,7,8)+\frac{\pi}{28}<0$; or if $d(\hat{\Delta})=q>3$ then $c^{*}(\hat{\Delta}) \leq c(4,6, \ldots, 6)+q\left(\frac{\pi}{14}\right)<0$. Let $n=10$ and assume that $l \neq k$. If $c(\Delta)>0$ then $\Delta$ is given by Figure 3.1(v). If $d\left(\hat{\Delta}_{i}\right)=3(1 \leq i \leq 3)$ then $\Delta$ is given by Figure 3.1(vi) but then $c(\Delta) \leq c(5,7,8)<0$; otherwise add $c(\Delta) \leq c(5,6,6)=\frac{\pi}{15}$ to $c\left(\hat{\Delta}_{i}\right)$ for some $i$ as in the case $n=12$ (see Figure $3.1(\mathrm{v})$ ). This completes the distribution scheme for $n=10$. Let $\hat{\Delta}$ receive positive curvature. Then $d(\hat{\Delta})=q>3$ and $c^{*}(\hat{\Delta}) \leq c(5,6, \ldots, 6)+q\left(\frac{\pi}{15}\right)<0$.

\section{$3.2 n=9(l \neq k)$}

If $d(v) \leq 7$ then $l(v) \in\left\{\lambda \lambda^{-1}, a^{3}, a \lambda b^{3} \lambda^{-1}, a^{3} \lambda \lambda^{-1} \lambda \lambda^{-1}, a^{2} \lambda b^{-3} \lambda^{-1}\right\}$ by Lemma 2.2 so if $c(\Delta)>$ 0 then $d(\Delta)=3$ and $\Delta$ is given by Figure $3.1(\mathrm{i})$ in which $d\left(v_{2}\right) \geq 6$ and $d\left(v_{3}\right) \geq 6$. The distribution scheme for $n=9$ is given by Figures 3.2-3.3. Proceeding according to $d\left(\hat{\Delta}_{i}\right)(1 \leq$ $i \leq 3)$, we give a brief explanation for each case below. Note that it can always be assumed without any loss that $c(\Delta)$ takes the maximum possible value. Note also that use will be made of the assumption $l \neq k$, often without explicit mention.

$d\left(\hat{\Delta}_{i}\right)>3(1 \leq i \leq 3)$ : Figure $3.2(\mathrm{i})$-(iii). Distribute $c(\Delta)$ to $c\left(\hat{\Delta}_{1}\right)$ and $c\left(\hat{\Delta}_{3}\right)$ according to $d\left(v_{2}\right)$ and $d\left(v_{3}\right)$ as shown. Note that $c(3,7,7)=\frac{5 \pi}{21}, c(3,6,7)=\frac{2 \pi}{7}$ and $c(3,6,8)=\frac{\pi}{4}$; and that $d\left(v_{3}\right)=6$ forces $d\left(v_{2}\right) \geq 8$.

$d\left(\hat{\Delta}_{i}\right)=3, d\left(\hat{\Delta}_{j}\right)>3, j \neq i$ : Figure 3.2(iv)-(xvi). Observe that $l \neq k$ is used, for example, to force the corner label $\lambda^{-1}$ in (vi). Distribute $c(\Delta)$ according to $d\left(v_{2}\right)$ and $d\left(v_{3}\right)$ as shown. Note that $c(3,7,8)=\frac{17 \pi}{84}$ and $c(3,8,8)=\frac{\pi}{6}$. There will be three exceptions to these rules and they occur in Figure 3.3(v), (vii) and (viii) and will be explained below.

$d\left(\hat{\Delta}_{1}\right)>3, d\left(\hat{\Delta}_{2}\right)=d\left(\hat{\Delta}_{3}\right)=3$ : Figure $3.2($ xvii) and (xviii). Distribute $c(\Delta)$ according to $d\left(v_{3}\right) \geq 8$ or $=7$ as shown.

$d\left(\hat{\Delta}_{1}\right)=d\left(\hat{\Delta}_{2}\right)=3, d\left(\hat{\Delta}_{3}\right)>3$ : Figure 3.3(i)-(viii). Here $d\left(v_{2}\right) \geq 6$ and $d\left(v_{3}\right) \geq 7$. Distribute $c(\Delta)$ according to $d\left(v_{2}\right)$ and $d\left(v_{3}\right)$. Observe that in Figure $3.3(\mathrm{v})$, (vii) and (viii) curvature is distributed from $\Delta$ to $\hat{\Delta}_{3}$ across the $(\lambda, a)$-edge of $\hat{\Delta}_{3}$ via $\hat{\Delta}_{1}$; and that $c\left(\hat{\Delta}_{1}\right)$ is distributed differently from the rule given in Figure $3.2(x i)$, (xiii), (xiii) respectively.

$d\left(\hat{\Delta}_{1}\right)=d\left(\hat{\Delta}_{3}\right)=3, d\left(\hat{\Delta}_{2}\right)>3$ : Figure 3.3(ix)-(xiii). Distribute $c(\Delta)$ to $c\left(\hat{\Delta}_{2}\right)$ according to $d\left(v_{2}\right)$ and $d\left(v_{3}\right)$ as shown. 


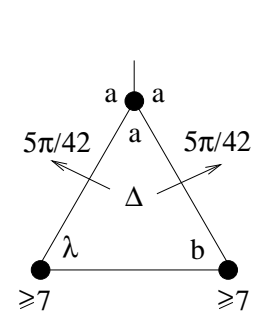

(i)

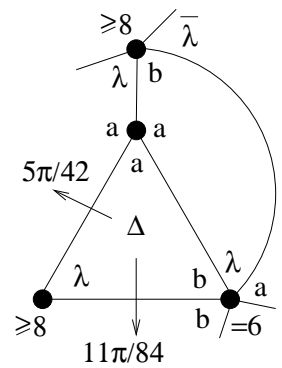

(vi)

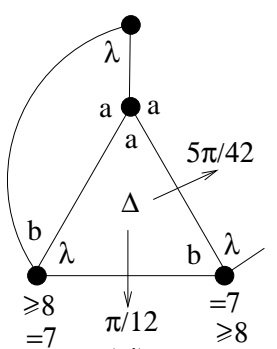

(xi)

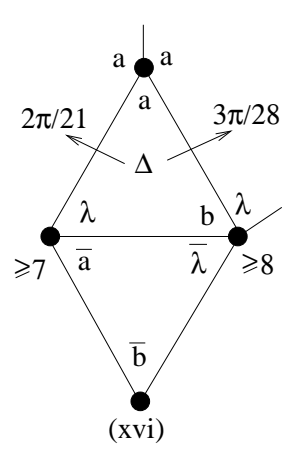

(xvi)

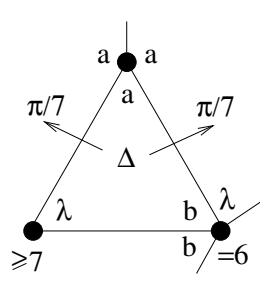

(ii)

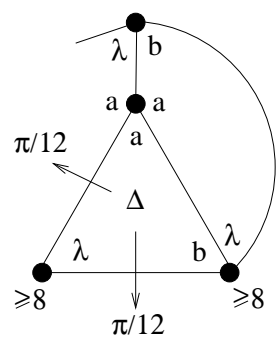

(iv)

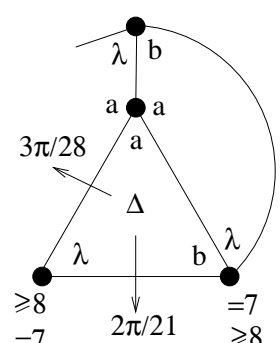

(v)

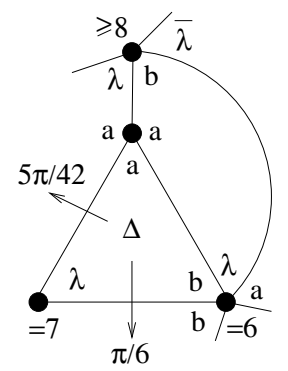

(vii)

(iii)

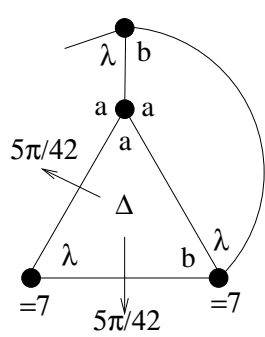

(ix)

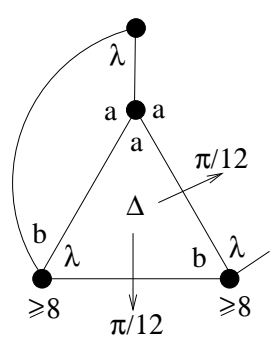

(x)
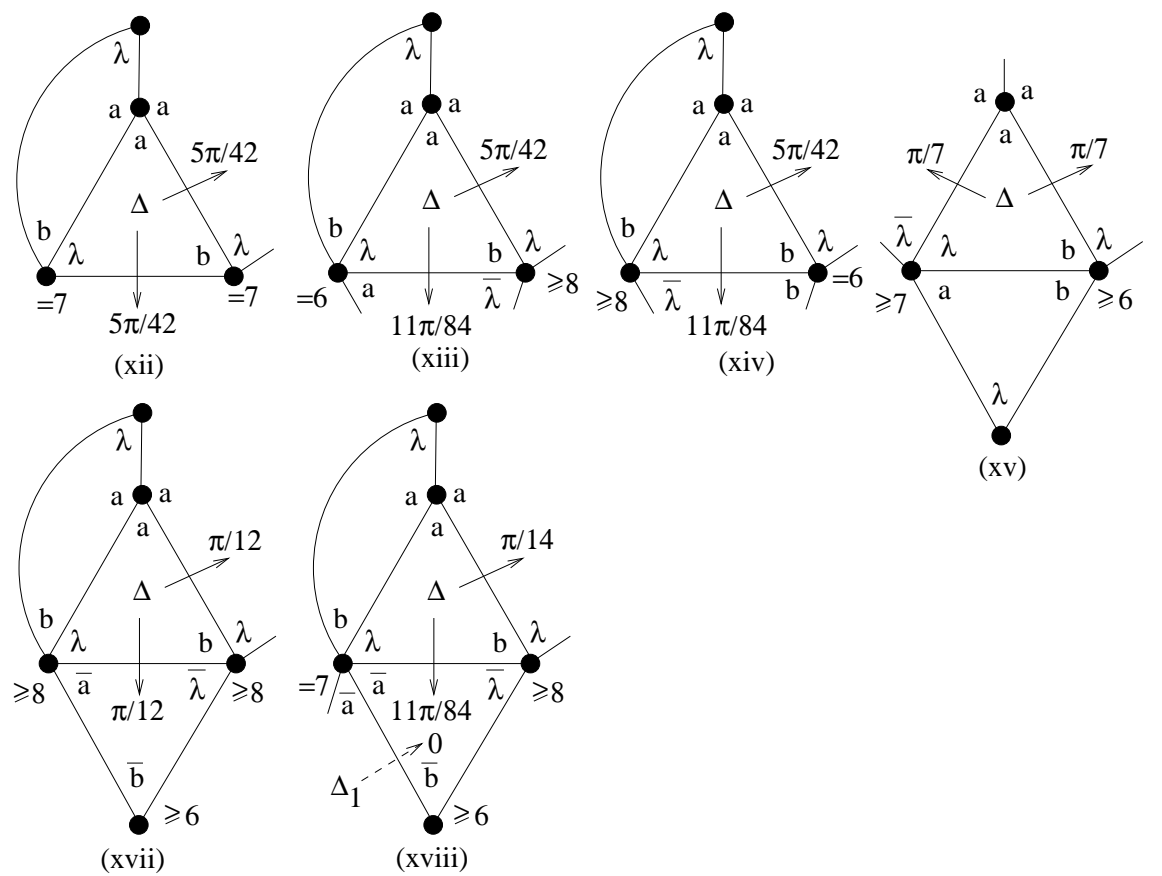

Figure 3.2: distribution of curvature for $n=9$ 


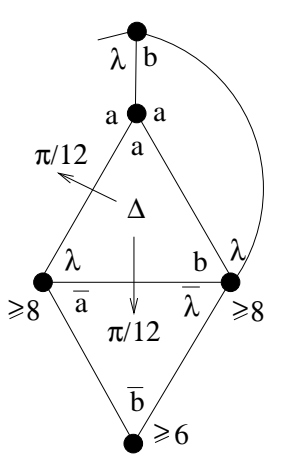

(i)

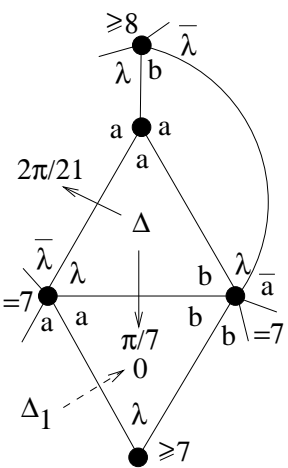

(vi)

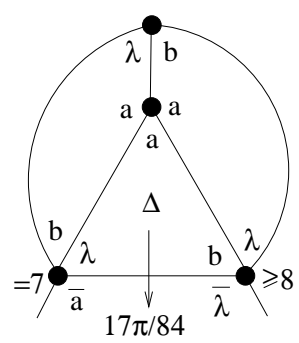

(x)

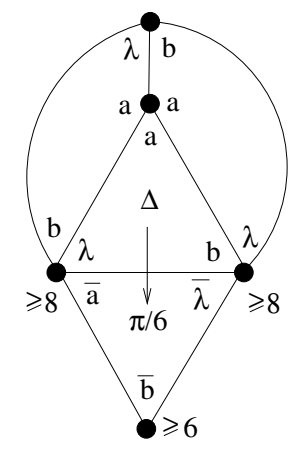

(xiv)

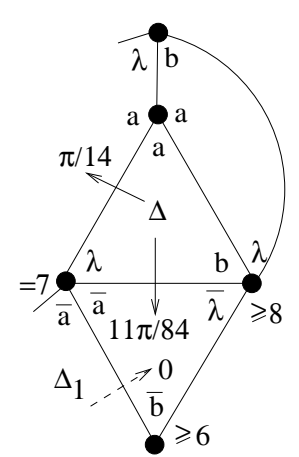

(ii)

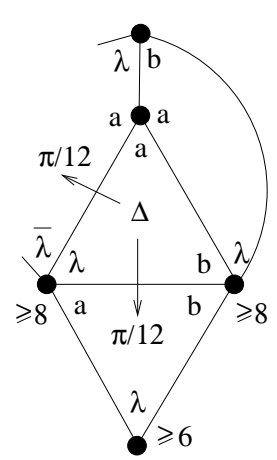

(iii)

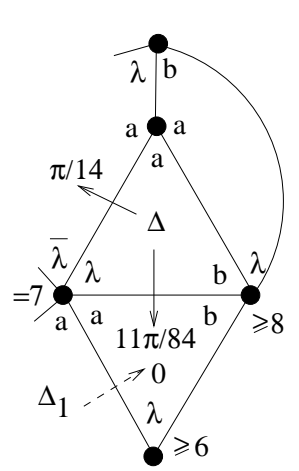

(iv)

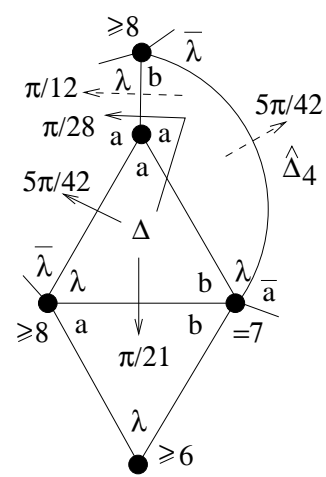

(v)
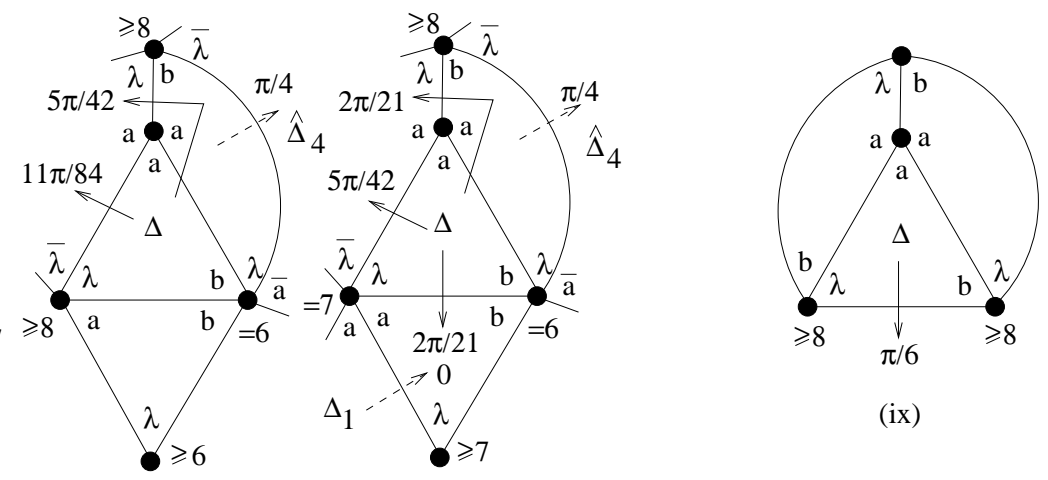

(ix)

(viii)

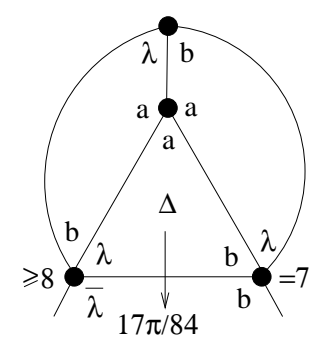

(xi)

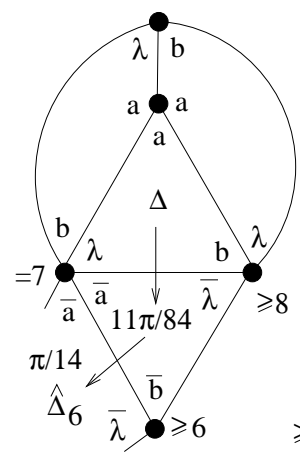

(xv)

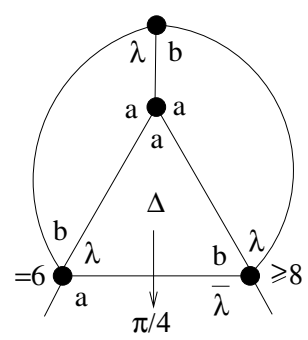

(xii)

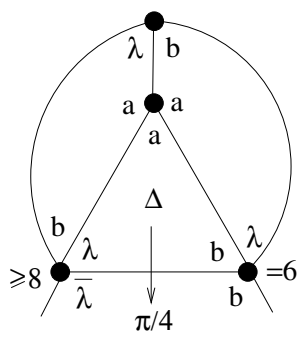

(xiii)

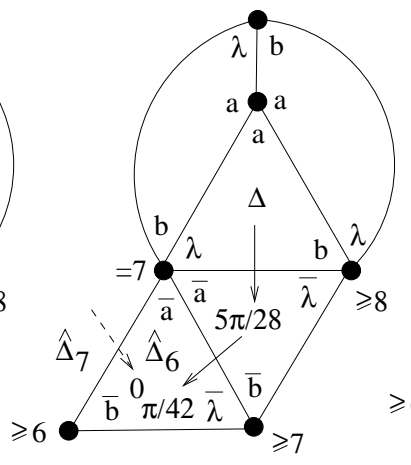

(xvi)
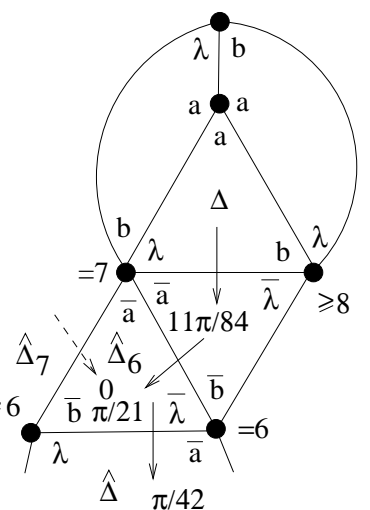

(xvii)

Figure 3.3: distribution of curvature for $n=9$ 


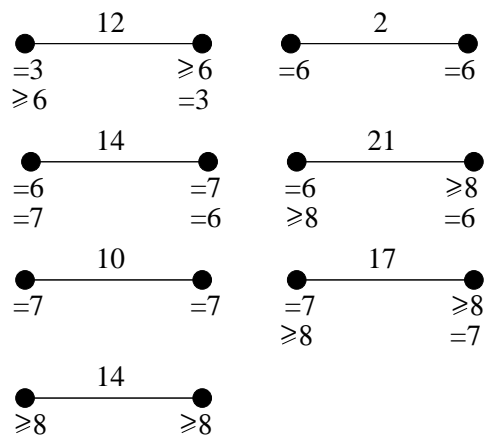

(i)
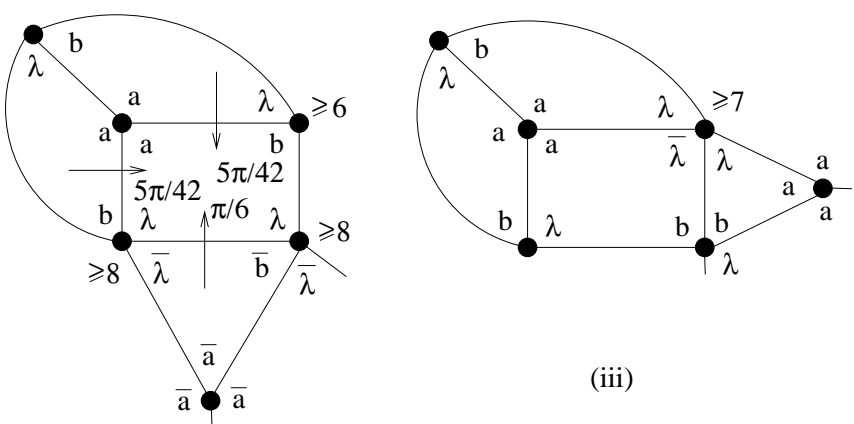

(iii)

(ii)

Figure 3.4: maximum curvature across a given edge and $d(\hat{\Delta})=4$ for $n=9$

$d\left(\hat{\Delta}_{i}\right)=3(1 \leq i \leq 3)$ : Figure 3.3(xiv)-(xvii). Distribute $c(\Delta)$ according to $d\left(v_{3}\right)$ and $d\left(\hat{\Delta}_{6}\right)$. Note that in Figure 3.3(xv) $c(\Delta) \leq \frac{17 \pi}{84}$ is added to $c\left(\hat{\Delta}_{2}\right) \leq-\frac{11 \pi}{84}$ and the difference $\frac{\pi}{14}$ is distributed further to $\hat{\Delta}_{6}$ under the assumption $d\left(\hat{\Delta}_{6}\right)>3$; in (xvi) $c(\Delta) \leq \frac{17 \pi}{84}$ is added to $c\left(\hat{\Delta}_{2}\right) \leq-\frac{5 \pi}{28}$ and the difference $\frac{\pi}{42}$ is distributed further to $\hat{\Delta}_{6}$; and in (xvii) $c(\Delta) \leq \frac{17 \pi}{84}$ is added to $c\left(\hat{\Delta}_{2}\right) \leq-\frac{11 \pi}{84}$, the difference $\frac{\pi}{84}$ is added to $c\left(\hat{\Delta}_{6}\right) \leq-\frac{\pi}{21}$ and then that difference $\frac{\pi}{42}$ is distributed further to $\hat{\Delta}$. Note that $l \neq k$ forces the corner label $\lambda$ in $\hat{\Delta}$ and so $d(\hat{\Delta})>3$.

Given the above distribution scheme, in Figure 3.4(i) the maximum amount that is distributed across an edge according to the degree of its endpoints is shown in multiples of $\frac{\pi}{84}$. In particular, the maximum amount of curvature sent across an edge is $\frac{\pi}{4}$. Indeed the values $12,14,10,14,2,21,17$ are given by Figure 3.2(xv), 3.2(vii), 3.2(ix), 3.3(ix), 3.3(xvii), 3.3(vii), (viii), (xii) and (xiii), $3.3(\mathrm{x})$ and $(\mathrm{xi})$ respectively.

Let $\hat{\Delta}$ receive positive curvature. If $d(\hat{\Delta})=q \geq 6$ then $c^{*}(\hat{\Delta}) \leq c(3,6,6, \ldots, 6)+q\left(\frac{\pi}{4}\right)<0$; if $d(\hat{\Delta})=5$ then either $c^{*}(\hat{\Delta}) \leq c(3,6,6,6,6)+2\left(\frac{\pi}{7}\right)+3\left(\frac{\pi}{42}\right)<0$ (see Figure 3.4(i)), or $c^{*}(\hat{\Delta}) \leq c(3,6,6,6,7)+2\left(\frac{\pi}{7}\right)+3\left(\frac{\pi}{4}\right)<0$, or $c^{*}(\hat{\Delta}) \leq c(6,6,6,6,6)+5\left(\frac{\pi}{4}\right)<0$.

Let $d(\hat{\Delta})=4$. Then $\hat{\Delta}$ is given by Figure 2.1(v) or (vi). Checking Figures 3.2 and 3.3 shows that curvature is never transferred across a $(b, \lambda)$-edge, in particular, $\hat{\Delta}$ does not receive across the $\left(u_{2}, u_{3}\right)$-edge in Figure 2.1(v) or the $\left(u_{3}, u_{4}\right)$-edge in Figure 2.1(vi). Suppose that $d\left(u_{1}\right) \neq 3$ in $\hat{\Delta}$. Then, using Figure 3.4(i), either $c^{*}(\hat{\Delta}) \leq c(6,6,6,6)+3\left(\frac{\pi}{42}\right)<0$; or $c^{*}(\hat{\Delta}) \leq c(6,6,6,7)+$ $2\left(\frac{\pi}{4}\right)+\frac{\pi}{42}<0$; or $c^{*}(\hat{\Delta}) \leq c(6,6,7,7)+3\left(\frac{\pi}{4}\right)<0$. Suppose now that $d\left(u_{1}\right)=3$. Then checking Figures 3.2 and 3.3 shows that the maximum total combined curvature $\hat{\Delta}$ of Figure $2.1(\mathrm{v})$, (vi) can receive across its $\left(u_{1}, u_{2}\right)$ and $\left(u_{4}, u_{1}\right)$ edges is $2\left(\frac{5 \pi}{42}\right), \frac{11 \pi}{84}+\frac{10 \pi}{84}$ respectively and so $c^{*}(\hat{\Delta}) \leq c(\hat{\Delta})+\frac{\pi}{4}+\frac{\pi}{4}$. Let $\hat{\Delta}$ receive across at most two edges. Then it is clear from Figure $3.4(\mathrm{i})$ that either there are no vertices of degree $\geq 8$ and so $c^{*}(\hat{\Delta}) \leq c(3,6,6,6)+2\left(\frac{14 \pi}{84}\right)=0$; or $c^{*}(\hat{\Delta}) \leq c(3,6,6,8)+\frac{14 \pi}{84}+\frac{\pi}{4}=0$ since, given $l\left(u_{1}\right)=a^{3}$, the only way $\frac{14 \pi}{84}$ can be exceeded is shown in Figure 3.3(xi) or (xiii). Now assume that $\hat{\Delta}$ receives across three edges. If $\hat{\Delta}$ is given by Figure 2.1(v) then, since no curvature is distributed from an $(a, b)$-edge of $\Delta$ across a $(\lambda, \lambda)$-edge of $\hat{\Delta}$, the only possibility is shown in Figure 3.4(ii) and $c^{*}(\hat{\Delta}) \leq c(3,6,8,8)+2\left(\frac{5 \pi}{42}\right)+\frac{\pi}{6}<0$. Let $\hat{\Delta}$ be given by Figure 2.1(vi). Then $\hat{\Delta}$ is shown in Figure 3.4(iii) in which $d\left(u_{2}\right) \geq 7$ and $\left(d\left(u_{3}\right), d\left(u_{4}\right)\right) \in\{(6, \geq 8),(7, \geq 8),(\geq 8,6),(\geq 8,7),(\geq 8, \geq 8)\}$. Since $c(3,7,7,8)=-\frac{43 \pi}{84}$ and $c(3,6,8,8)=-\frac{\pi}{2}$ it remains to consider $d\left(u_{2}\right)=7, d\left(u_{3}\right)=6$ and $d\left(u_{4}\right) \geq 8$ or $d\left(u_{2}\right)=7$, $d\left(u_{3}\right) \geq 8$ and $d\left(u_{4}\right)=6$. But then $\hat{\Delta}$ is not $\hat{\Delta}_{2}$ of Figure $3.3\left(\right.$ xi) or (xiii) therefore $c^{*}(\hat{\Delta}) \leq$ 
$c(3,6,7,8)+\frac{11 \pi}{84}+\frac{5 \pi}{42}+\frac{14 \pi}{84}<0$.

Finally let $d(\hat{\Delta})=3$. Using the fact that $c(6,6,7)=-\frac{\pi}{21}, c(6,7,7)=-\frac{2 \pi}{21}, c(6,7,8)=-\frac{11 \pi}{84}$, $c(6,8,8)=-\frac{\pi}{6}, c(7,7,7)=-\frac{\pi}{7}$ and $c(7,7,8)=-\frac{5 \pi}{28}$ we see from Figures 3.2 and 3.3 that if $\hat{\Delta}$ receives curvature across exactly one edge then $c^{*}(\hat{\Delta}) \leq 0$. Again an inspection of Figures 3.2 and 3.3 shows that $\hat{\Delta}$ does not receive across a $(b, \lambda)$-edge and that $\hat{\Delta}$ does not receive from $\Delta_{1}$ in Figure 3.2(xviii), 3.3(ii), 3.3(iv), 3.3(vi) or 3.3(viii); and that $\hat{\Delta}_{6}$ does not receive from $\hat{\Delta}_{7}$ in Figure 3.3(xvi) or (xvii). It follows that if $\hat{\Delta}$ receives curvature across two edges then the $(\lambda, a)$-edge is given by Figure 3.2 (xvii) or 3.3(i) or 3.3(xiv) and the $(a, b)$-edge is given by Figure $3.3\left(\right.$ iii) or $(\mathrm{v})$. But then $c^{*}(\hat{\Delta}) \leq c(8,8,8)+\frac{\pi}{6}+\frac{\pi}{12}=0$; or $c^{*}(\hat{\Delta}) \leq c(7,8,8)+\frac{\pi}{6}+\frac{\pi}{21}=0$.

\section{$3.3 n=8(l \neq k, l \neq 2 k, k \neq 2 l)$}

If $d(v) \leq 7$ then $l(v) \in\left\{b^{4}, a^{2} \lambda b \lambda^{-1}, b^{4} \lambda^{-1} \lambda, a^{2} \lambda \lambda^{-1} \lambda b \lambda^{-1}, a^{2} \lambda b \lambda^{-1} \lambda \lambda^{-1}, a^{2} \lambda b^{-3} \lambda^{-1}\right\}$ by Lemma 2.2. Therefore if $l(v)$ involves $a$ then either $d(v)=5$ or $d(v) \geq 7$; and if $l(v)$ involves b then either $d(v)=4$ or $d(v) \geq 6$. Much use will be made of the assumptions $l \neq k, l \neq 2 k$, $k \neq 2 l$ often without explicit mention.

If $c(\Delta)>0$ then $\Delta$ is given by Figure 3.1(i). Assume first that $d\left(v_{2}\right) \neq 4$ in $\Delta$. If $d\left(\hat{\Delta}_{i}\right)>3$ $(1 \leq i \leq 3)$ then distribute $\frac{1}{3} c(\Delta)$ to each $c\left(\hat{\Delta}_{i}\right)$; or if exactly one of the $\hat{\Delta}_{i}$ has degree 3 then distribute $\frac{1}{2} c(\Delta)$ to each of the other two regions; or if exactly two of the $\hat{\Delta}_{i}$ have degree 3 then distribute $c(\Delta)$ to the third region. Given this, in Figure 3.5(i) the maximum amount of curvature in multiples of $\frac{\pi}{420}$ crossing an edge according to the degree of its endpoints is indicated.

Convention. In this subsection and in subsection 3.4, for ease of presentation and understanding, we will say that the curvature equals $\alpha$ to mean that the curvature is $\alpha \pi / 420$. For the benefit of the reader we give the following examples: $c(5,5,5)=84(=\pi / 5) ; c(5,5,6)=56$; $c(5,5,7)=36 ; c(5,5,8)=21 ; c(5,6,6)=28 ; c(5,6,7)=8 ; c(5,6,8)=-7 ; c(5,7,7)=-12 ;$ $c(5,7,8)=-27 ; c(4,5,6)=98 ; c(4,5,7)=78 ; c(4,5,8)=63 ; c(4,6,6)=70 ; c(4,6,7)=50 ;$ $c(4,6,8)=35 ; c(4,7,7)=30 ;$ and $c(4,7,8)=15$.

Now let $d\left(\hat{\Delta}_{i}\right)=3(1 \leq i \leq 3)$. If $\Delta$ and the $\hat{\Delta}_{i}$ are given by Figure 3.6 (i),(ii) or (iv) then $c(\Delta)<0$; if by (iii) then $l \neq k$ forces $d(v) \geq 6$ and so, as shown, add $c(\Delta)=8\left(=\frac{2 \pi}{105}\right)$ to $c\left(\hat{\Delta}_{2}\right) \leq c(6,6,7)=-20$; if by (v) in which we assume $d(v) \geq 6$ then add $c(\Delta)=8$ to $c\left(\hat{\Delta}_{2}\right) \leq-20$; or if by (vi) in which $d(v)=5$ then add $c(\Delta)+c\left(\hat{\Delta}_{2}\right)=16$ to $c\left(\hat{\Delta}_{6}\right)$.

This leaves the case when $\Delta$ and the $\hat{\Delta}_{i}$ are given by Figure 3.6 (vii) in which we fix the vertices $v_{i}(4 \leq v \leq 6)$ and which requires more detailed analysis. We proceed according to $d\left(v_{i}\right)$ $(1 \leq i \leq 3)$.

Let $d\left(v_{1}\right)=d\left(v_{2}\right)=5$ and $d\left(v_{3}\right) \geq 7$ so that $c(\Delta) \leq 36$. If $\Delta$ and the $\hat{\Delta}_{i}(1 \leq i \leq 3)$ are given by Figure 3.6(viii) in which $d\left(v_{4}\right)=5$ then add $\frac{1}{2}\left(c(\bar{\Delta})+c\left(\hat{\Delta}_{1}\right)\right) \leq 60$ to each of $c\left(\hat{\Delta}_{4}\right)$ and $c\left(\hat{\Delta}_{5}\right)$; if by (ix) or (x) in which it is assumed that $d\left(\hat{\Delta}_{4}\right)>3$ then add $\frac{1}{2}\left(c(\Delta)+c\left(\hat{\Delta}_{1}\right)\right) \leq 46,36,57 / 2$ according to $d\left(v_{4}\right)=6,=7, \geq 8$ to $c\left(\hat{\Delta}_{4}\right)$ and to $c\left(\hat{\Delta}_{5}\right)$; or if by (xi) then add $c(\Delta)+c\left(\hat{\Delta}_{3}\right) \leq$ $72,24,9$ according to $d\left(v_{6}\right)=5,=7, \geq 8$ to $c\left(\hat{\Delta}_{B}\right)$.

Let $d\left(v_{1}\right)=d\left(v_{3}\right)=5$ and $d\left(v_{2}\right) \geq 7$. Assume firstly that $d\left(v_{4}\right)=5$. If $\Delta$ and the $\hat{\Delta}_{i}$ are given by Figure $3.7(\mathrm{i})$ in which $d\left(v_{2}\right)=7$ then add $\frac{1}{2}\left(c(\Delta)+c\left(\hat{\Delta}_{1}\right)\right)=36$ to $c\left(\hat{\Delta}_{4}\right)$ and $c\left(\hat{\Delta}_{5}\right)$; or if by (ii) in which $d\left(v_{2}\right) \geq 8$ then add $\frac{1}{2}\left(c(\Delta)+c\left(\hat{\Delta}_{1}\right)\right) \leq 21$ to $c\left(\hat{\Delta}_{4}\right)$ and $c\left(\hat{\Delta}_{5}\right)$ if $d\left(\hat{\Delta}_{5}\right)>3$ otherwise add $c(\Delta)+c\left(\hat{\Delta}_{1}\right) \leq 42$ to $c\left(\hat{\Delta}_{4}\right)$. Now assume that $d\left(v_{4}\right) \geq 6$. If $d\left(\hat{\Delta}_{4}\right)>3$ as in Figure 3.7(iii) then add $c(\Delta)+c\left(\hat{\Delta}_{1}\right)=44,=24, \leq 9$ according to $d\left(v_{4}\right)=6,=7, \geq 8$ to $c\left(\hat{\Delta}_{4}\right)$; or if $d\left(\hat{\Delta}_{4}\right)=3$ and $d\left(\hat{\Delta}_{8}\right)>3$ as in (iv) then add $\frac{1}{2}\left(c(\Delta)+c\left(\hat{\Delta}_{3}\right)\right)=60,=36, \leq 57 / 2$ 


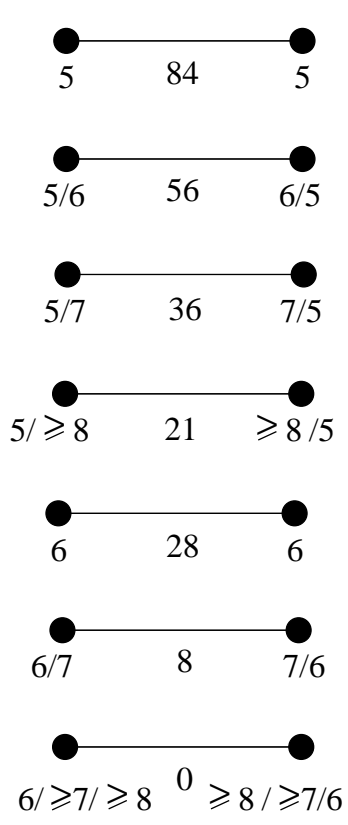

(i)
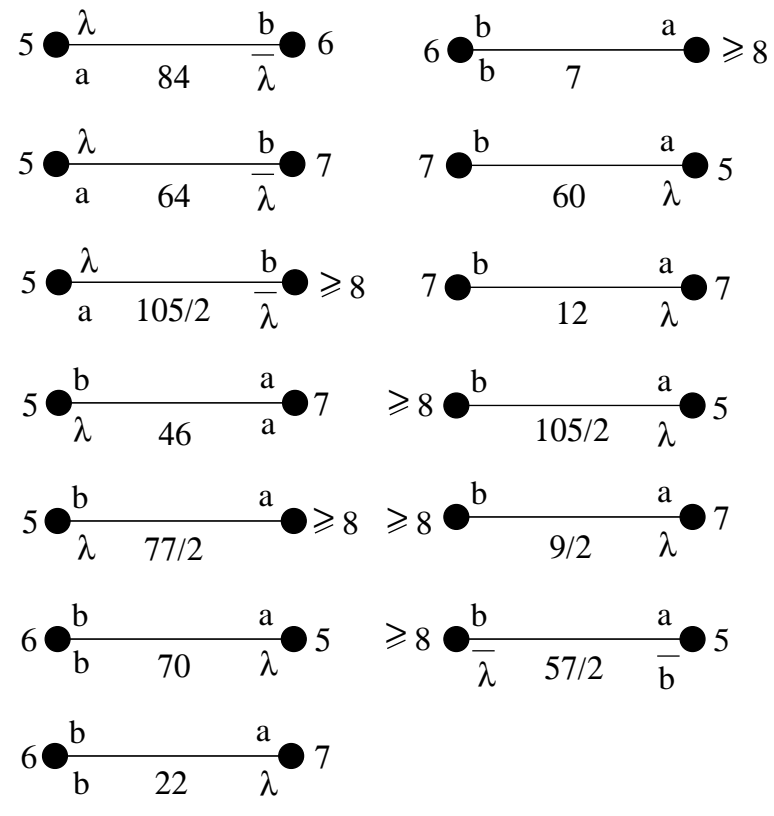

(ii)

Figure 3.5: maximum curvature across a given edge for $n=8$

according to $d\left(v_{6}\right)=5,=7, \geq 8$ to $c\left(\hat{\Delta}_{8}\right)$ and $c\left(\hat{\Delta}_{9}\right)$; or if $d\left(\hat{\Delta}_{4}\right)=d\left(\hat{\Delta}_{8}\right)=3$ as in (v) then add $c(\Delta)+c\left(\hat{\Delta}_{2}\right)=72,=44,=24, \leq 9$ according to $d\left(v_{5}\right)=5,=6,=7, \geq 8$ to $\hat{\Delta}_{A}$.

Let $d\left(v_{1}\right) \geq 7$ and $d\left(v_{2}\right)=d\left(v_{3}\right)=5$. Suppose that $d\left(v_{1}\right)=7$. If $\Delta$ and the $\hat{\Delta}_{i}$ are given by Figure 3.7 (vi) in which $d\left(v_{4}\right)=5$ then add $\frac{1}{2}\left(c(\Delta)+c\left(\hat{\Delta}_{1}\right)\right)=36$ to $\hat{\Delta}_{4}$ and $\hat{\Delta}_{5}$; if by

(vii) in which $d\left(v_{4}\right)=6$ then either add $\frac{1}{2}\left(c(\Delta)+c\left(\hat{\Delta}_{1}\right)\right)=22$ to each of $\hat{\Delta}_{4}$ and $\hat{\Delta}_{5}$ when $d\left(\hat{\Delta}_{4}\right)>3$ or add $c(\Delta)+c\left(\hat{\Delta}_{1}\right)=44$ to $\hat{\Delta}_{5}$; if by (viii) then either add $\frac{1}{2}\left(c(\Delta)+c\left(\hat{\Delta}_{1}\right)\right)=12, \leq 9 / 2$ to $\hat{\Delta}_{4}$ and $\hat{\Delta}_{5}$ when $d\left(\hat{\Delta}_{4}\right)>3$ or add $c(\Delta)+c\left(\hat{\Delta}_{1}\right)=24, \leq 9$ to $\hat{\Delta}_{5}$ when $d\left(\hat{\Delta}_{4}\right)=3$ according to $d\left(v_{4}\right)=7, \geq 8$.

Now suppose that $d\left(v_{1}\right) \geq 8$. If $\Delta$ and the $\hat{\Delta}_{i}$ are given by Figure $3.7(\mathrm{ix})$ in which $d\left(v_{4}\right)=5$ or 6 then either add $\frac{1}{2}\left(c(\Delta)+c\left(\hat{\Delta}_{1}\right)\right)=21$ or 7 to $\hat{\Delta}_{4}$ and $\hat{\Delta}_{5}$ when $d\left(\hat{\Delta}_{4}\right)=3$ otherwise add $c(\Delta)+c\left(\hat{\Delta}_{1}\right)=42$ or 14 to $\hat{\Delta}_{5}$; or if by (x) in which $d\left(v_{4}\right) \geq 7$ then add $c(\Delta) \leq 21$ to $c\left(\hat{\Delta}_{1}\right) \leq-27$.

Let $d\left(v_{i}\right)=5(1 \leq i \leq 3)$. If $\Delta$ and the $\hat{\Delta}_{i}$ are given by Figure 3.7(xi) in which $d\left(v_{4}\right)=5$ then add $\frac{1}{2}\left(c(\Delta)+c\left(\hat{\Delta}_{1}\right)\right)=84$ to $\hat{\Delta}_{4}$ and $\hat{\Delta}_{5}$; or if by Fgure 3.7(xii) or Figure 3.8(i) in which we assume $d\left(\hat{\Delta}_{4}\right)>3$ then add $\frac{1}{2}\left(c(\Delta)+c\left(\hat{\Delta}_{1}\right)\right)=70,=60, \leq 105 / 2$ to $\hat{\Delta}_{4}$ and $\hat{\Delta}_{5}$ according to $d\left(v_{4}\right)=6,=7, \geq 8$. Now let $d\left(\hat{\Delta}_{4}\right)=3$. Then add $\left.\frac{1}{3} c(\Delta)+c\left(\hat{\Delta}_{2}\right)\right)=84,=64, \leq 49$ as shown in Figure 3.8(ii). There remains $\frac{2}{3} c(\Delta)=56$ to be distributed. If $\Delta$ and the $\hat{\Delta}_{i}$ are given by Figures 3.8(iii),(iv) in which $d\left(\hat{\Delta}_{8}\right)>3$ then add $\frac{1}{2}\left(\frac{2}{3} c(\Delta)+c\left(\hat{\Delta}_{3}\right)\right)=70,=46, \leq 77 / 2$ to $c\left(\hat{\Delta}_{8}\right)$ and to $c\left(\hat{\Delta}_{B}\right)$ according to $d\left(v_{6}\right)=5,=7, \geq 8$; or if by (v) or (vi) in which $d\left(\hat{\Delta}_{8}\right)=3$ then add $\frac{1}{3} c(\Delta)+c\left(\hat{\Delta}_{3}\right)=112,=64, \leq 49$ to $c\left(\hat{\Delta}_{B}\right)$ according to $d\left(v_{6}\right)=5,=7, \geq 8$ and either add $\frac{1}{2}\left(\frac{1}{3} c(\Delta)+c\left(\hat{\Delta}_{2}\right)\right)=56,=32, \leq 49 / 2$ to each of $c\left(\hat{\Delta}_{6}\right)$ and $c\left(\hat{\Delta}_{A}\right)$ when $d\left(\hat{\Delta}_{6}\right)>3$ otherwise add $\frac{1}{3} c(\Delta)+c\left(\hat{\Delta}_{2}\right)=112,=64, \leq 49$ to $c\left(\hat{\Delta}_{A}\right)$ according to $d\left(v_{2}\right)=5,=7, \geq 8$. 


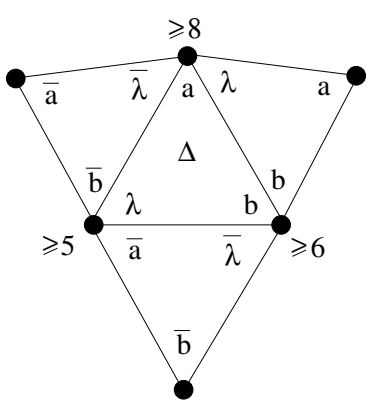

(i)

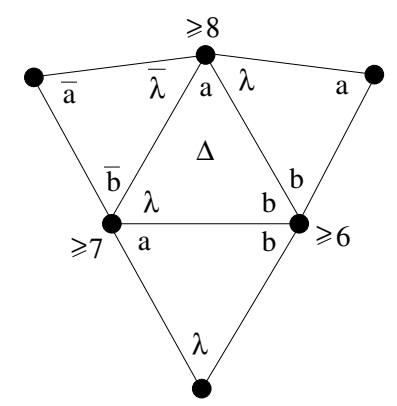

(iv)

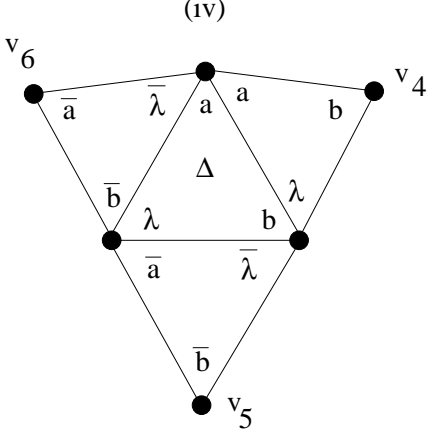

(vii)

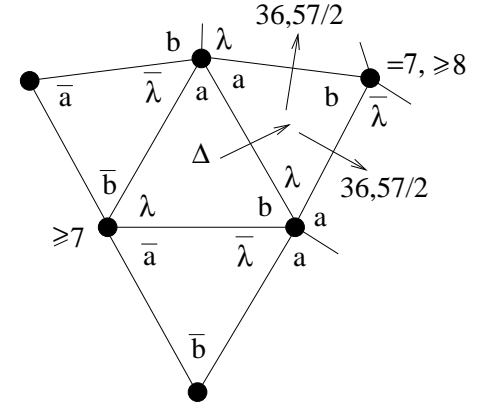

(x)

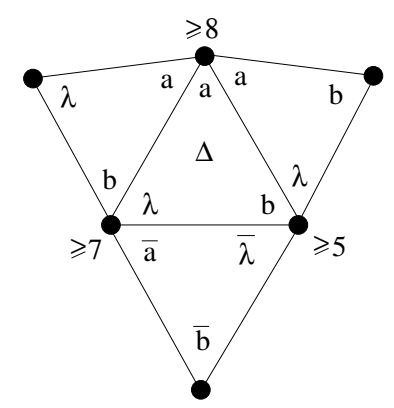

(ii)
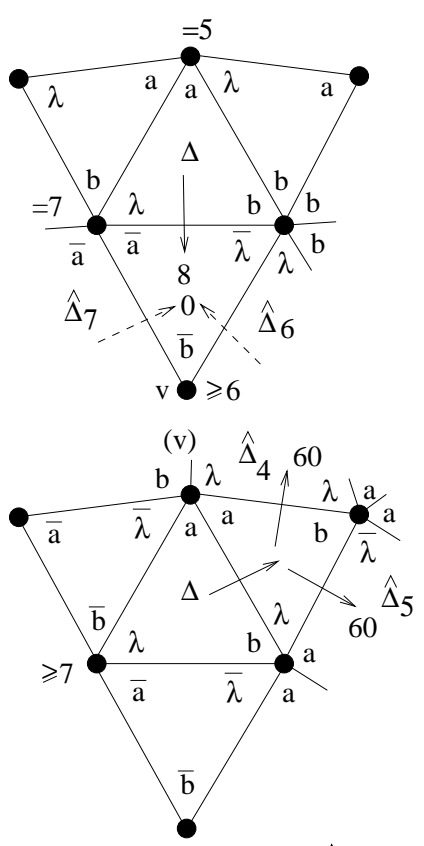

(viii) $\quad \hat{\Delta}_{\mathrm{B}} \quad \bar{\lambda} / \mathrm{a}$

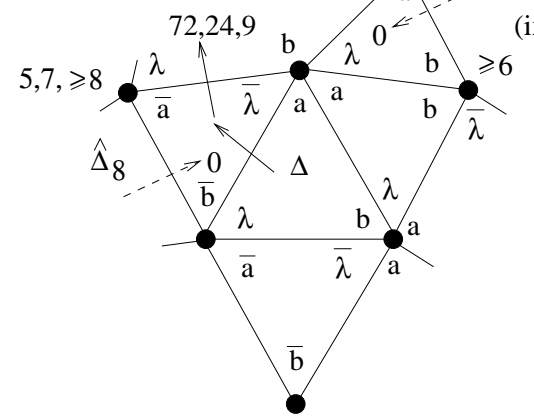

(xi)

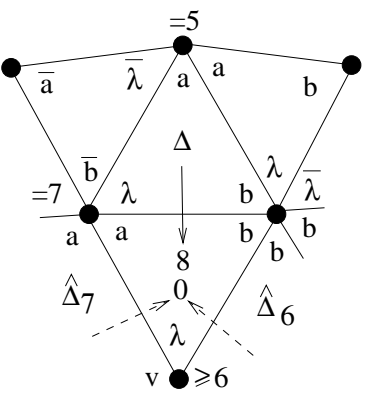

(iii)

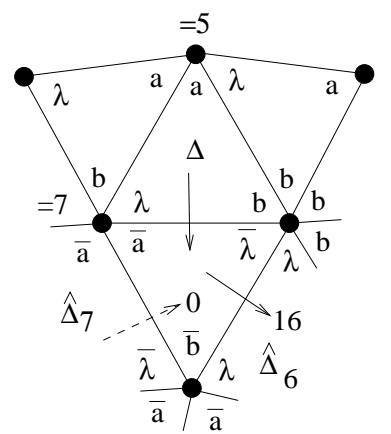

(vi)

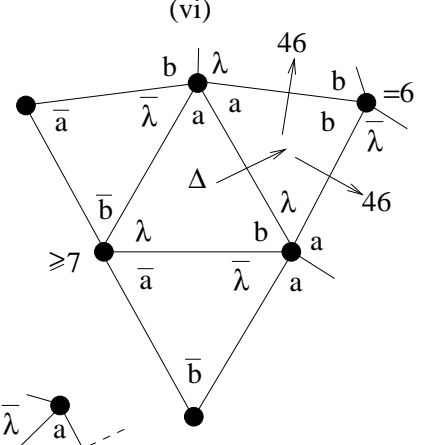

(ix)

Figure 3.6: curvature distribution for $n=8$ 


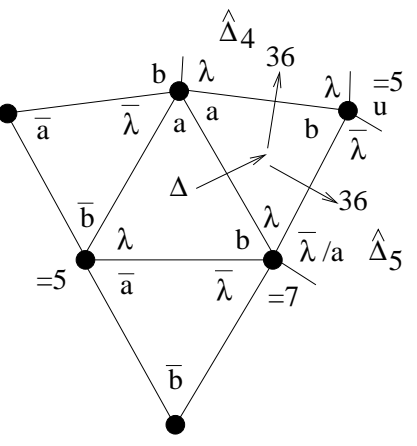

(i)

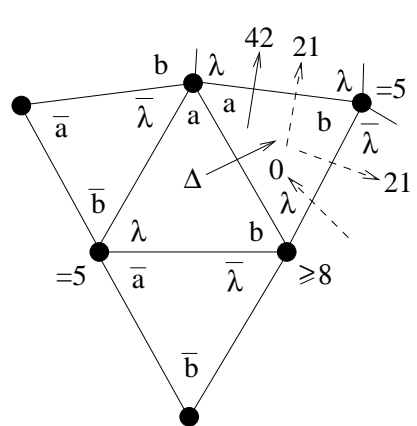

(ii)

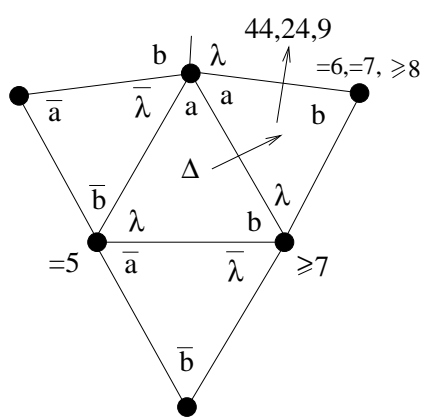

(iii)

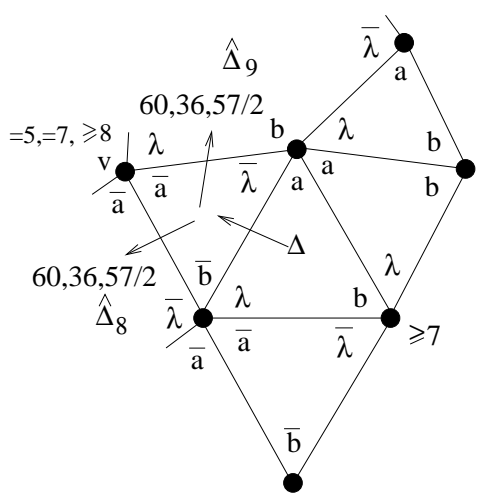

(iv)

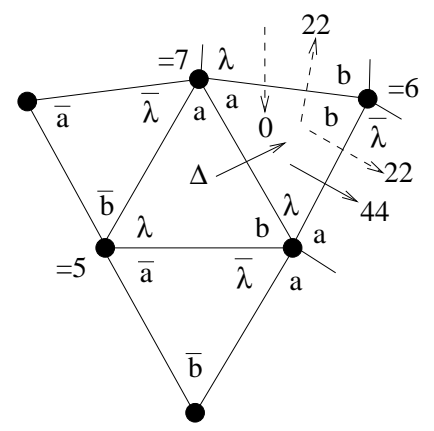

(vii)

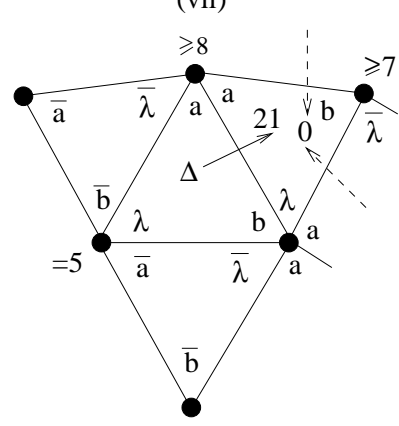

(x)

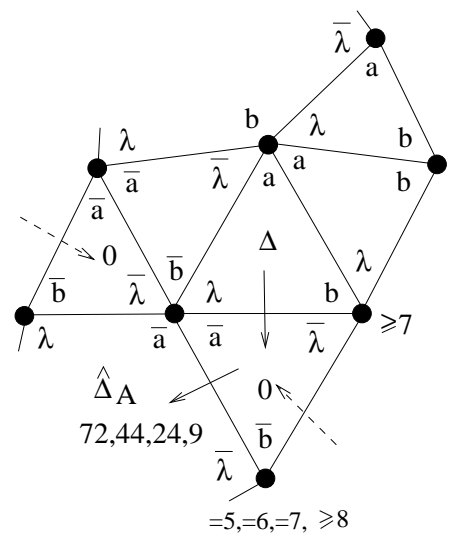

(v)

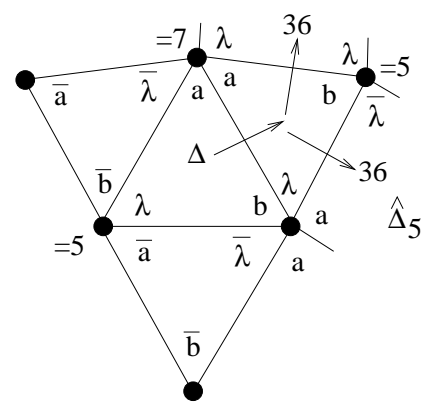

(vi)

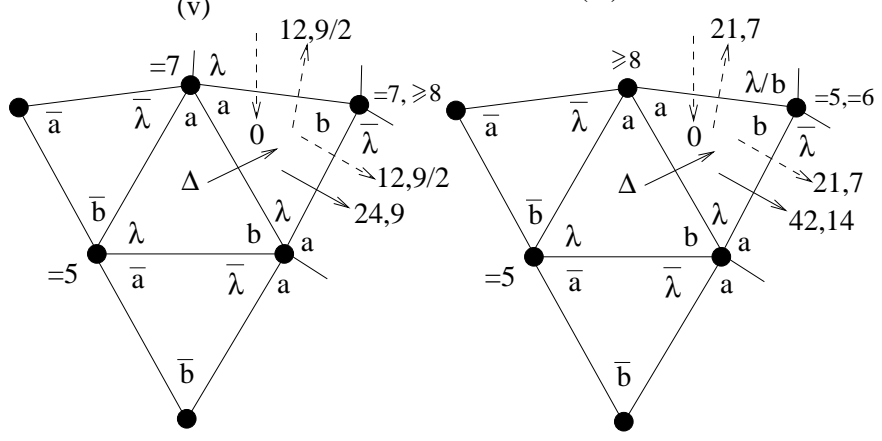

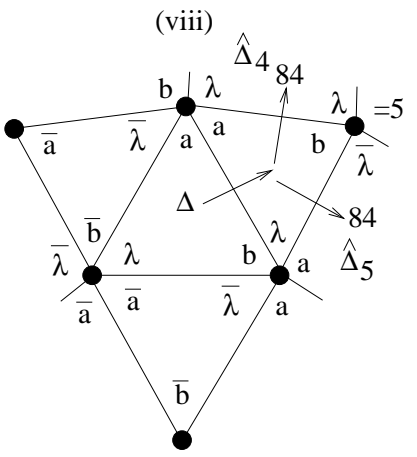

(xi)

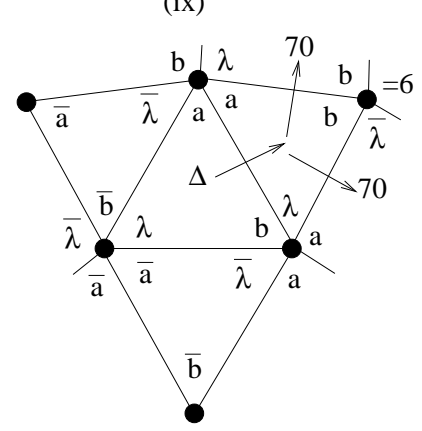

(xii)

Figure 3.7: curvature distribution for $n=8$ 


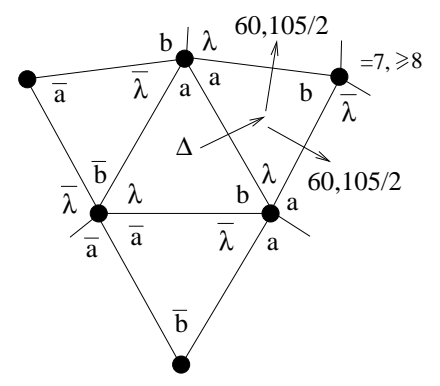

(i)

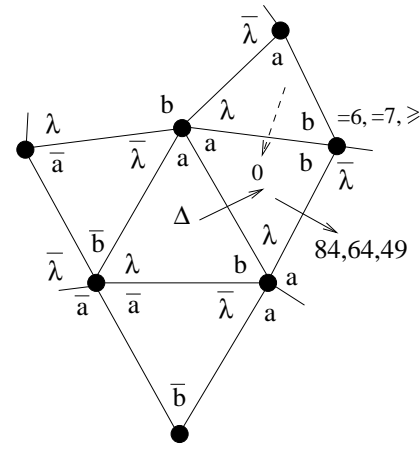

(ii)

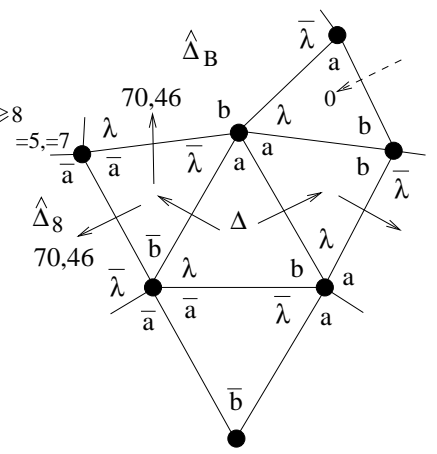

(iii)

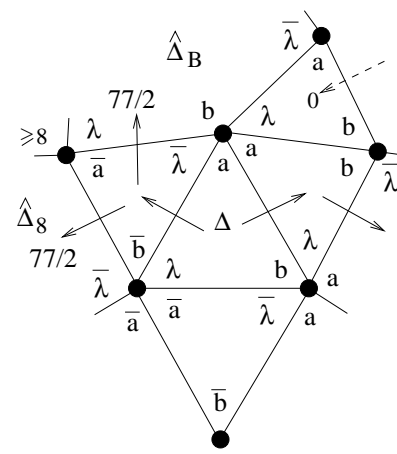

(iv)

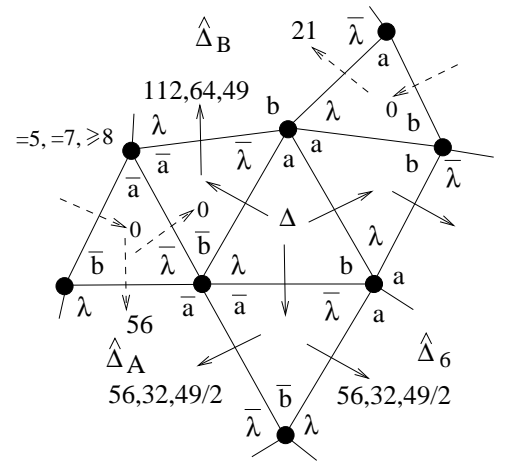

$=5,=7, \geqslant 8$

(v)

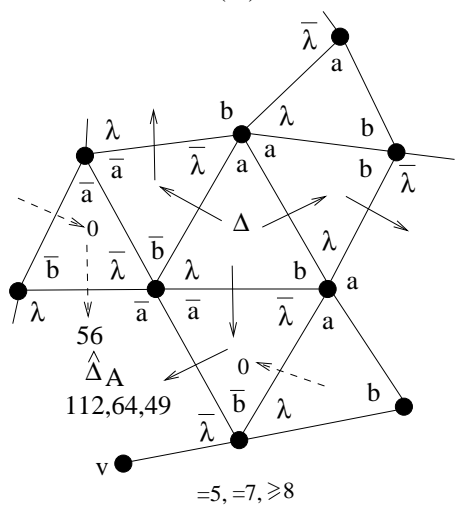

(vi)

Figure 3.8: curvature distribution for $n=8$ 


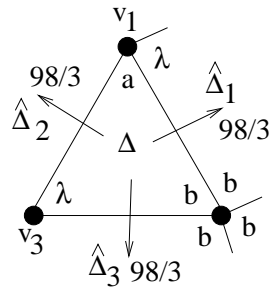

(i)

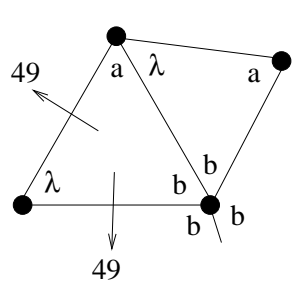

(ii)

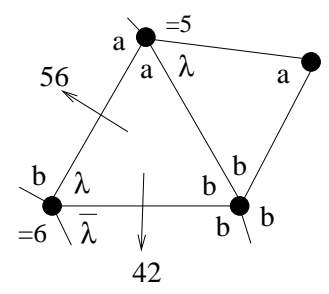

(iii)

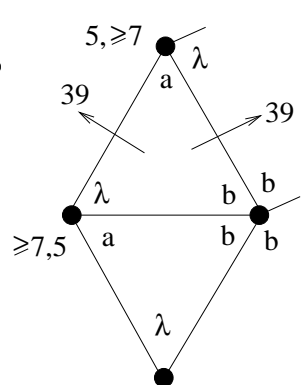

(iv)

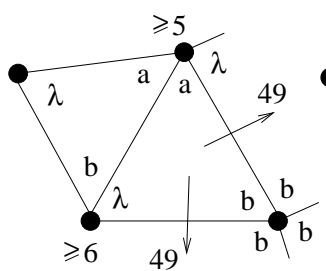

(v)

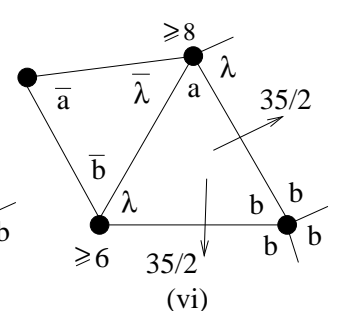

(vi)

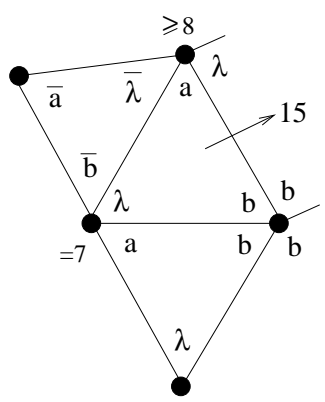

(vii)

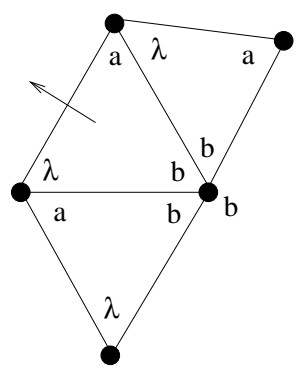

(viii)

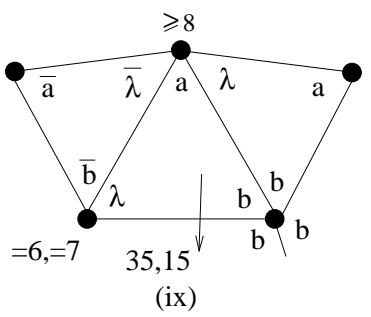

$\underbrace{}_{\lambda}$

(xii)

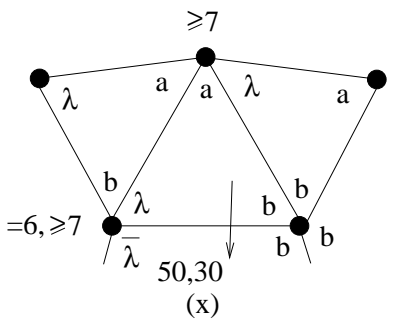

(x)

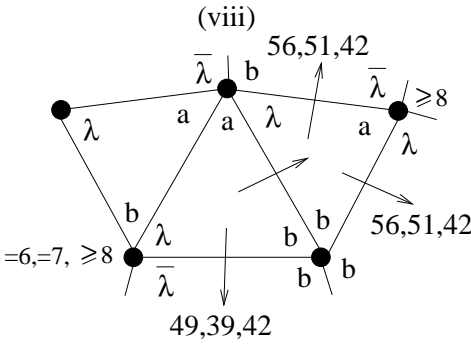

(xi)

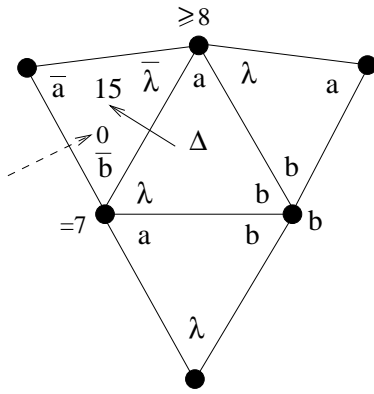

(xiii)

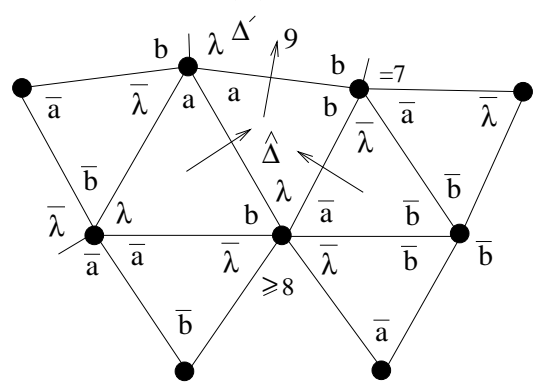

(xiv)

Figure 3.9: curvature distribution for $n=8$ when $d\left(v_{2}\right)=4$ 


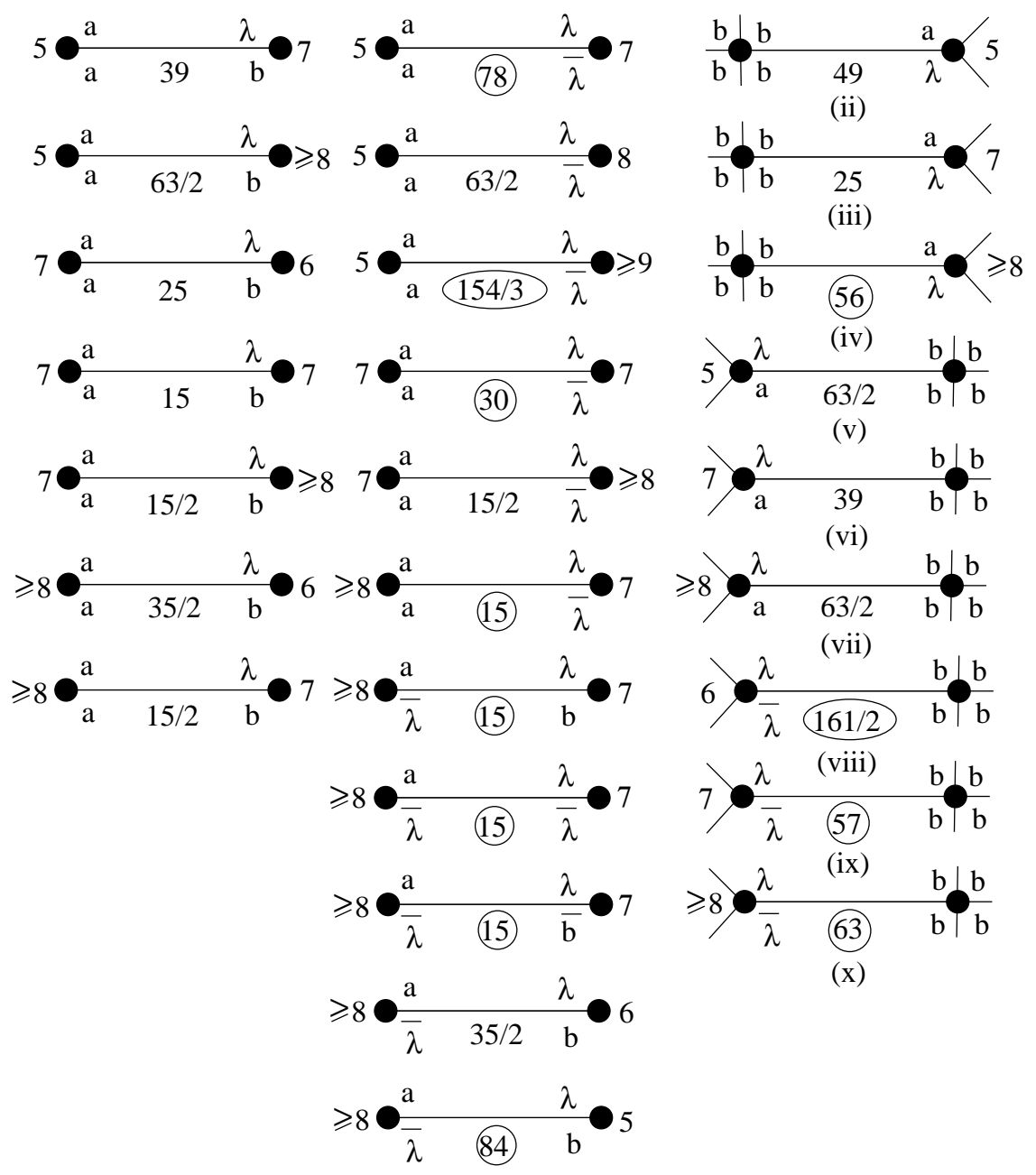

(i)

Figure 3.10: maximum curvature across an edge for $n=8$ 
This completes the distribution scheme for when $d\left(v_{2}\right) \neq 4$ in $\Delta$ and, given this, listed in Figure 3.5 (ii) are the 13 edges across which more curvature is transferred to $\hat{\Delta} \notin\left\{\hat{\Delta}_{A}, \hat{\Delta}_{B}\right\}$ than the corresponding edge in Figure 3.5(i) whose endpoints have the same valency. Indeed the values (in multiples of $\left.\frac{\pi}{420}\right) 84,64,105 / 2,46,77 / 2,70,22,7,60,12,105 / 2,9 / 2,57 / 2$ are obtained in Figure 3.8(ii),(ii),(i), (iii),(iv), 3.7(xii), (vii), (ix), 3.8(i), 3.7(viii), 3.8(i), 3.7(viii), 3.7(iv) respectively.

Now let $d\left(v_{2}\right)=4$ and so $\Delta$ is given by Figure 3.9(i) in which note that we cannot have $d\left(v_{1}\right)=d\left(v_{3}\right)=5$ so $c(\Delta) \leq c(4,5,6)=98$. If $d\left(\hat{\Delta}_{i}\right)>3(1 \leq i \leq 3)$ then distribute $\frac{1}{3} c(\Delta) \leq 98 / 3$ to each of $c\left(\hat{\Delta}_{i}\right)$ as shown; or if exactly one of the $\hat{\Delta}_{i}$ has degree 3 then $\frac{1}{2}(c(\Delta)$ is added to the other two as shown in Figures 3.9(ii)-(vi) apart from the one exception shown in Figure 3.9(iii) in which 42 of $c(\Delta)$ is added to $c\left(\hat{\Delta}_{2}\right)$ and the remaining 56 to $c\left(\hat{\Delta}_{3}\right)$. Suppose that exactly two of the $\hat{\Delta}_{i}$ have degree 3 . If $d\left(\hat{\Delta}_{2}\right)=d\left(\hat{\Delta}_{3}\right)=3$ then add $c(\Delta) \leq c(4,7,8)=15$ to $c\left(\hat{\Delta}_{1}\right)$ as shown in Figure 3.9 (vii). If $d\left(\hat{\Delta}_{1}\right)=d\left(\hat{\Delta}_{2}\right)=3$ then $\Delta$ is given by Figure 3.9 (viii) in which we note that if $d\left(v_{1}\right)=5$ or 7 then $l\left(v_{3}\right)=a \lambda \lambda^{-1} w$ which forces $d\left(v_{3}\right) \geq 9$. Add $c(\Delta) \leq 78,154 / 3,30,70 / 21,15,63$ according to $\left(d\left(v_{1}\right), d\left(v_{3}\right)\right)=(5,7),(5, \geq 9),(7,7),(7, \geq 9),(\geq$ $8,7),(\geq 8,5)$ respectively. If $d\left(\hat{\Delta}_{1}\right)=d\left(\hat{\Delta}_{3}\right)=3$ and $\Delta$ is given by Figure 3.9(ix) then add $c(\Delta) \leq 35,15$ to $c\left(\hat{\Delta}_{2}\right)$ according to $d\left(v_{3}\right)=6, \geq 7$ as shown; or if $\Delta$ is given by $(\mathrm{x})$ in which $d\left(v_{1}\right) \geq 7$ then add $c(\Delta) \leq 50,30$ to $c\left(\hat{\Delta}_{2}\right)$ according to $d\left(v_{3}\right)=6, \geq 7$; or if $\Delta$ is given by (xi) in which it is assumed that $d\left(v_{1}\right)=5$ and $d\left(\Delta_{5}\right)>3$ then add $\frac{1}{2} c(\Delta)=42$ or 39 to $c\left(\hat{\Delta}_{2}\right)$ and add $\frac{1}{2}\left(\frac{1}{2} c(\Delta)+c\left(\hat{\Delta}_{1}\right)\right)=56$ or 51 to each of $c\left(\hat{\Delta}_{4}\right)$ and $c\left(\hat{\Delta}_{5}\right)$ according to $d\left(v_{3}\right)=6$ or 7 , whereas if $d\left(v_{3}\right) \geq 8$ then add $\frac{2}{3} c(\Delta) \leq 42$ to $c\left(\hat{\Delta}_{2}\right)$ and add $\frac{1}{2}\left(\frac{1}{3} c(\Delta)+c\left(\hat{\Delta}_{1}\right)\right)=42$ to $c\left(\hat{\Delta}_{4}\right)$ and to $c\left(\hat{\Delta}_{5}\right)$; or by (xii) in which $d\left(v_{1}\right)=5$ and $d\left(\hat{\Delta}_{5}\right)=3$, if $d\left(v_{3}\right)=6$ then $161 / 2$ of $c(\Delta)=98$ is added to $c\left(\hat{\Delta}_{2}\right)$ and the remainder of $c(\Delta)$ plus $c\left(\hat{\Delta}_{1}\right) \leq 35 / 2+63=161 / 2$ to $c\left(\hat{\Delta}_{4}\right)$ as shown, or if $d\left(v_{3}\right)=7$ then 57 of $c(\Delta)=78$ is added to $c\left(\hat{\Delta}_{2}\right)$ and the remainder of $c(\Delta)$ plus $c\left(\hat{\Delta}_{1}\right) \leq 21+63=84$ is added to $c\left(\hat{\Delta}_{4}\right)$; or if $d\left(v_{3}\right) \geq 8$ then add $c(\Delta) \leq 63$ to $c\left(\hat{\Delta}_{2}\right)$ and $c\left(\hat{\Delta}_{1}\right) \leq 63$ to $c\left(\hat{\Delta}_{4}\right)$. If $d\left(\hat{\Delta}_{i}\right)=3(1 \leq i \leq 3)$ then add $c(\Delta) \leq 15$ to $c\left(\hat{\Delta}_{3}\right) \leq c(5,7,8)=-27$ as shown in Figure 3.9(xiii).

This completes the distribution scheme for $n=8$. Checking Figures $3.6-3.9$ shows that if $d(\hat{\Delta})=3$ then $\hat{\Delta}$ receives positive curvature across at most one edge apart from the one case when $\hat{\Delta}$ is $\hat{\Delta}_{1}$ of Figure 3.7(iii) and is the inverse of $\hat{\Delta}_{3}$ of Figure 3.9(xiii). Then $\hat{\Delta}$ is shown in Figure 3.9 (xiv) and in this case add $c\left(\Delta_{1}\right)+c\left(\Delta_{2}\right)+c(\hat{\Delta}) \leq 21+15-27=9$ to $c\left(\Delta^{\prime}\right)$ as shown. Note that $d\left(\Delta^{\prime}\right)>3$ since $\Delta^{\prime}$ corresponds to $\hat{\Delta}_{4}$ in Figure 3.7(iii). Also observe from checking Figures 3.6 - 3.9 that if $d(\hat{\Delta})=3$ and $\hat{\Delta}$ receives curvature across it's $(a, b)$-edge then $\hat{\Delta}=\hat{\Delta}_{2}$ of Figure 3.6(iii) in which $d\left(v_{3}\right)=7$ and $d(v) \geq 6$. This will be used throughout what follows without explicit mention however it implies for example that $\hat{\Delta}_{4}$ receives no curvature across it's $(a, b)$-edge in Figures 3.6(xi), 3.8(iii)-(v); and the same holds for $\hat{\Delta}_{8}$ in Figure 3.8(v) and (vi).

Given the above distribution scheme, we record (in multiples of $\frac{\pi}{420}$ ) in Figure 3.10(i) the maxima obtained when the values of Figure 3.5(i) are exceeded; and in Figure 3.10(ii)-(x) the maxima when an endpoint has degree 4. In Figure 3.10(i) the ten uncircled values are equal to $\frac{1}{2} c\left(4, d\left(v_{1}\right), d\left(v_{3}\right)\right)$. The only way this can be exceeded is to use Figure 3.9 (viii) or (xii) and this results in the eight circled $78, \frac{154}{3}, 30,15,15,15,15$ and 84 . The first seven of which equal $c\left(4, d\left(v_{1}\right), d\left(v_{3}\right)\right)$ in Figure 3.9 (viii) and the 84 corresponds to the 84 added to $c\left(\hat{\Delta}_{4}\right)$ in Figure 3.9(xii). In Figure 3.10(ii),(iii),(iv) respectively $d\left(v_{3}\right) \geq 6, \geq 6, \geq 5$. It is clear from Figure 3.9 (vii) that the only way $\frac{1}{2} c\left(4, d\left(v_{1}\right), d\left(v_{3}\right)\right)$ can be exceeded is in Figure 3.9 (xi) and this gives the circled value 56 which corresponds to the 56 added to $c\left(\hat{\Delta}_{5}\right)$. In Figure $3.10(\mathrm{v})$,(vi), (vii) respectively, $d\left(v_{1}\right) \geq 8, \geq 5, \geq 5$ and the three maximum values $63 / 2,39,63 / 2$ are $\frac{1}{2} c\left(4, d\left(v_{1}\right), d\left(v_{3}\right)\right)$ since these cannot be exceeded using Figure 3.9(ix). The three values $161 / 2,57,63$ of Figure 3.10 (viii)-(x) corresponds to the $161 / 2,57,63$ added to $c\left(\hat{\Delta}_{2}\right)$ in Figure 3.9 (xi) and these cannot be exceeded using $\frac{1}{2} c\left(4, d\left(v_{1}\right), d\left(v_{3}\right)\right)$ or Figure 3.9(ix).

Let $\hat{\Delta}$ receive positive curvature. Then 84 across an edge of $\hat{\Delta}$ is exceeded only by the 112 in Figure 3.8(v) and (vi). But checking Figures 3.6-3.9 shows that $\hat{\Delta}_{4}$ contributes at most 21 to 


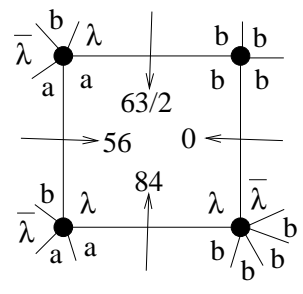

(i)

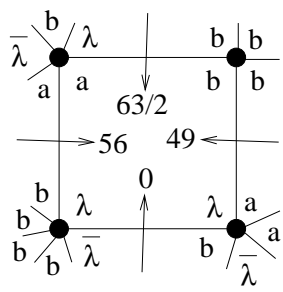

(ii)

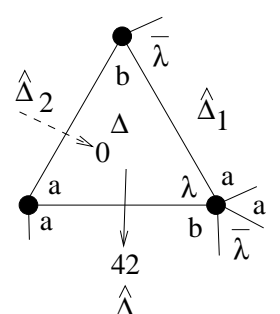

(iii)

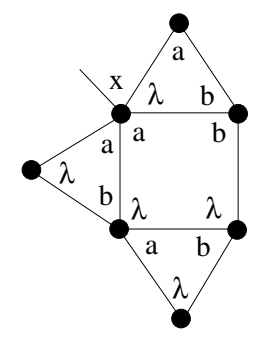

(iv)

Figure 3.11: curvature into regions of degree 4 for $n=8$

$\hat{\Delta}_{B}$ in Figure $3.8(\mathrm{v})$ and that $\hat{\Delta}_{8}$ contributes at most 56 to $\hat{\Delta}_{A}$ in Figure 3.8(v) and (vi). It follows that $c^{*}(\hat{\Delta}) \leq c(\hat{\Delta})+d(\hat{\Delta})(84)$ and so if $d(\hat{\Delta}) \geq 6$ then $c^{*}(\hat{\Delta}) \leq 0$. Let $d(\hat{\Delta})=5$. Since $c(5,5,5,5,5)+5(84)=0, c(4,5,5,6,6)+5(84)=-434+420<0$, and $c(4,5,5,5,7)+5(84)=$ $-426+420<0$ it remains to consider $c(4,5,5,5,5)=-378$ and $c(4,5,5,5,6)=-406$. From Figure 3.5(iv) we see that the maximum curvature across a $(4,5)$ - edge, $(4,6)$ - edge is $49,161 / 2$ respectively so it follows that $c^{*}(\hat{\Delta}) \leq c(4,5,5,5,5)+2(49)+3(84)<0$ or $c(4,5,5,5,6)+$ $49+161 / 2+3(84)<0$. Let $d(\hat{\Delta})=4$. If $\hat{\Delta}$ receive curvature across exactly one edge then $c^{*}(\hat{\Delta}) \leq c(4,5,5,5)+112=-126+112<0$.

Now assume that $\hat{\Delta}$ receives across more than one edge and $\hat{\Delta} \notin\left\{\hat{\Delta}_{A}, \hat{\Delta}_{B}\right\}$. Recall that $\hat{\Delta}$ is given by Figure 2.1(v) or (vi).

Suppose across exactly two edges. Since $c(5,5,5,5)+2(84)=0, c(4,5,6,6)+2(84)=-182+$ $168<0$ and $c(4,5,5,7)+2(84)=-174+168<0$ it remains to consider $c(4,5,5,5)$ and $c(4,5,5,6)=-156$. But, given $l \neq 2 k$ and $l \neq k$, any attempt at labelling implies that the only two possibilities are given by Figure 3.11(i) and (ii) in which the maximum $\hat{\Delta}$ can receive across an edge is indicated and so $c^{*}(\hat{\Delta}) \leq c(4,5,5,6)+56+84<0$.

We introduce the following notation: $d_{i}=d\left(u_{i}\right),(1 \leq i \leq 4)$ and $m_{i} \cdot\left(\frac{\pi}{420}\right)$ denotes an upper bound for the amount of curvature $\hat{\Delta}$ can receive across the $\left(u_{i}, u_{i+1}\right)$-edge $(1 \leq i \leq 4$, subscripts mod 4), in particular, $m_{i} \leq 84$. We write $\left(d_{1}, d_{2}, d_{3}, d_{4}\right)=\left(a_{1}, a_{2}, a_{3}, a_{4}\right)$ to mean $d_{i} \geq a_{i}$ and put $\Sigma=m_{1}+m_{2}+m_{3}+m_{4}$. Also define $\tilde{c}$ by $c\left(d_{1}, d_{2}, d_{3}, d_{4}\right)=-\tilde{c}\left(\frac{\pi}{420}\right)$, in particular if $\Sigma \leq \tilde{c}$ then $c^{*}(\hat{\Delta}) \leq 0$.

First let $\hat{\Delta}$ be given by Figure 2.1(v) and suppose that the four neighbouring regions of $\hat{\Delta}$ have degree 3. We see from Figure 3.12 that there are 24 possibilities. For 19 of these the value of $(\Sigma, \tilde{c})$ is straightforwardly obtained using: (i) the values in Figure 3.6; (ii) $m_{i}=84$ if this suffices to show $\Sigma \leq \tilde{c}$; (iii) $\hat{\Delta}$ receives at most 42 from $\Delta$ in Figure 3.11(iii) since $d\left(\hat{\Delta}_{1}\right)>3$ and $\Delta$ receives no curvature from $\hat{\Delta}_{2}$; (iv) $d\left(u_{1}\right) \geq 7$ in Figure 3.11(iv) since $l>k$ implies $x \neq b$.

The details are as follows: $(1,3,5,9),(5,6,5,7),(56,70,77 / 2,36),(401 / 2,244)$ by which is meant that four neighbouring regions are $\Delta_{1}, \Delta_{3}, \Delta_{5}$ and $\Delta_{9}$ which forces $\left(d_{1}, d_{2}, d_{3}, d_{4}\right)=$ $(5,6,5,7),\left(m_{1}, m_{2}, m_{3}, m_{4}\right)=(56,70,77 / 2,36)$ and $(\Sigma, \tilde{c})=(401 / 2,244) ;(1,3,6,8),(8,6,7,7)$, $(84,84,84,84),(336,355) ;(1,3,6,9),(5,6,7,5),(56,22,36,84),(198,244) ;(1,3,7,8),(8,6,7,6)$, $(84,22,84,84),(274,335) ;(1,3,7,9),(5,6,7,6),(56,22,8,84),(170,272) ;(1,4,5,8),(8,5,7,5)$, $(21,84,84,84),(273,279) ;(1,4,5,9),(5,5,7,7),(84,36,48,36),(240,264) ;(1,4,6,8),(8,5,7,7)$, $(21,84,84,84),(273,327) ;(1,4,7,8),(8,5,5,6),(21,84,56,84),(245,259) ;(2,3,5,9),(8,4,5,7)$, $(84,84,77 / 2,0),(412 / 2,237) ;(2,3,6,8),(5,4,7,7),(84,84,15,36),(219,222) ;(2,3,6,9),(8,4,7,5)$, $(84,84,36,21),(225,237) ;(2,3,7,9),(8,4,7,6),(84,84,8,0),(176,265) ;(2,4,5,8),(5,6,7,5)$, $(56,15,60,84),(215,244) ;(2,4,5,9),(8,6,7,7),(84,84,84,0),(252,355) ;(2,4,6,8),(5,6,7,7)$, $(56,84,15,84),(239,292) ;(2,4,6,9),(8,6,7,5),(84,84,84,21),(273,307) ;(2,4,7,8),(5,6,5,6)$, 


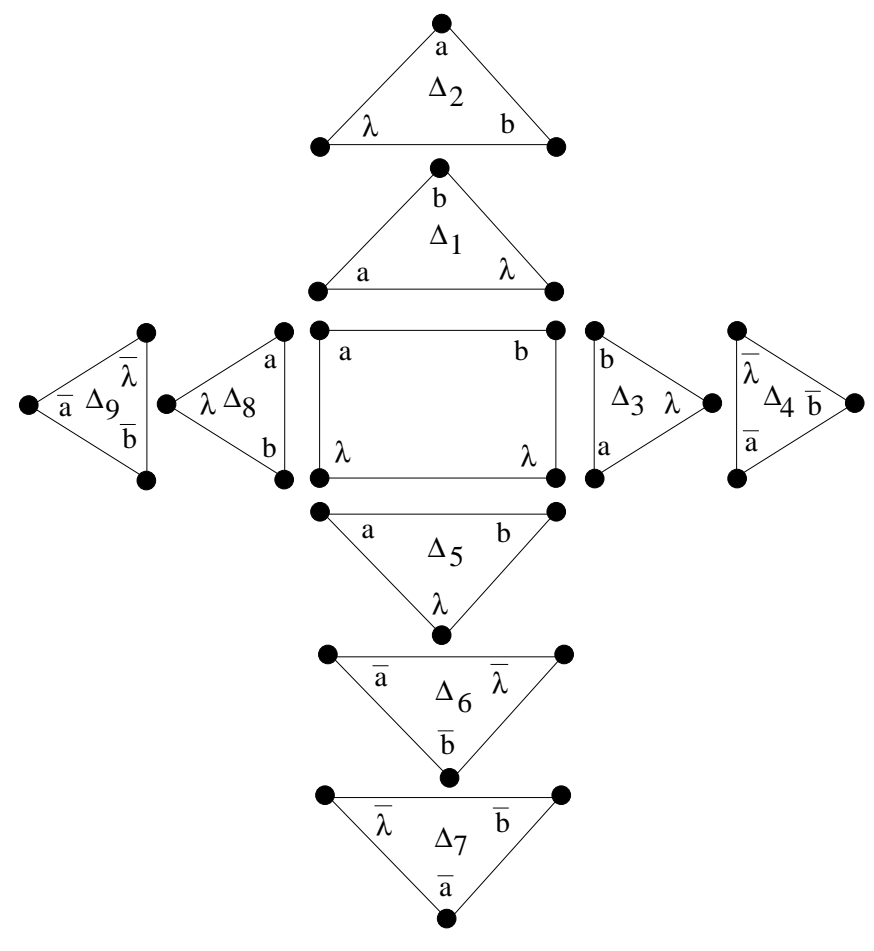

Figure 3.12: degree 3 neighbours of a degree 4 region

$(56,56,56,56),(244,244) ;(2,4,7,9),(8,6,5,6),(84,84,84,0),(252,287)$. The remaining five cases are dealt with in turn. If $(1,3,5,8)$ or $(2,3,5,8)$ then $l=2 k$, a contradiction. For $(1,4,6,9)$ we have $\left(d_{1}, d_{2}, d_{3}, d_{4}\right)=(5,5,7,5)$ and if $d_{1} \geq 7$ then $\left(m_{1}, m_{2}, m_{3}, m_{4}\right)=(36,84,36,84)$ and $(\Sigma, \tilde{c})=(240,264) ;$ or if $d_{1}=5$ but $d_{2} \geq 7$ then $\left(m_{1}, m_{2}, m_{3}, m_{4}\right)=(39,84,36,84)$ and $(\Sigma, \tilde{c})=(243,264)$; or if $d_{1}=d_{2}=5$ then (see Figure 3.11(iii)) $\left(m_{1}, m_{2}, m_{3}, m_{4}\right)=(42,36,36,84)$ and $(\Sigma, \tilde{c})=(198,216)$. For $(1,4,7,9)$ we have $\left(d_{1}, d_{2}, d_{3}, d_{4}\right)=(5,5,5,6)$ and if $d_{1} \geq 7$ then $\left(m_{1}, m_{2}, m_{3}, m_{4}\right)=(36,84,84,8)$ and $(\Sigma, \tilde{c})=(214,244)$; or if $d_{1}=5$ and $d_{2} \geq 7$ then $\left(m_{1}, m_{2}, m_{3}, m_{4}\right)=(39,36,84,56)$ and $(\Sigma, \tilde{c})=(215,244) ;$ or if $d_{1}=d_{2}=5$ and $d_{3} \geq 7$ then (see Figure 3.11(iii)) $\left(m_{1}, m_{2}, m_{3}, m_{4}\right)=(42,84,8,56)$ and $(\Sigma, \tilde{c})=(190,244)$; or if $d_{1}=d_{2}=d_{3}=5$ and $d_{4} \geq 7$ then $\left(m_{1}, m_{2}, m_{3}, m_{4}\right)=(42,84,36,36)$ and $(\Sigma, \tilde{c})=(198,216)$; or if $d_{1}=d_{2}=d_{3}=5$ and $d_{4}=6$ then this forces the third vertex of $\hat{\Delta}_{9}$ to have degree $\geq 8$ and so $\left(m_{1}, m_{2}, m_{3}, m_{4}\right)=(42,84,56,0)$ and $(\Sigma, \tilde{c})=(182,196)$. For $(2,3,7,8)$ we have $\left(d_{1}, d_{2}, d_{3}, d_{4}\right)=(5,4,7,6)$ and if $d_{2} \geq 6$ then $\left(m_{1}, m_{2}, m_{3}, m_{4}\right)=(84,84,8,56)$ and $(\Sigma, \tilde{c})=(232,272)$; or if $d_{2}=4$ then $\left(m_{1}, m_{2}, m_{3}, m_{4}\right)=(39,56,8,56)$ and $(\Sigma, \tilde{c})=(159,202)$.

Now suppose that exactly three of the neighbouring regions of $\hat{\Delta}$ are 3 -gons. Then (see Figure $3.12)$ there are 44 possibilities. For 35 cases the values of $(\Sigma, \tilde{c})$ again are straightforward and are as follows: $(1,3,6),(5,6,7,5),(84,22,84,-),(190,244) ;(1,3,7),(5,6,7,6),(84,22,84,-)$, $(190,244) ;(1,4,5),(5,5,7,5),(84,36,60,-),(180,216) ;(1,4,6),(5,5,7,5),(84,84,36,-),(204,216)$; $(2,3,6),(5,4,7,5),(56,56,36,-),(148,174) ;(2,3,7),(5,4,7,6),(84,84,8,-),(176,202) ;(2,4,5)$, $(5,6,7,5),(84,84,60,-),(228,244) ;(2,4,6),(5,6,7,5),(84,15,84,-),(183,244) ;(2,4,7),(5,6,5,6)$, $(84,84,56,-),(224,224) ;(1,3,8),(8,6,5,5),(84,84,-, 84),(252,259) ;(1,3,9),(5,6,5 / 7,6 / 5)$ (since labelling prevents $\left.d_{3}=d_{4}=5\right),(56,84,-, 84),(224,224) ;(1,4,8),(8,5,5,5),(21,84,-84)$, $(185,231) ;(2,3,9),(8,4,5,5),(84,84,-21),(189,189) ;(2,4,8),(5,6,5 / 7,6 / 5)$ (since labelling prevents $\left.d_{3}=d_{4}=5\right),(56,84,-84),(224,224) ;(2,4,9),(8,6,5,5),(84,84,-, 84),(252,259)$; $(1,5,8),(8,5,5,5),(84,-, 84,77 / 2),(413 / 2,231) ;(1,5,9),(5,5,5,7),(84,-, 77 / 2,36),(317 / 2,216)$; 


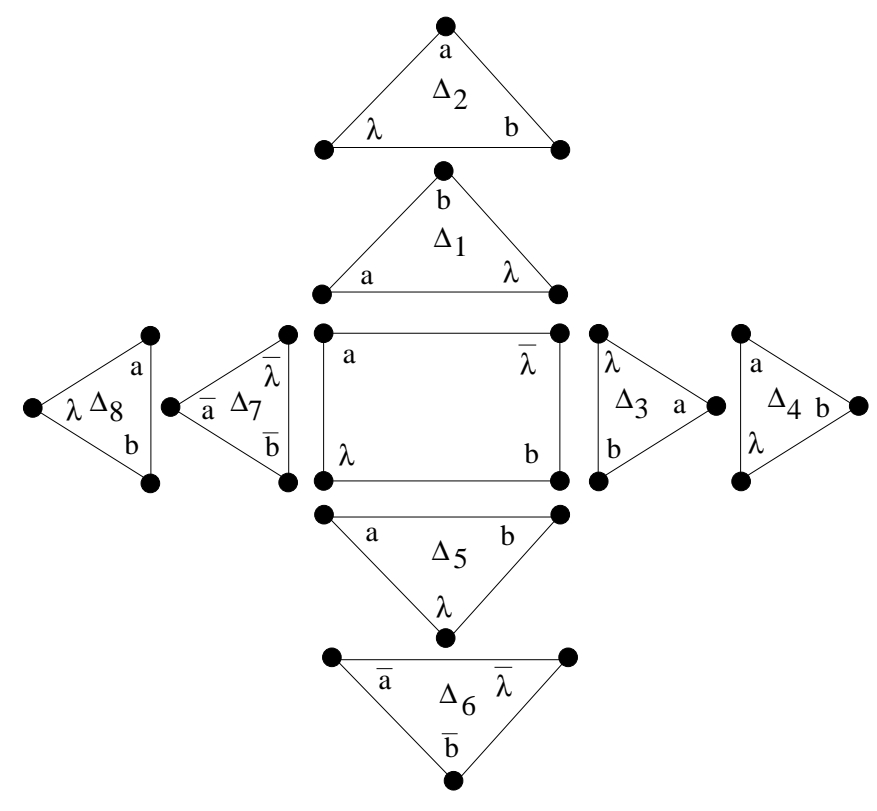

Figure 3.13: degree 3 neighbours of a degree 4 region

$(1,6,8),(8,5,6,7),(84,-, 84,84),(252,307) ;(1,7,8),(8,5,5,6),(84,-, 84,84),(252,259) ;(1,7,9)$, $(5,5,5,6),(84,-, 56,56),(196,196) ;(2,5,8),(7,4,5,5)$ (see Figure 3.11(iv)), $(39,-, 84,46)$, $(169,174) ;(2,5,9),(8,4,5,7),(84,-, 84,0),(168,237) ;(2,6,8),(5,4,6,7),(84,-, 15,36),(135,202)$; $(2,6,9),(8,4,6,5),(84,-, 84,21),(189,217) ;(2,7,9),(8,4,5,6),(84,-, 84,0),(168,217) ;(3,5,9)$, $(5,4,5,7),(-, 84,84,36),(164,174) ;(3,6,8),(5,4,7,7),(-, 84,15,84),(183,222) ;(3,7,8),(5,4,7,6)$, $(-, 84,8,84),(176,202) ;(3,7,9),(5,4,7,6),(-, 84,8,84),(176,202) ;(4,5,8),(5,5,7,5),(-, 36,60,84)$, $(180,216) ;(4,5,9),(5,5,7,7),(-84,84,84),(252,264) ;(4,6,8),(5,5,7,7),(-, 84,84,84),(252,264)$; $(4,6,9),(5,5,7,5),(-, 84,36,84),(204,216) ;(4,7,8),(5,5,5,6),(-, 84,56,56),(196,196) ;(4,7,9)$, $(5,5,5,6),(-, 84,56,56),(196,196)$.

There are nine cases remaining. For $(1,3,5)$ we have $\left(d_{1}, d_{2}, d_{3}, d_{4}\right)=(5,6,5,5)$ so if $d_{2}=6$ then $\left(m_{1}, m_{2}, m_{3}\right)=(56,56,84)$ and $(\Sigma, \tilde{c})=(196,196)$; or if $d_{2} \geq 7$ then $\left(m_{1}, m_{2}, m_{3}\right)=(56,60,84)$ and $(\Sigma, \tilde{c})=(200,216)$. For $(1,4,7)$ we have $\left(d_{1}, d_{2}, d_{3}, d_{4}\right)=(5,5,5,6)$ so if $d_{2} \geq 7$ then $\left(m_{1}, m_{2}, m_{3}\right)=(39,84,56)$ and $(\Sigma, \tilde{c})=(179,244)$; or if $d_{2}=5$ then (see Figure 3.11(iii)) $\left(m_{1}, m_{2}, m_{3}\right)=(42,84,56)$ and $(\Sigma, \tilde{c})=(182,196)$. For $(2,3,5), l \neq 2 k$ prevents $d_{1}=d_{2}=5$ so $\left(d_{1}, d_{2}, d_{3}, d_{4}\right)=(5 / 7,4,5,7 / 5)$ and if $d_{1} \geq 7$ and $d_{2} \geq 6$ then $\left(m_{1}, m_{2}, m_{3}\right)=(8,84,84)$ and $(\Sigma, \tilde{c})=(176,244) ;$ or if $d_{1}=5$ and $d_{2} \geq 6$ then $\left(m_{1}, m_{2}, m_{3}\right)=(84,84,77 / 2)$ and $(\Sigma, \tilde{c})=$ $(413 / 2,244)$; or if $d_{2}=4$ and $\left(d_{1}, d_{3}, d_{4}\right)=(5,7,7)$ or $(7,7,5)$ or $(7,5,7)$ then $\left(m_{1}, m_{2}, m_{3}\right)=$ $(39,84,84)$ and $(\Sigma, \tilde{c})=(207,222)$; or if $d_{2}=4$ and $d_{3}=d_{4}=5$ then $\left(m_{1}, m_{2}, m_{3}\right)=(39,49,84)$ and $(\Sigma, \tilde{c})=(172,174)$; or if $d_{2}=4$ and $d_{1}=d_{3}=5$ then $\left(m_{1}, m_{2}, m_{3}\right)=(63 / 2,49,84)$ and $(\Sigma, \tilde{c})=(329 / 2,174)$. For $(1,4,9)$ labelling prevents $d_{3}=d_{4}=5$ so $\left(d_{1}, d_{2}, d_{3}, d_{4}\right)=$ $(5,5,5 / 7,6 / 5)$ and if $d_{3} \geq 7$ then $\left(m_{1}, m_{2}, m_{4}\right)=(84,36,84)$ and $(\Sigma, \tilde{c})=(204,216)$; or if $d_{3}=5$ and $d_{2} \geq 7$ then $\left(m_{1}, m_{2}, m_{4}\right)=(39,84,84)$ and $(\Sigma, \tilde{c})=(207,244)$; or if $d_{2}=d_{3}=5$ then $\left(m_{1}, m_{2}, m_{4}\right)=(42,84,56)$ (see Figure 3.11(iii)) and $(\Sigma, \tilde{c})=(182,196)$. For $(2,3,8), l \neq 2 k$ prevents $d_{3}=d_{4}=5$ so $\left(d_{1}, d_{2}, d_{3}, d_{4}\right)=(5,4,5 / 7,6 / 5)$ and if $d_{2} \geq 6$ then $\left(m_{1}, m_{2}, m_{4}\right)=$ $(56,84,84)$ and $(\Sigma, \tilde{c})=(224,224)$; or if $d_{2}=4$ and $d_{1} \geq 7$ then $\left(m_{1}, m_{2}, m_{4}\right)=(39,84,46)$ and $(\Sigma, \tilde{c})=(169,202)$; or if $d_{1}=5, d_{2}=4$ and $d_{3} \geq 7$ then $\left(m_{1}, m_{2}, m_{4}\right)=(63 / 2,56,84)$ and $(\Sigma, \tilde{c})=(343 / 2,234)$; or if $d_{1}=d_{3}=5$ and $d_{2}=4$ then $\left(m_{1}, m_{2}, m_{4}\right)=(63 / 2,49,56)$ and $(\Sigma, \tilde{c})=(273 / 2,154)$. For $(1,6,9)$ we have $\left(d_{1}, d_{2}, d_{3}, d_{4}\right)=(5,5,6,5)$ and if $d_{2} \geq 7$ then 


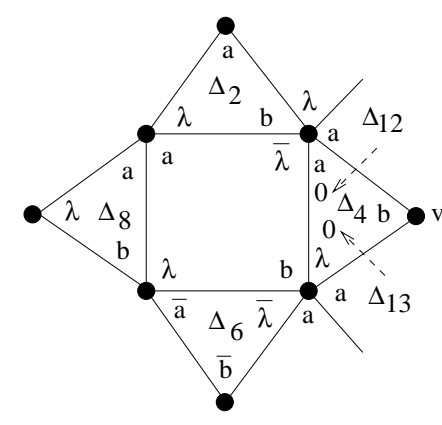

(i)

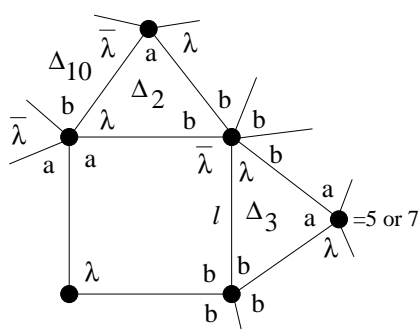

(iv)

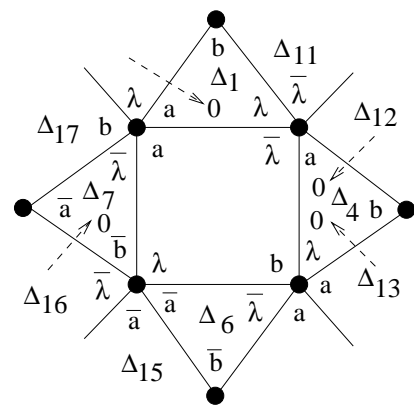

(ii)

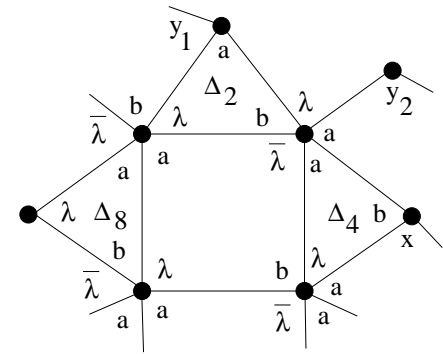

(v)

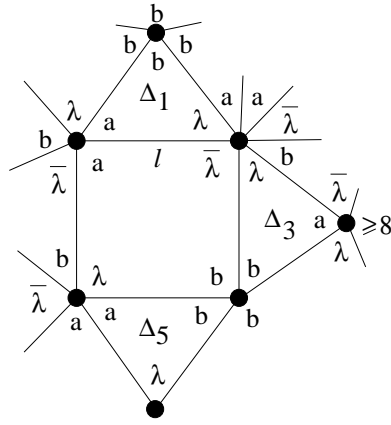

(iii)

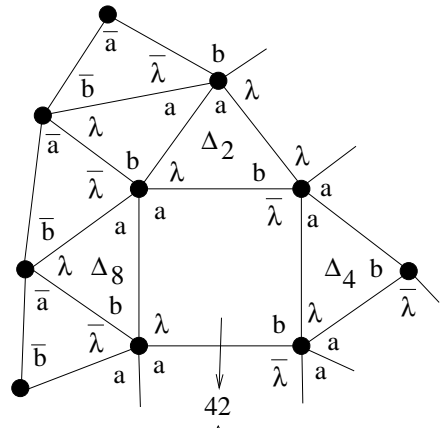

$\hat{\Delta}_{1}$

(vi)

Figure 3.14: degree 3 neighbours of a degree 4 region for $n=8$

$\left(m_{1}, m_{3}, m_{4}\right)=(39,84,84)$ and $(\Sigma, \tilde{c})=(207,244)$; or if $d_{2}=6$ then $\left(m_{1}, m_{3}, m_{4}\right)=(56,56,84)$ and $(\Sigma, \tilde{c})=(196,224)$; or if $d_{2}=5$ ( see Figure 3.11(iii)) then $\left(m_{1}, m_{3}, m_{4}\right)=(42,56,84)$ and $(\Sigma, \tilde{c})=(182,196)$. For $(2,7,8)$ we have $\left(d_{1}, d_{2}, d_{3}, d_{4}\right)=(5,4,5,6)$ and if $d_{2} \geq 6$ then $\left(m_{1}, m_{3}, m_{4}\right)=(84,56,56)$ and $(\Sigma, \tilde{c})=(196,244)$; or if $d_{2}=4$ then $\left(m_{1}, m_{3}, m_{4}\right)=(39,56,56)$ and $(\Sigma, \tilde{c})=(151,154)$. For $(3,5,8)$ we obtain $l=2 k$ a contradiction. Finally for $(3,6,9)$ we have $\left(d_{1}, d_{2}, d_{3}, d_{4}\right)=(5,4,7,5)$ and if $d_{2} \geq 6$ then $\left(m_{2}, m_{3}, m_{4}\right)=(84,36,84)$ and $(\Sigma, \tilde{c})=(204,244)$; or if $d_{2}=4$ and $d_{3}=7$ then $\left(m_{2}, m_{3}, m_{4}\right)=(25,36,84)$ and $(\Sigma, \tilde{c})=(145,174)$; or if $d_{2}=4$ and $d_{3} \geq 8$ then $\left(m_{2}, m_{3}, m_{4}\right)=(56,21,84)$ and $(\Sigma, \tilde{c})=(161,189)$.

Now suppose that $\hat{\Delta}$ is given by Figure 2.1(vi). Suppose that the four neighbouring regions of $\hat{\Delta}$ are 3 -gons. We see from Figure 3.13 that there are 16 possibilities. For 9 cases the values of $(\Sigma, \tilde{c})$ are straightforward and are as follows: $(1,3,6,7),(5,7,6,5),(78,8,56,84),(226,244) ;(1,3,6,8)$, $(8,7,6,7),(84,84,84,84),(336,355) ;(1,4,5,7),(5,7,6,7),(84,84,22,84),(274,292) ;(1,4,6,8)$, $(8,7,5,7),(84,84,84,0),(252,327) ;(2,3,5,7),(8,6,4,7),(0,84,84,0),(168,212) ;(2,3,6,7)$, $(8,6,6,5),(84,28,84,84),(280,287) ;(2,3,6,8),(5,6,6,7),(84,28,15,36),(163,272) ;(2,4,5,7)$, $(8,5,6,7),(84,84,84,0),(252,307) ;$ and $(2,4,6,7),(8,5,5,5),(21,84,84,21),(210,231)$. If $(1,3,5,8),(1,4,5,8),(2,3,5,8)$ or $(2,4,5,8)$ then $l=k$, a contradiction. For $(1,3,5,7)$ we have $\left(d_{1}, d_{2}, d_{3}, d_{4}\right)=(5,7,4,7)$ and if $d_{3} \geq 6$ then $\left(m_{1}, m_{2}, m_{3}, m_{4}\right)=(78,8,84,84)$ and $(\Sigma, \tilde{c})=(254,292) ;$ or if $d_{3}=4$ and $d_{4}=7$ then $\left(m_{1}, m_{2}, m_{3}, m_{4}\right)=(78,63,25,36)$ and $(\Sigma, \tilde{c})=(202,222) ;$ or if $d_{3}=4$ and $d_{4} \geq 8$ then $\left(m_{1}, m_{2}, m_{3}, m_{4}\right)=(78,63,56,21)$ and $(\Sigma, \tilde{c})=(218,237)$. For $(2,4,6,8)$ we have $\left(d_{1}, d_{2}, d_{3}, d_{4}\right)=(5,5,5,7)$ and if either $d_{1} \geq 7$ or $d_{2} \geq 7$ or $d_{3} \geq 7$ then $\left(m_{1}, m_{2}, m_{3}, m_{4}\right)=(84,84,36,36)$ and $(\Sigma, \tilde{c})=(240,264)$; or if $d_{1}=d_{2}=d_{3}=5$ and if either $d\left(\Delta_{12}\right)>3$ or $d\left(\Delta_{13}\right)>3$ in Figure 3.14(i) then since $\Delta_{4}$ does not receive any curvature from $\Delta_{12}$ or $\Delta_{13}$ we have $\left(m_{1}, m_{2}, m_{3}, m_{4}\right)=(84,42,36,36)$ 
and $(\Sigma, \tilde{c})=(198,216)$, otherwise $d\left(\Delta_{12}\right)=d\left(\Delta_{13}\right)=3$ forces the third vertex of $\Delta_{4}$ to have degree $\geq 6$ so $\left(m_{1}, m_{2}, m_{3}, m_{4}\right)=(84,56,36,36)$ and $(\Sigma, \tilde{c})=(216,216)$. Finally, for $(1,4,6,7)$ we have $\left(d_{1}, d_{2}, d_{3}, d_{4}\right)=(5,7,5,5)$ and if $d_{1} \geq 7$ then $\left(m_{1}, m_{2}, m_{3}, m_{4}\right)=(30,84,84,36)$ and $(\Sigma, \tilde{c})=(234,264) ;$ or if $d_{3} \geq 7$ then $\left(m_{1}, m_{2}, m_{3}, m_{4}\right)=(84,15,36,84)$ and $(\Sigma, \tilde{c})=(219,264)$; or if $d_{4} \geq 7$ then $\left(m_{1}, m_{2}, m_{3}, m_{4}\right)=(84,84,30,36)$ and $(\Sigma, \tilde{c})=(240,264)$; or if $d_{1}=d_{3}=$ $d_{4}=5$ then $\Delta$ is shown in Figure 3.14(ii) in which the corner label $\lambda^{-1}$ of $\Delta_{11}$ is forced by Lemma 2.1(ii) and so $d\left(\Delta_{11}\right)>3$. In particular, Figure 3.9 (viii) does not apply to $\Delta_{1}$ and so $m_{1}=78 / 2=39$. (This observation for $\Delta_{1}$ and $\Delta_{4}$ will be used throughout what follows often without further explanation.) If $k>l$ then $d\left(\Delta_{15}\right)>3$ and $d\left(\Delta_{17}\right)>3$ so $m_{3}=60$ and this is the 60 sent to $\hat{\Delta}_{9}$ in Figure $3.7(\mathrm{iv})$, and $m_{4}=42$ since the only way this can be exceeded is if $\hat{\Delta}=\hat{\Delta}_{6}$ of Figure $3.8(\mathrm{v})$, but this contradicts $d\left(\Delta_{1}\right)=3$ in Figure 3.14(ii). Therefore if $d_{2}=7$ then $\left(m_{1}, m_{2}, m_{3}, m_{4}\right)=(39,36,60,42)$ and $(\Sigma, \tilde{c})=(177,216)$; or if $d_{2} \geq 8$ then $\left(m_{1}, m_{2}, m_{3}, m_{4}\right)=(39,84,60,42)$ and $(\Sigma, \tilde{c})=(225,231)$. If $k<l$ then $x=\lambda^{-1}$ in Figure 3.14(ii) so $d\left(\Delta_{13}\right)>3$ and, moreover, either $d\left(\Delta_{15}\right)>3$ or $d\left(\Delta_{16}\right)>3$ so $\left(m_{1}, m_{2}, m_{3}, m_{4}\right)=(39,18,84 / 42,42 / 84)$ and $(\Sigma, \tilde{c})=(183,216)$.

Now suppose that exactly three of the neighbouring regions of $\hat{\Delta}$ are 3 -gons. There (see Figure $3.13)$ are 32 possibilities. For 20 cases the values $(\Sigma, \tilde{c})$ are straightforward and are as follows: $(1,3,6),(5,7,6,5),(84,8,84,-),(176,244) ;(1,4,5),(5,7,6,5),(84,35 / 2,84,-),(371 / 2,244)$; $(1,4,6),(5,7,5,5),(39,84,84,-),(207,216)$ (see Figure 3.14 (ii)); $(2,3,6),(5,6,6,5),(84,28,84,-)$, $(196,244) ;(2,4,5),(5 / 7,5,6,7 / 5)$ (since $l \neq k$ prevents $\left.d_{1}=d_{4}=5\right),(84,56,84,-),(224,224)$; $(1,3,8),(8,7,4,5),(15,84,-, 84),(183,237) ;(1,4,7),(5,7,5,5),(39,84,-, 84),(207,216)$ (see Figure 3.14(ii)); $(1,4,8),(8,7,5,5),(84,84,-, 84),(252,279) ;(2,3,7),(8,6,4,5),(0,84,-, 84)$, $(168,237) ;(2,4,7),(8,5,5,5),(21,84,-, 84),(189,231) ;(1,5,7),(5,6,4,7),(78,-, 84,36),(198,202)$; $(1,6,8),(8,6,5,7),(84,-, 84,84),(252,307) ;(2,5,7),(8,5,4,7),(84,-, 84,0),(168,237) ;(2,6,7)$, $(8,5,5,5),(21,-, 84,84),(189,231) ;(2,8,6),(5,5,5,7),(84,-, 84,36),(204,216) ;(3,5,7),(5,6,4,7)$, $(-, 84,56,36),(176,202) ;(3,6,7),(5,6,6,5),(-, 28,84,84),(196,224) ;(3,6,8),(5,6,6,7),(-, 84,84,84)$, $(252,272) ;(4,5,7),(5,5,6,7),(-, 84,22,84),(190,244) ;$ and $(4,6,8),(5,5,5,7),(-, 84,84,36)$, $(204,216)$.

If $(1,5,8),(2,5,8),(3,5,8)$ or $(4,5,8)$ occurs then $l=k$, a contradiction. The remaining eight cases are dealt with in turn. For $(1,3,5)$ we have $\left(d_{1}, d_{2}, d_{3}, d_{4}\right)=(5,7,4,5)$ and if $d_{3} \geq 6$ then $\left(m_{1}, m_{2}, m_{3}\right)=(84,8,84)$ and $(\Sigma, \tilde{c})=(176,244)$; if $d_{3}=4$ and $d_{4} \geq 7$ then $\left(m_{1}, m_{2}, m_{3}\right)=$ $(78,63,56)$ and $(\Sigma, \tilde{c})=(197,222)$; if $d_{3}=4$ and $d_{1} \geq 7$ then $\left(m_{1}, m_{2}, m_{3}\right)=(30,63,49)$ and $(\Sigma, \tilde{c})=(142,222)$; if $d_{3}=4$ and $d_{1}=d_{4}=5$ and $d_{2} \geq 8$ then $\left(m_{1}, m_{2}, m_{3}\right)=(154 / 3,63,49)$ and $(\Sigma, \tilde{c})=(490 / 3,189)$; or if $d_{3}=4$ and $d_{1}=d_{4}=5$ and $d_{2}=7$ and the third vertex, $\mathrm{v}$ say, of $\Delta_{1}$ does not have label $b^{4}$ then $\left(m_{1}, m_{2}, m_{3}\right)=(36,57,49)$ and $(\Sigma, \tilde{c})=(142,174)$; or if $d_{3}=4, d_{1}=d_{4}=5, d_{2}=7$ and $l(v)=b^{4}$ then this forces the third vertex of $\Delta_{3}$ to have degree $\geq 8$ (see Figure $3.14($ iii) $)$ and so $\left(m_{1}, m_{2}, m_{3}\right)=(78,15,49)$ and $(\Sigma, \tilde{c})=(142,174)$. For $(2,3,5)$ we cannot have $d_{1}=d_{4}=5$ so $\left(d_{1}, d_{2}, d_{3}, d_{4}\right)=(5 / 7,6,4,7 / 5)$ and if $d_{3} \geq 6$ then $\left(m_{1}, m_{2}, m_{3}\right)=(84,28,84)$ and $(\Sigma, \tilde{c})=(196,272)$; if $d_{3}=4$ and $d_{1} \geq 7$ then $\left(m_{1}, m_{2}, m_{3}\right)=$ $(8,84,84)$ and $(\Sigma, \tilde{c})=(176,202)$; if $d_{3}=4, d_{1}=5$ and $d_{2} \geq 7$ then $\left(m_{1}, m_{2}, m_{3}\right)=(64,84,56)$ and $(\Sigma, \tilde{c})=(204,222)$; or if $d_{4}=4, d_{1}=5$ and $d_{2}=6$ and the third vertex of $\Delta_{3}$ has degree $\geq 8$ then $\left(m_{1}, m_{2}, m_{3}\right)=(84,35,56)$ and $(\Sigma, \tilde{c})=(175,202)$, while if the third vertex of $\Delta_{3}$ has degree 5 or 7 then $k \neq 2 l$ forces $\Delta_{1}$ to be given by Figure 3.14 (iv) in which the corner label $\lambda^{-1}$ of $\Delta_{10}$ is forced so $\left(m_{1}, m_{2}, m_{3}\right)=(0,84,56)$ and $(\Sigma, \tilde{c})=(140,202)$. For $(2,4,6)$ we cannot have $d_{1}=d_{4}=5$ so $\left(d_{1}, d_{2}, d_{3}, d_{4}\right)=(5 / 7,5,5,7 / 5)$ and if $d_{1} \geq 7$ then $\left(m_{1}, m_{2}, m_{3}\right)=(36,84,84)$ and $(\Sigma, \tilde{c})=(204,216)$; or if $d_{1}=5$ then $\left(m_{1}, m_{2}, m_{3}\right)=(84,84,36)$ and $(\Sigma, \tilde{c})=(204,216)$. For $(1,3,7)$ we have $\left(d_{1}, d_{2}, d_{3}, d_{4}\right)=(5,7,4,5)$ and if $d_{3} \geq 6$ then $\left(m_{1}, m_{2}, m_{4}\right)=(84,8,84)$ and $(\Sigma, \tilde{c})=(176,244) ;$ or if $d_{3}=4$ then this forces $d_{4} \geq 6$ so $\left(m_{1}, m_{2}, m_{4}\right)=(78,63,56)$ and $(\Sigma, \tilde{c})=(197,202)$. For $(2,3,8)$ we have $\left(d_{1}, d_{2}, d_{3}, d_{4}\right)=(5,6,4,5)$ and if $d_{3} \geq 6$ then $\left(m_{1}, m_{2}, m_{4}\right)=(84,28,84)$ and $(\Sigma, \tilde{c})=(196,224)$; or if $d_{3}=4$ then this forces $d_{4} \geq 6$ and if also $d_{1} \geq 7$ then $\left(m_{1}, m_{2}, m_{4}\right)=(8,84,84)$ and $(\Sigma, \tilde{c})=(176,230) ;$ if $d_{3}=4, d_{1}=5$ and $d_{4} \geq 7$ 
then $\left(m_{1}, m_{2}, m_{4}\right)=(84,161 / 2,30)$ and $(\Sigma, \tilde{c})=(401 / 2,202)$; if $d_{3}=4, d_{1}=5$ and $d_{2} \geq 7$ then $\left(m_{1}, m_{2}, m_{4}\right)=(64,63,56)$ and $(\Sigma, \tilde{c})=(183,202)$; if $d_{3}=4, d_{1}=5$ and $d_{2}=6$ and the third vertex, $v$ say, of $\Delta_{3}$ has degree $\geq 8$ then $\left(m_{1}, m_{2}, m_{4}\right)=(84,35,56)$ and $(\Sigma, \tilde{c})=(175,182)$, or if $d_{3}=4, d_{1}=5$ and $d_{2}=6$ and $d(v)=5$ or 7 then (see Figure $\left.3.14(\mathrm{iv})\right)\left(m_{1}, m_{2}, m_{4}\right)=(0,84,56)$ and $(\Sigma, \tilde{c})=(140,182)$. For $(1,6,7)$ we have $\left(d_{1}, d_{2}, d_{3}, d_{4}\right)=(5,6,5,5)$ and if $d_{1} \geq 7$ or $d_{4} \geq 7$ then $\left(m_{1}, m_{3}, m_{4}\right)=(84,84,36)$ and $(\Sigma, \tilde{c})=(204,244)$; if $d_{1}=d_{4}=5$ and $d_{3} \geq 6$ then $\left(m_{1}, m_{3}, m_{4}\right)=(84,56,84)$ and $(\Sigma, \tilde{c})=(224,224)$; if $d_{1}=d_{3}=d_{4}=5$ then as for $(1,4,6,7)$ we do not have $d\left(\Delta_{15}\right)=d\left(\Delta_{16}\right)=3$ in Figure 3.14(ii) so either $d\left(\Delta_{15}\right)>3$ and $m_{3}=42$ since $\hat{\Delta} \neq$ $\hat{\Delta}_{9}$ of Figure 3.7 (iv) due to $d\left(\hat{\Delta}_{4}\right)=3$ in Figure $3.7\left(\right.$ iv) , or $d\left(\Delta_{16}\right)>3$ and $m_{4}=42$ since $\hat{\Delta} \neq \hat{\Delta}_{6}$ of Figure 3.8(v) due to $d\left(\Delta_{1}\right)=3$; therefore if $d_{2}=6$ then $\left(m_{1}, m_{3}, m_{4}\right)=(56,84 / 42,42 / 84)$ and $(\Sigma, \tilde{c})=(182,196)$ or $d_{2} \geq 7,\left(m_{1}, m_{3}, m_{4}\right)=(78,84 / 42,42 / 84)$ and $(\Sigma, \tilde{c})=(204,216)$. For $(4,6,7)$ labellings prevents we $d_{1}=d_{2}=5$ so $\left(d_{1}, d_{2}, d_{3}, d_{4}\right)=(5 / 7,7 / 5,5,5)$ and if $d_{3} \geq 7$ or $d_{4} \geq 7$ then $\left(m_{2}, m_{3}, m_{4}\right)=(84,84,84)$ and $(\Sigma, \tilde{c})=(252,264) ;$ or if $d_{3}=d_{4}=5$ and $d_{1} \geq 7$ then $\left(m_{2}, m_{3}, m_{4}\right)=(84,84,36)$ and $(\Sigma, \tilde{c})=(204,216)$; so let $d_{1}=d_{3}=d_{4}=5$. As in Figure 3.14 (ii) for $(1,4,6,7)$ we cannot have $d\left(\Delta_{15}\right)=d\left(\Delta_{16}\right)=3$ and if $d\left(\Delta_{15}\right)>3$ then $m_{3}=60$ (Figure 3.7 (iv) $)$ so if $d_{2}=7$ then $\left(m_{2}, m_{3}, m_{4}\right)=(36,60,84)$ and $(\Sigma, \tilde{c})=(180,216)$; or if $d\left(\Delta_{15}\right)>3$ and $d_{2} \geq 8$ then $\left(m_{2}, m_{3}, m_{4}\right)=(84,60,84)$ and $(\Sigma, \tilde{c})=(228,231)$; or if $d\left(\Delta_{16}\right)>3$ then $m_{4}=56$ (Figure $\left.3.8(\mathrm{v})\right)$ so if $d_{2}=7$ then $\left(m_{2}, m_{3}, m_{4}\right)=(36,84,56)$ and $(\Sigma, \tilde{c})=(176,216)$; or if $d\left(\Delta_{16}\right)>3$ and $d_{2} \geq 8$ then $\left(m_{2}, m_{3}, m_{4}\right)=(84,84,56)$ and $(\Sigma, \tilde{c})=(224,231)$. Finally for $(2,4,8)$ we have $\left(d_{1}, d_{2}, d_{3}, d_{4}\right)=(5,5,5,5)$ and if $d_{2}=7$ then $\left(m_{1}, m_{2}, m_{4}\right)=(84,36,84)$ and $(\Sigma, \tilde{c})=(204,216)$; or if $d_{2} \geq 8$ then $\left(m_{1}, m_{2}, m_{4}\right)=(105 / 2,84,84)$ and $(\Sigma, \tilde{c})=(441 / 2,231)$; if $d_{2}=5$ and $d_{1} \geq 7$ then $\left(m_{1}, m_{2}, m_{4}\right)=(84,84,46)$ and $(\Sigma, \tilde{c})=(214,216)$; if $d_{1}=d_{2}=5$ and $d_{3} \geq 7$ then $\left(m_{1}, m_{2}, m_{4}\right)=(84,36,84)$ and $(\Sigma, \tilde{c})=(204,216) ;$ if $d_{1}=d_{2}=5$ and $d_{3}=6$ then $l \neq k$ forces $d_{4} \geq 6$ so $\left(m_{1}, m_{2}, m_{4}\right)=(84,56,84)$ and $(\Sigma, \tilde{c})=(224,224)$; if $d_{1}=d_{2}=d_{3}=5$ and $d_{4} \geq 7$ then $\left(m_{1}, m_{2}, m_{4}\right)=(84,84,36)$ and $(\Sigma, \tilde{c})=(204,216)$. This leaves $d_{i}=5(1 \leq i \leq 3)$, $d_{4}=5$ or 6 and $\Delta$ is given by Figure $3.14(\mathrm{v})$ where if $x=b$ then $l<k$ and this together with $k \neq 2 l$ forces $y_{1}=y_{2}=\lambda^{-1}$ so $\left(m_{1}, m_{2}, m_{4}\right)=(42,42,84)$ and $(\Sigma, \tilde{c})=(168,168)$, so assume that $x=\lambda^{-1}$. Then $m_{2}=42$ and so if $d_{4}=6$ then $\left(m_{1}, m_{2}, m_{4}\right)=(84,42,56)$ and $(\Sigma, \tilde{c})=(182,198)$, therefore let $d_{4}=5$. If either $m_{1}=42$ or $m_{4}=42$ then $\left(m_{1}, m_{2}, m_{4}\right)=(42,42,84)$ or $(84,42,42)$ and $(\Sigma, \tilde{c})=(168,168)$. The only way $m_{1}>42$ and $m_{4}>42$ can be achieved is shown in Figure 3.14 (vi) for which $\left(m_{1}, m_{2}, m_{4}\right)=(84,42,84)$ and $(\Sigma, \tilde{c})=(210,168)$. In this case we introduce a new distribution rule, namely add $c^{*}(\hat{\Delta}) \leq 42$ to $c\left(\hat{\Delta}_{1}\right)$ as shown. We will consider the region $\hat{\Delta}_{1}$ later in this subsection.

Now let $\hat{\Delta}=\hat{\Delta}_{A}$ of Figure 3.8(v) or (vi) and so $\hat{\Delta}$ is given by Figure 3.15(i) in which $d\left(\Delta_{18}\right)>3$ and as already noted, $m_{1}=56$. Therefore if exactly two of the neighbouring regions of $\hat{\Delta}$ have degree 3 and if $d_{3} \geq 5$ then $(\Sigma, \tilde{c})=(168,168)$; or if $d_{3}=4$ then $x=\lambda^{-1}$ and $y=\lambda$ in Figure $3.15(\mathrm{i})$ and $(\Sigma, \tilde{c})=(120,182)$. Suppose that all four neighbouring regions have degree 3. For $(2,3,6,8)$ we have $\left(d_{1}, d_{2}, d_{3}, d_{4}\right)=(5,6,6,7),\left(m_{1}, m_{2}, m_{3}, m_{4}\right)=(56,28,15,64)$ and $(\Sigma, \tilde{c})=(163,272)$. For $(2,4,6,8)$ we have $\left(d_{1}, d_{2}, d_{3}, d_{4}\right)=(5,5,5,7)$ and if $d_{3} \geq 7$ then $\left(m_{1}, m_{2}, m_{3}, m_{4}\right)=(56,84,15,64)$ and $(\Sigma, \tilde{c})=(219,264)$; or if $d_{3}=5$ then the corner label $\lambda^{-1}$ of $\Delta_{13}$ in Figure 3.15(ii) is forced, otherwise $k=2 l$, therefore $\left(m_{1}, m_{2}, m_{3}, m_{4}\right)=$ $(56,42,36,64)$ and $(\Sigma, \tilde{c})=(198,216)$. If $(2,3,5,8)$ or $(2,4,5,8)$ then $k=l$. Now suppose that exactly three neighbours have degree 3 . If $(2,5,8)$ then $k=l$. For $(2,6,8)$ we have $\left(d_{1}, d_{2}, d_{3}, d_{4}\right)=(5,5,5,7),\left(m_{1}, m_{3}, m_{4}\right)=(56,84,64)$ and $(\Sigma, \tilde{c})=(204,216)$. For $(2,3,8)$ if we have $\left(d_{1}, d_{2}, d_{3}, d_{4}\right)=(5,6,6,5)$ then $\left(m_{1}, m_{3}, m_{4}\right)=(56,28,112)$ and $(\Sigma, \tilde{c})=(196,224)$; or if $\left(d_{1}, d_{2}, d_{3}, d_{4}\right)=(5,6,4,6)$ then $\left(m_{1}, m_{3}, m_{4}\right)=(56,161 / 2,44)$ (see Figure 3.7(vi)) and $(\Sigma, \tilde{c})=(361 / 2,182) ;$ or if $\left(d_{1}, d_{2}, d_{3}, d_{4}\right)=(5,6,4,7)$ then $\left(m_{1}, m_{3}, m_{4}\right)=(56,161 / 2,64)$ (see Figure $3.8(\mathrm{vi}))$ and $(\Sigma, \tilde{c})=(401 / 2,202)$; For $(2,4,8)$ we have $\left(d_{1}, d_{2}, d_{3}, d_{4}\right)=(5,5,5,5)$ and if $d_{4}=6$ then $\left(m_{1}, m_{2}, m_{4}\right)=(56,84,44)$ (see Figure $3.7($ vi) ) and $(\Sigma, \tilde{c})=(184,196)$; if $d_{4} \geq 7$ then $\left(m_{1}, m_{2}, m_{4}\right)=(56,84,64)$ and $(\Sigma, \tilde{c})=(204,216)$; if $d_{4}=5, d_{2} \geq 7$ and $d_{3} \geq 7$ then $\left(m_{1}, m_{2}, m_{4}\right)=(56,84,112)$ and $(\Sigma, \tilde{c})=(252,266) ;$ if $d_{4}=d_{2}=5$ and $d_{3} \geq 7$ then $\left(m_{1}, m_{2}, m_{4}\right)=(56,36,112)$ and $(\Sigma, \tilde{c})=(204,216)$; or if $d_{4}=d_{3}=5$ and $d_{2} \geq 7$ then 


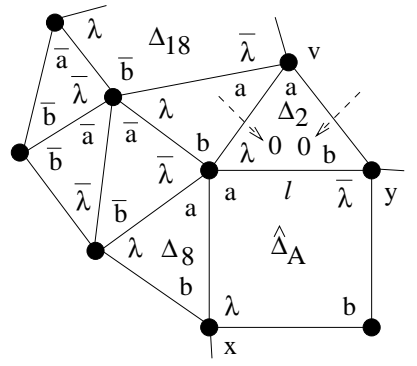

(i)

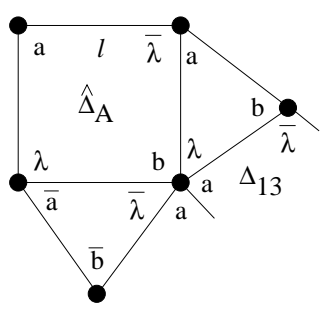

(ii)

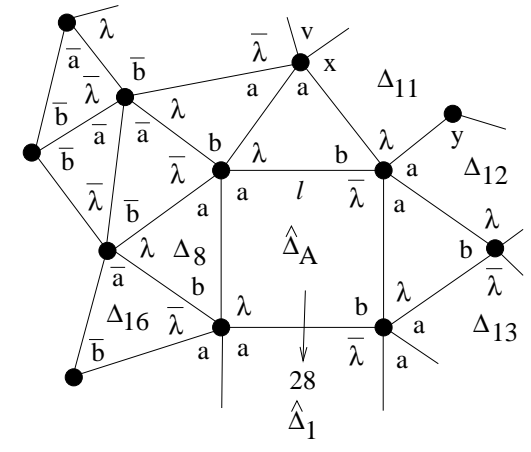

(iii)
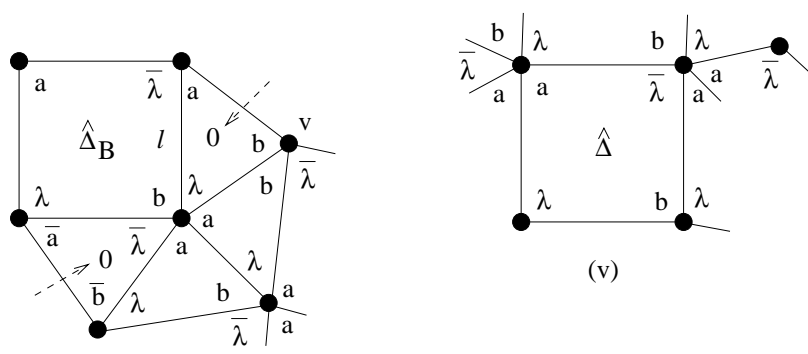

(v)

(iv)

Figure 3.15: curvature at regions $d\left(\hat{\Delta}_{A}\right)$ and $d\left(\hat{\Delta}_{B}\right)$

$d\left(\Delta_{13}\right)>3$ as in Figure 3.15(ii) so $\left(m_{1}, m_{2}, m_{4}\right)=(56,18,112)$ and $(\Sigma, \tilde{c})=(186,216)$. Since $d_{4}=5$ and $l \neq k$ prevents $d_{3}=6$ this leaves the case $d_{i}=5(1 \leq i \leq 4)$ and $\hat{\Delta}_{A}$ is shown in Figure 3.15(iii) in which if $x=a$ then $d(v) \geq 8$ and, moreover, the fact that $l<k$ then forces $y=\lambda^{-1}$ so $\left(m_{1}, m_{2}, m_{4}\right)=(21,28,112)$ and $(\Sigma, \tilde{c})=(161,168)$; if $x=\lambda$ and $d\left(\Delta_{16}\right)>3$ then $\left(m_{1}, m_{2}, m_{4}\right)=(42,42,84)$ and $(\Sigma, \tilde{c})=(168,168)$; or if $x=\lambda$ and $d\left(\Delta_{16}\right)=3$ as shown then $\left(m_{1}, m_{2}, m_{4}\right)=(42,42,112)$ and $(\Sigma, \tilde{c})=(196,168)$. Therefore we define the new distribution rule: add $c^{*}(\Delta) \leq 28$ to $c\left(\hat{\Delta}_{1}\right)$ as shown in Figure 3.15(iii).

Let $\hat{\Delta}=\hat{\Delta}_{B}$ and so $\hat{\Delta}$ is given by Figure 3.15(iv) and $k>l$. Note that if $d\left(u_{2}\right) \geq 7$ then $m_{2}=8$ or if $d\left(u_{2}\right)=5$ then $d(v) \geq 8$ and $m_{2}=21$. Therefore if exactly two of the neighbouring regions of $\hat{\Delta}$ have degree 3 then $(\Sigma, \tilde{c})=(133,168)$. Suppose that exactly three neighbouring of $\hat{\Delta}_{B}$ have degree 3 and the third is $\Delta$ say. If $\Delta=\Delta_{1}$ (of Figure 3.13) then $d_{2} \geq 7,\left(m_{1}, m_{2}, m_{3}\right)=$ $(84,8,112)$ and $(\Sigma, \tilde{c})=(204,216)$; if $\Delta=\Delta_{2}$ and $d_{4}=5$ then $d_{1} \geq 8,\left(m_{1}, m_{2}, m_{3}\right)=$ $(21,21,112)$ and $(\Sigma, \tilde{c})=(154,231)$, or if $d_{4} \geq 7,\left(m_{1}, m_{2}, m_{3}\right)=(84,21,64)$ and $(\Sigma, \tilde{c})=$ $(169,216)$; if $\Delta=\Delta_{7}$ and $d_{2}=5$ then $d_{1} \geq 8,\left(m_{2}, m_{3}, m_{4}\right)=(84,112,21)$ and $(\Sigma, \tilde{c})=$ $(217,231)$, or if $d_{2} \geq 7$ then $\left(m_{2}, m_{3}, m_{4}\right)=(8,112,84)$ and $(\Sigma, \tilde{c})=(204,216)$; or if $\Delta=$ $\Delta_{8}$ then $d_{4} \geq 7,\left(m_{2}, m_{3}, m_{4}\right)=(21,64,84)$ and $(\Sigma, \tilde{c})=(196,216)$. Now suppose that all four neighbouring regions have degree 3. For $(1,4,6,7)$ we have $d_{2} \geq 7$ so if $d_{1} \geq 7$ then $\left(m_{1}, m_{2}, m_{3}, m_{4}\right)=(30,8,112,36)$ and $(\Sigma, \tilde{c})=(186,264)$, or if $d_{1}=5$ then this forces $m_{1}=39$ and $m_{4}=42$ (see the previous analysis for $(1,4,6,7)$ and Figure $3.14($ ii) $)$ so $\left(m_{1}, m_{2}, m_{3}, m_{4}\right)=$ $(39,8,112,42)$ and $(\Sigma, \tilde{c})=(201,216)$. For $(1,4,6,8)$ we have $\left(d_{1}, d_{2}, d_{3}, d_{4}\right)=(8,7,5,7)$ so $\left(m_{1}, m_{2}, m_{3}, m_{4}\right)=(84,84,64,84)$ and $(\Sigma, \tilde{c})=(240,327)$. For $(2,4,6,7)$ we have $d_{1} \geq 8$, $\left(m_{1}, m_{2}, m_{3}, m_{4}\right)=(21,21,112,21)$ and $(\Sigma, \tilde{c})=(175,231)$. Finally for $(2,4,6,8)$ we have $d_{4} \geq 7,\left(m_{1}, m_{2}, m_{3}, m_{4}\right)=(84,21,64,36)$ and $(\Sigma, \tilde{c})=(205,216)$.

To complete the case $n=8$ observe that if $d(\hat{\Delta})=3$ then either $c^{*}(\hat{\Delta}) \leq 0$ or positive curvature is distributed from $\hat{\Delta}$ to a region of degree greater than 3 and so it remains to consider $\hat{\Delta}=\hat{\Delta}_{1}$ of 


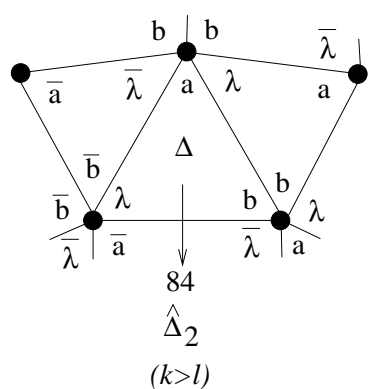

(i)

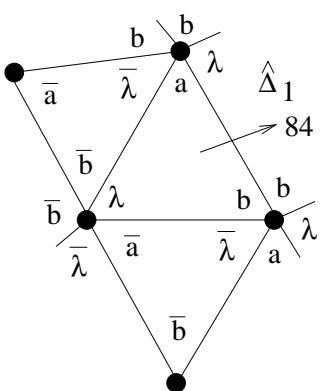

(ii)

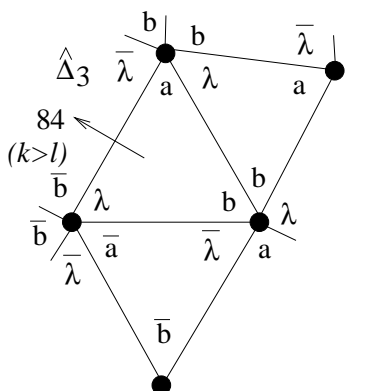

(iii)

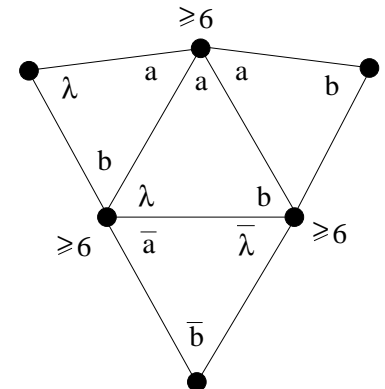

(iv)

Figure 3.16: curvature distribution for $n=7$

Figure 3.14(v) or 3.15(iii) in which case if $d(\hat{\Delta}) \geq 5$ then $c^{*}(\hat{\Delta}) \leq c(4,5,5,5,5)+4(84)+42=0$, so assume that $d(\hat{\Delta})=4$ as shown in Figure $3.15(\mathrm{v})$. If at most one of the neighbouring regions of $\hat{\Delta}$ has degree 3 then $c^{*}(\hat{\Delta}) \leq c(5,5,5,5)+42+84<0$. Suppose exactly two have degree 3. For $(4,5),(4,6)\left(\hat{\Delta} \neq \hat{\Delta}_{B}\right),(4,8)$, since $\hat{\Delta}$ receives at most 42 from $\Delta_{4}$ we have $c^{*}(\hat{\Delta}) \leq c(5,5,5,5)+2(42)+84=0$; for $(4,6)$ with $\hat{\Delta}=\hat{\Delta}_{B}, d_{4} \geq 7$ so we see from the above analysis for $\hat{\Delta}_{B}$ that $m_{2}=21$ and $m_{3}=64$ so $c^{*}(\hat{\Delta}) \leq c(5,5,5,7)+42+21+64=-216+127<0$; and for $(6,8), d_{4} \geq 7$ so $\hat{\Delta}$ receives at most 36 from $\Delta_{8}$ and $c^{*}(\hat{\Delta}) \leq c(5,5,5,7)+42+84+36<0$. If $(5,8)$ then $k=l$. Finally if there are exactly three 3 -gons then it must be $(4,6,8)$ so $d_{3} \geq 7$ and $c^{*}(\hat{\Delta}) \leq c(5,5,5,7)+42+42+84+36<0$ when $\hat{\Delta} \neq \hat{\Delta}_{B} ;$ and $c^{*}(\hat{\Delta}) \leq c(5,5,5,7)+42+$ $21+64+36<0$ when $\hat{\Delta}=\hat{\Delta}_{B}$.

\section{$3.4 n=7(l \neq k, l \neq 2 k, k \neq 2 l)$}

If $d(v) \leq 7$ then $l(v) \in\left\{a \lambda b^{2} \lambda^{-1}, a^{3} \lambda b^{-1} \lambda^{-1}, a \lambda b^{2} \lambda^{-1} \lambda \lambda^{-1}, a \lambda b \lambda^{-1} \lambda b \lambda^{-1}, a \lambda \lambda^{-1} \lambda b^{2} \lambda^{-1}, a^{2} \lambda b^{-3} \lambda^{-1}\right\}$ by Lemma 2.2. Again use will be made of the assumptions $l \neq k, l \neq 2 k$ and $k \neq 2 l$ often without explicit mention.

If $c(\Delta)>0$ then $\Delta$ is given by Figure 3.1(i). If $d\left(\hat{\Delta}_{i}\right)>3(1 \leq i \leq 3)$ then distribute $\frac{1}{3} c(\Delta)$ to each $c\left(\hat{\Delta}_{i}\right)$; if exactly one of the $\hat{\Delta}_{i}$ has degree 3 then distribute $\frac{1}{2} c(\Delta)$ to each of the other two neighbouring regions; or if exactly two of the $\hat{\Delta}_{i}$ have degree 3 then distribute $c(\Delta)$ to the third region. Given this, the maximum amount of curvature (in multiples of $\pi / 420$ ) crossing an edge according to the degrees of its endpoints is shown in Figure 3.5(i); and the three cases when 56 is exceeded are shown in Figure 3.16(i)-(iii).

Now let $d\left(\hat{\Delta}_{i}\right)=3(1 \leq i \leq 3)$ in Figure 3.1(i). Then $l \neq k$ implies that there are six possible configurations and these are shown in Figure 3.16(iv) and 3.17(i)-(v). If $\Delta$ is given by Figure 3.16(iv) then $c(\Delta) \leq 0$; or if by Figure 3.17(i) then $d\left(\hat{\Delta}_{4}\right)>3$, so if $d\left(\hat{\Delta}_{5}\right)>3$ then add $\frac{1}{2}\left(c(\Delta)+c\left(\hat{\Delta}_{1}\right)\right) \leq 22$ to each of $c\left(\hat{\Delta}_{4}\right)$ and $c\left(\hat{\Delta}_{5}\right)$ as shown in Figure 3.17(vi), or if $d\left(\hat{\Delta}_{5}\right)=3$ then $d\left(v_{4}\right) \geq 6$ and add $c(\Delta)+c\left(\hat{\Delta}_{1}\right) \leq 16$ to $c\left(\hat{\Delta}_{4}\right)$ as in Figure 3.17(vii).

Let $\Delta$ be given by Figure 3.17 (ii) in which case $d\left(v_{2}\right)=5$ otherwise $c(\Delta) \leq 0$. If $d\left(\hat{\Delta}_{8}\right)>3$ and $d\left(\hat{\Delta}_{9}\right)>3$ then add $\frac{1}{2}\left(c(\Delta)+c\left(\hat{\Delta}_{3}\right)\right) \leq 28$ to each of $c\left(\hat{\Delta}_{8}\right)$ and $c\left(\hat{\Delta}_{9}\right)$ as in Figure 3.18(i). Suppose otherwise and assume until otherwise stated that $d\left(v_{1}\right)=6$. If $d\left(\hat{\Delta}_{8}\right)>3, d\left(\hat{\Delta}_{9}\right)=3$ and $d\left(v_{6}\right) \geq 6$ then add $c(\Delta)+c\left(\hat{\Delta}_{3}\right) \leq 28$ to $c\left(\hat{\Delta}_{8}\right)$ as in Figure 3.18(ii); or if $d\left(\hat{\Delta}_{8}\right)>3$, $d\left(\hat{\Delta}_{9}\right)=3$ and $d\left(v_{6}\right)=5$ then add $c(\Delta)+c\left(\hat{\Delta}_{3}\right) \leq 56$ to $c\left(\hat{\Delta}_{8}\right)$ as in (iii). If $d\left(\hat{\Delta}_{8}\right)=3$, $d\left(\hat{\Delta}_{9}\right)>3$ and $\hat{\Delta}_{8}$ is given by Figure 3.18(iv) then $d\left(v_{3}\right)=7$ and $d\left(v_{6}\right) \geq 7$ so add $c(\Delta)=8$ to $c\left(\hat{\Delta}_{3}\right) \leq-40$ as shown; or if $\hat{\Delta}_{8}$ is given by $(\mathrm{v})$ then $d\left(v_{6}\right) \geq 6$ so add $c(\Delta)+c\left(\hat{\Delta}_{3}\right) \leq 28$ to 


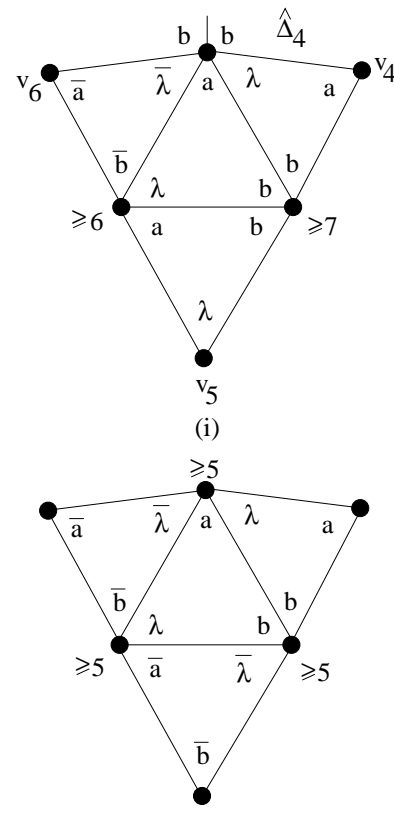

(v)

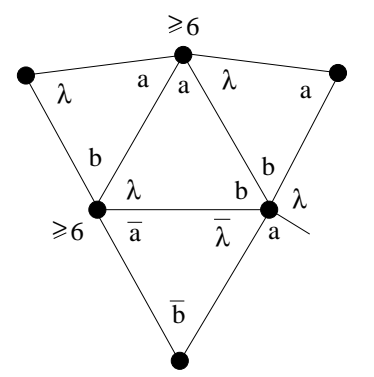

(ii)

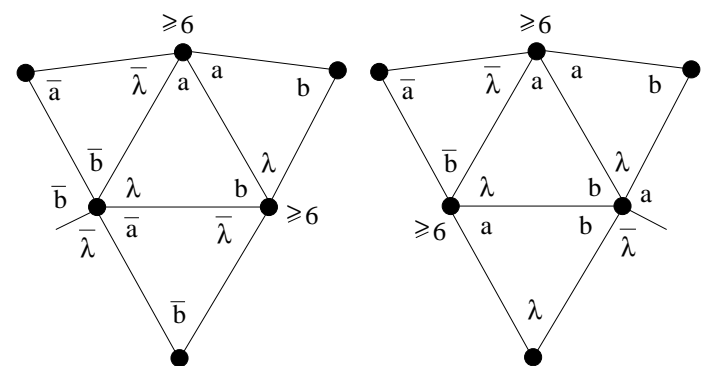

(iii)

(iv)

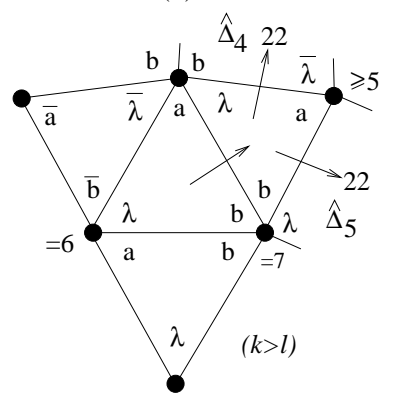

(vi)

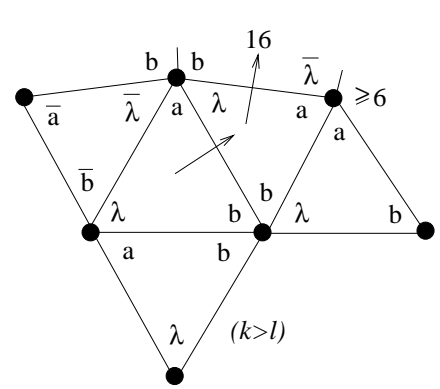

(vii)

Figure 3.17: three neighbouring regions of degree 3

$c\left(\hat{\Delta}_{9}\right)$ as shown. Let $d\left(\hat{\Delta}_{8}\right)=d\left(\hat{\Delta}_{9}\right)=3$, in which case $d\left(v_{3}\right) \neq 7$ so $c(\Delta)>0$ forces $d\left(v_{3}\right)=6$ and $d\left(v_{5}\right) \geq 6$. If $d\left(\hat{\Delta}_{6}\right)>3$ and $d\left(\hat{\Delta}_{7}\right)>3$ then add $\frac{1}{2}\left(c(\Delta)+c\left(\hat{\Delta}_{2}\right)\right) \leq 28$ to each of $c\left(\hat{\Delta}_{6}\right)$ and $c\left(\hat{\Delta}_{7}\right)$ as in Figure 3.18(vi); if $d\left(\hat{\Delta}_{6}\right)=3$ and $d\left(\hat{\Delta}_{7}\right)>3$ then add $c(\Delta)+c\left(\hat{\Delta}_{2}\right) \leq 56$ to $c\left(\hat{\Delta}_{7}\right)$ as in (vii); if $d\left(\hat{\Delta}_{6}\right)>3$ and $d\left(\hat{\Delta}_{7}\right)=3$ then add $c(\Delta)+c\left(\hat{\Delta}_{2}\right) \leq 56$ to $c\left(\hat{\Delta}_{6}\right)$ as in (viii). Let $d\left(\hat{\Delta}_{6}\right)=d\left(\hat{\Delta}_{7}\right)=3$, in which case $d\left(\hat{\Delta}_{5}\right)>3$. If $d\left(\hat{\Delta}_{4}\right)>3$ and $d\left(v_{4}\right)=5$ then add $\frac{1}{3}\left(c(\Delta)+c\left(\hat{\Delta}_{1}\right)\right)=28$ to $c\left(\hat{\Delta}_{4}\right)$ and $\frac{2}{3}\left(c(\Delta)+c\left(\hat{\Delta}_{1}\right)\right)=56$ to $c\left(\hat{\Delta}_{5}\right)$ as in Figure 3.18(ix); or if $d\left(v_{4}\right) \geq 6$ then add $\frac{1}{2}\left(c(\Delta)+c\left(\hat{\Delta}_{1}\right)\right) \leq 28$ to each of $c\left(\hat{\Delta}_{4}\right)$ and $c\left(\hat{\Delta}_{5}\right)$ as shown in (ix). If $d\left(\hat{\Delta}_{4}\right)=3$ and $d\left(v_{4}\right)=5, \geq 6$ then add $c(\Delta)+c\left(\hat{\Delta}_{1}\right)=84, \leq 56$ to $c\left(\hat{\Delta}_{5}\right)$ as in Figure 3.18(x). Now assume that $d\left(v_{1}\right)=7$ and $d\left(v_{3}\right)=6$. If $d\left(\hat{\Delta}_{8}\right)>3$ and $d\left(\hat{\Delta}_{9}\right)=3$ then add $c(\Delta)+c\left(\hat{\Delta}_{3}\right) \leq 16$ to $c\left(\hat{\Delta}_{8}\right)$ as in Figure $3.18(\mathrm{xi})$;

or if $d\left(\hat{\Delta}_{8}\right)=3$ and $d\left(\hat{\Delta}_{9}\right)>3$ then add $c(\Delta)+c\left(\hat{\Delta}_{3}\right) \leq 16$ to $c\left(\hat{\Delta}_{9}\right)$ as in (xii). Let $d\left(\hat{\Delta}_{8}\right)=$ $d\left(\hat{\Delta}_{9}\right)=3$. If $d\left(\hat{\Delta}_{6}\right)>3$ and $d\left(\hat{\Delta}_{7}\right)>3$ then add $\frac{1}{2}\left(c(\Delta)+c\left(\hat{\Delta}_{2}\right)\right) \leq 18$ to each of $c\left(\hat{\Delta}_{6}\right)$ and $c\left(\hat{\Delta}_{7}\right)$ as in Figure 3.19(i); if $d\left(\hat{\Delta}_{6}\right)>3, d\left(\hat{\Delta}_{7}\right)=3$ and $d\left(v_{5}\right)=6, \geq 7$ then add $c(\Delta)+c\left(\hat{\Delta}_{2}\right)=36$, $\leq 16$ (respectively) to $c\left(\hat{\Delta}_{6}\right)$ as in (ii); if $d\left(\hat{\Delta}_{6}\right)=3$ then $d\left(\hat{\Delta}_{5}\right)>3$ and if $d\left(\hat{\Delta}_{4}\right)>3$ and $d\left(v_{5}\right)=5$ then add $\frac{1}{3}\left(c(\Delta)+c\left(\hat{\Delta}_{1}\right)\right)=16$ to $c\left(\hat{\Delta}_{4}\right)$ and $\frac{2}{3}\left(c(\Delta)+c\left(\hat{\Delta}_{1}\right)\right)=28$ to $c\left(\hat{\Delta}_{5}\right)$ as in (iii); if $d\left(\hat{\Delta}_{6}\right), d\left(\hat{\Delta}_{4}\right)>3$ and $d\left(v_{5}\right) \geq 6$ then add $\frac{1}{2}\left(c(\Delta)+c\left(\hat{\Delta}_{1}\right)\right) \leq 8$ to each of $c\left(\hat{\Delta}_{4}\right)$ and $c\left(\hat{\Delta}_{5}\right)$ again as in (iii); or if $d\left(\hat{\Delta}_{6}\right)=d\left(\hat{\Delta}_{4}\right)=3$ and $d\left(v_{4}\right)=5, \geq 6$ then add $c(\Delta)+c\left(\hat{\Delta}_{1}\right)=44, \leq 16$ (respectively) to $c\left(\hat{\Delta}_{5}\right)$ as in (iv).

Let $\Delta$ be given by Figure 3.17(iii) in which case $c(\Delta)>0$ forces $d\left(v_{3}\right)=5$. If $d\left(\hat{\Delta}_{4}\right)>3$ and $d\left(\hat{\Delta}_{5}\right)>3$ then add $\frac{1}{2}\left(c(\Delta)+c\left(\hat{\Delta}_{1}\right)\right) \leq 28$ to each of $c\left(\hat{\Delta}_{4}\right)$ and $c\left(\hat{\Delta}_{5}\right)$ as shown in Figure $3.19(\mathrm{v})$; if $d\left(\hat{\Delta}_{5}\right)>3$ and $\hat{\Delta}_{4}$ is given by Figure 3.19(vi) then add $c(\Delta)+c\left(\hat{\Delta}_{1}\right) \leq 16$ to $c\left(\hat{\Delta}_{5}\right)$ as shown; if $d\left(\hat{\Delta}_{5}\right)>3, d\left(v_{2}\right)=7$ and $\hat{\Delta}_{4}$ is given by Figure 3.19 (vii) then add $c(\Delta)+c\left(\hat{\Delta}_{1}\right) \leq 16$ to $c\left(\hat{\Delta}_{5}\right)$ as shown; or if $d\left(\hat{\Delta}_{5}\right)>3, d\left(v_{2}\right)=6$ and $\hat{\Delta}_{4}$ is given by Figure 3.19 (viii) then $d\left(v_{4}\right) \geq 6$, and add $c(\Delta)+c\left(\hat{\Delta}_{1}\right) \leq 28$ to $c\left(\hat{\Delta}_{5}\right)$ as shown. Now assume that $d\left(\hat{\Delta}_{5}\right)=3$ in which case $\hat{\Delta}_{5}$ is given by Figure 3.19(ix) and this forces $d\left(v_{2}\right)=6$. If $d\left(\hat{\Delta}_{8}\right)>3$ and $d\left(\hat{\Delta}_{9}\right)>3$ and $d\left(v_{1}\right)=7, d\left(v_{6}\right) \geq 5$ 

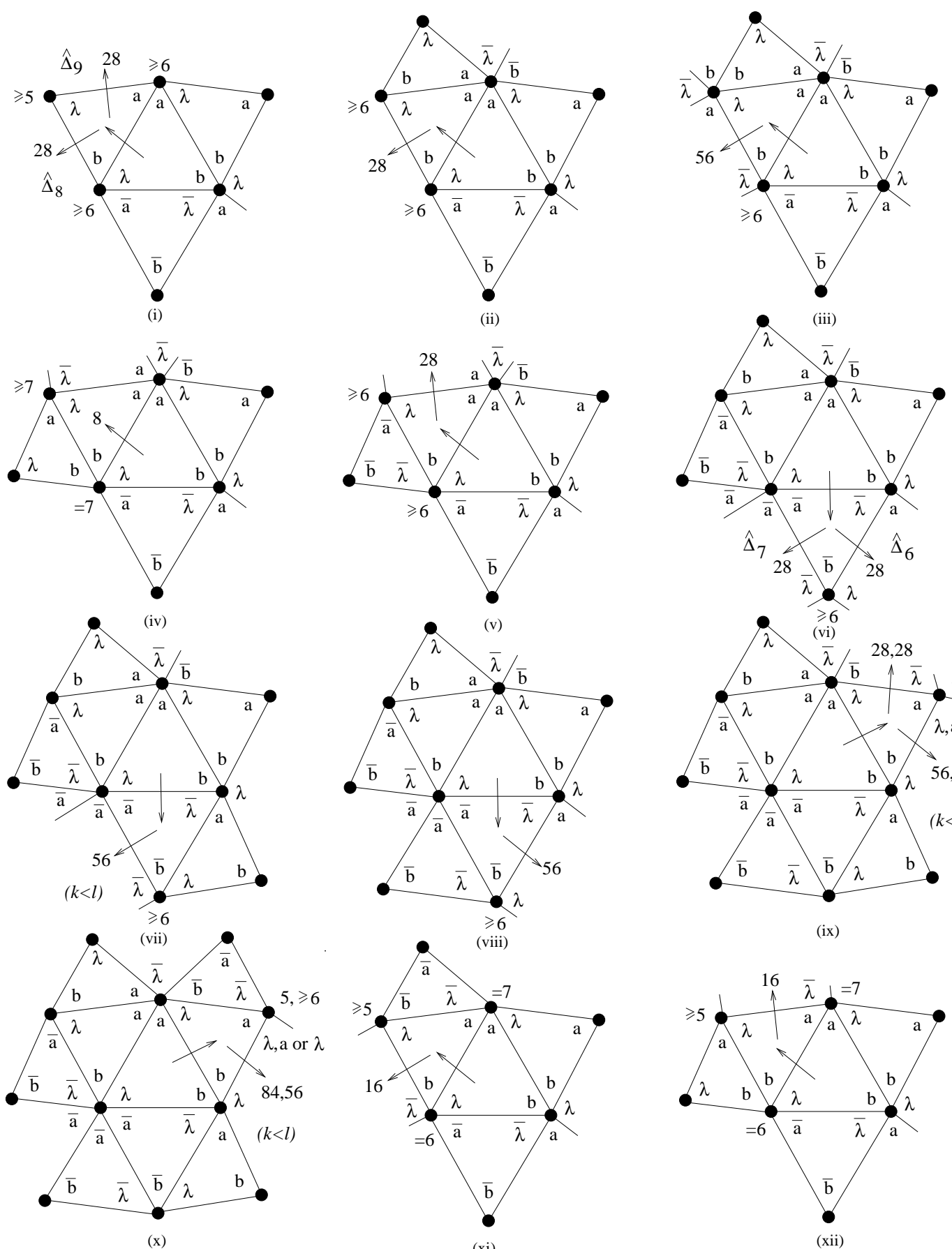

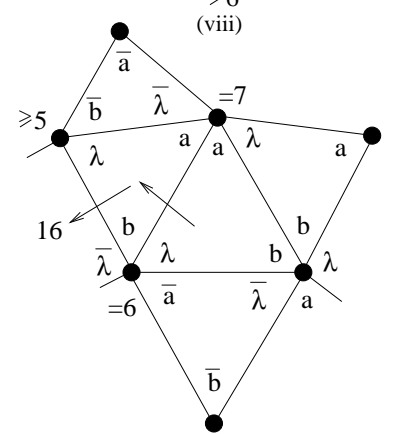

(xi)

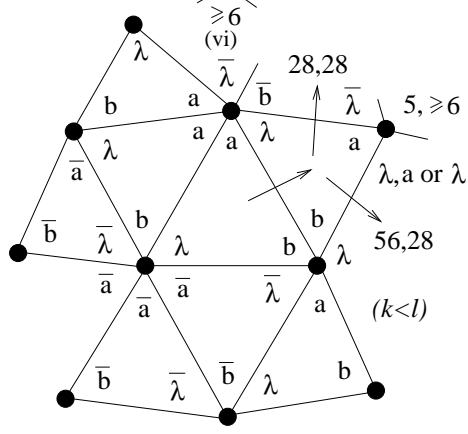

(ix)

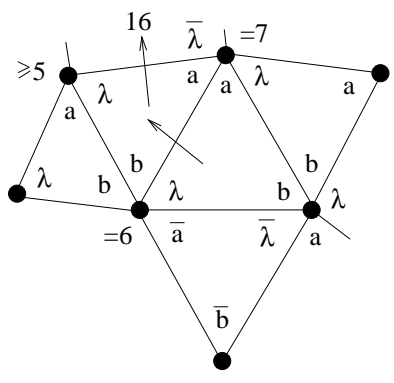

(xii)

Figure 3.18: curvature distribution for $n=7$ 

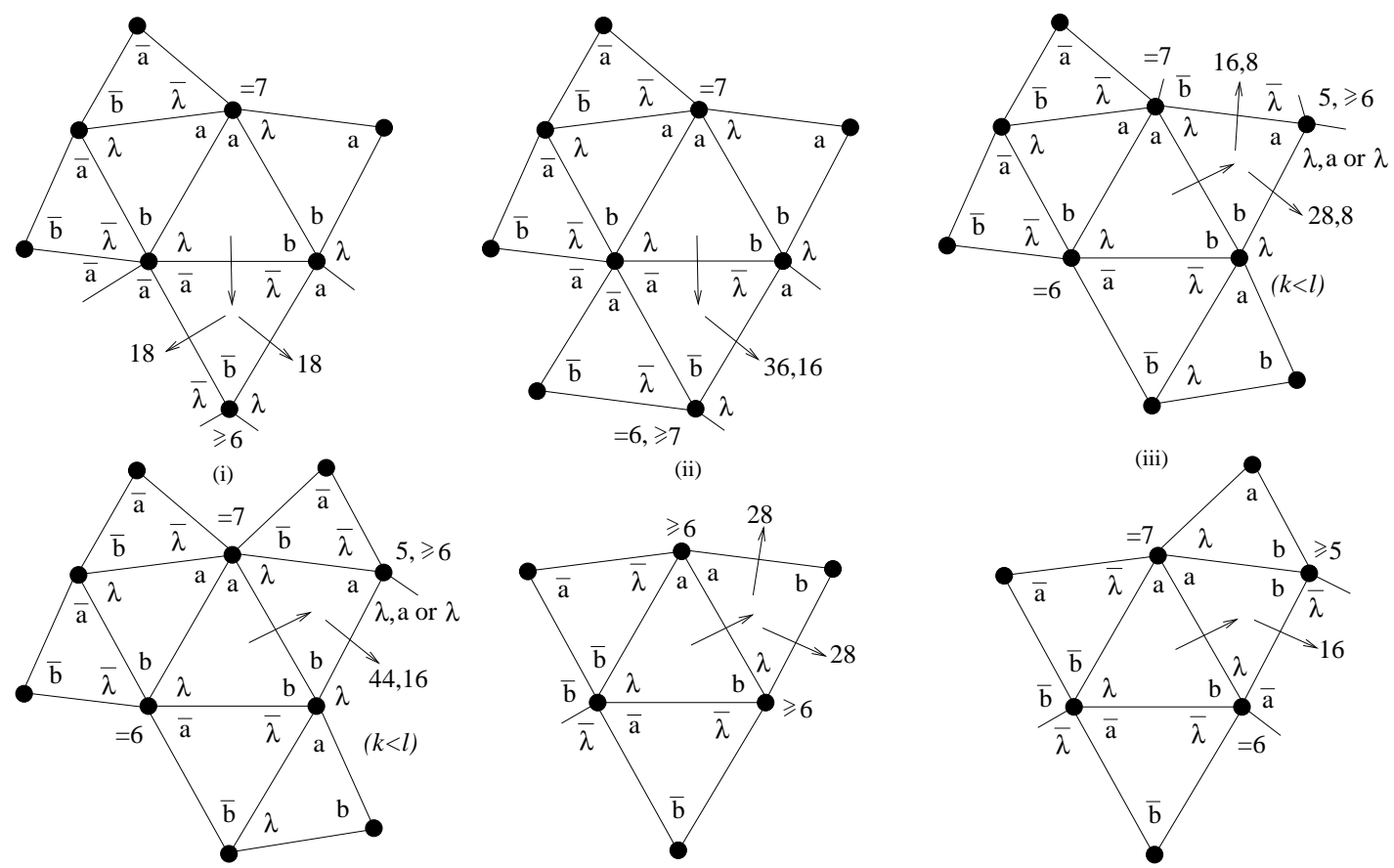

(ii)
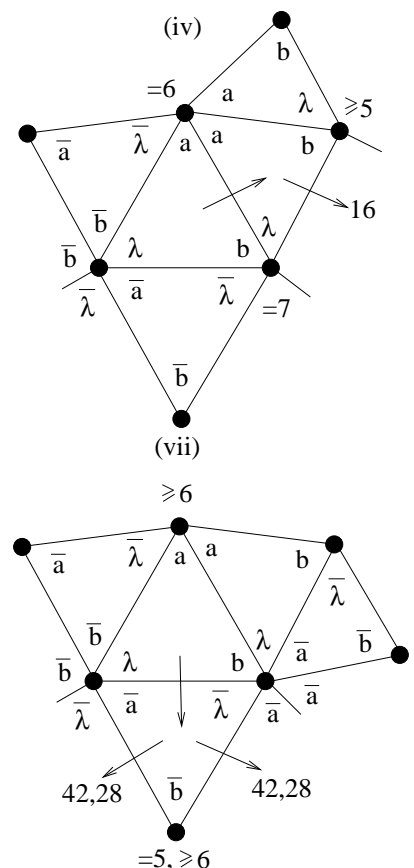

(x)

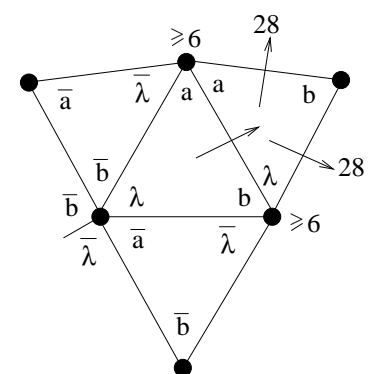

(v)

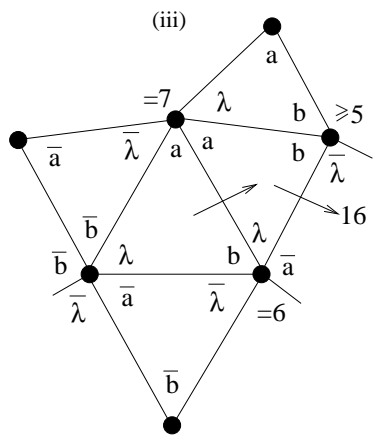

(vi)
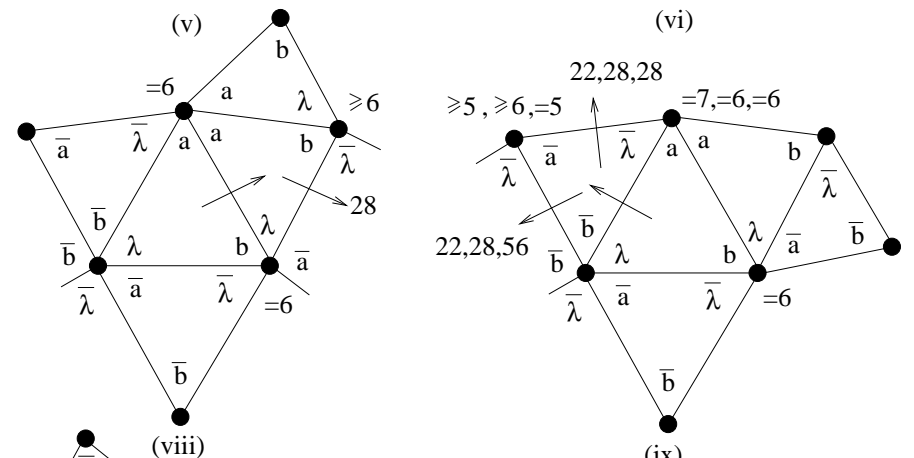

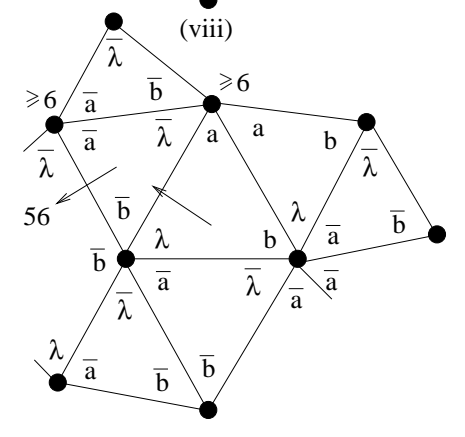

(xi)

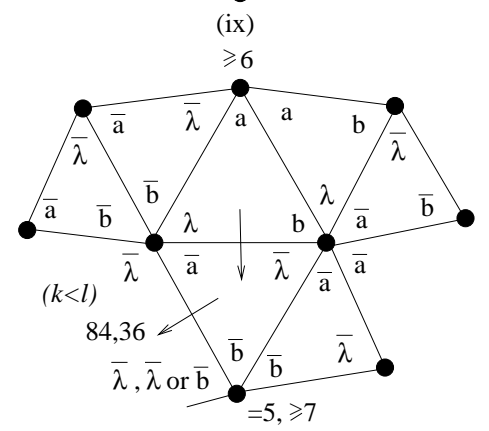

(xii)

Figure 3.19: curvature distribution for $n=7$ 
or $d\left(v_{1}\right)=6, d\left(v_{6}\right) \geq 6$ then add $\frac{1}{2}\left(c(\Delta)+c\left(\hat{\Delta}_{3}\right)\right) \leq 22$ or $\leq 28$ to each of $c\left(\hat{\Delta}_{8}\right)$ and $c\left(\hat{\Delta}_{9}\right)$, whereas if $d\left(v_{1}\right)=6, d\left(v_{6}\right)=5$ then add $\frac{1}{3}\left(c(\Delta)+c\left(\hat{\Delta}_{3}\right)\right)=28$ to $c\left(\hat{\Delta}_{9}\right)$ and $\frac{2}{3}\left(c(\Delta)+c\left(\hat{\Delta}_{3}\right)\right)=56$ to $c\left(\hat{\Delta}_{8}\right)$ as shown in Figure 3.19(ix). Suppose that at least one of $\hat{\Delta}_{8}$ or $\hat{\Delta}_{9}$ has degree 3. If $d\left(\hat{\Delta}_{6}\right)>3$ and $d\left(\hat{\Delta}_{7}\right)>3$ and $d\left(v_{5}\right)=5, \geq 6$ then add $\frac{1}{2}\left(c(\Delta)+c\left(\hat{\Delta}_{2}\right)\right) \leq 42, \leq 28$ (respectively) to each of $c\left(\hat{\Delta}_{6}\right)$ and $c\left(\hat{\Delta}_{7}\right)$ as shown in Figure 3.19(x). If $d\left(\hat{\Delta}_{7}\right)=3$ then this forces $d\left(\hat{\Delta}_{8}\right)>3$ and $\hat{\Delta}_{9}$ must be given by Figure 3.19(xi) in which case add $c(\Delta)+c\left(\hat{\Delta}_{3}\right) \leq 56$ to $c\left(\hat{\Delta}_{8}\right)$ as shown. Finally if $d\left(\hat{\Delta}_{7}\right)>3$ and $d\left(\hat{\Delta}_{6}\right)=3$ then $d\left(v_{1}\right)<8$ and $l \neq k$ forces $d\left(\hat{\Delta}_{9}\right)>3$ and so $d\left(\hat{\Delta}_{8}\right)=3$ by assumption. If $d\left(v_{5}\right)=5, \geq 7$ then add $c(\Delta)+c\left(\hat{\Delta}_{2}\right) \leq 84, \leq 36$ (respectively) to $c\left(\hat{\Delta}_{7}\right)$ as shown in Figure 3.19(xii).

Let $\Delta$ be given by Figure 3.17(iv) in which case $c(\Delta)>0$ forces $d\left(v_{2}\right)=5$ and $d\left(\hat{\Delta}_{5}\right)>3$. Note also that $k>l$. If $d\left(\hat{\Delta}_{4}\right)>3$ then add $\frac{1}{2}\left(c(\Delta)+c\left(\hat{\Delta}_{1}\right)\right) \leq 22,28$ to each of $c\left(\hat{\Delta}_{4}\right)$ and $c\left(\hat{\Delta}_{5}\right)$ as shown in Figure 3.20(i) according to $d\left(v_{4}\right)=5, d\left(v_{1}\right)=7$ or $d\left(v_{4}\right) \geq 6, d\left(v_{1}\right) \geq 6$. Let $d\left(\hat{\Delta}_{4}\right)=3$. If $\hat{\Delta}_{4}$ is given by Figure 3.20(ii) and $d\left(v_{4}\right)=5, \geq 6$ then add $c(\Delta)+c\left(\hat{\Delta}_{1}\right)=44, \leq 16$ (respectively) to $c\left(\hat{\Delta}_{5}\right)$ as shown. Suppose from now on that $\hat{\Delta}_{4}$ is given by Figure 3.20 (iii). Then $c(\Delta)>0$ forces $d\left(v_{1}\right)=6$. If $d\left(\hat{\Delta}_{8}\right)>3$ and $d\left(\hat{\Delta}_{9}\right)>3$ then add $\frac{1}{2}\left(c(\Delta)+c\left(\hat{\Delta}_{3}\right)\right) \leq 28$ to each of $c\left(\hat{\Delta}_{8}\right)$ and $c\left(\hat{\Delta}_{9}\right)$ as in Figure 3.20(iii); if $d\left(\hat{\Delta}_{8}\right)>3$ and $d\left(\hat{\Delta}_{9}\right)=3$ then add $c(\Delta)+c\left(\hat{\Delta}_{3}\right) \leq 28$ to $c\left(\hat{\Delta}_{8}\right)$ as in (iv); or if $d\left(\hat{\Delta}_{8}\right)=3$ and $d\left(\hat{\Delta}_{9}\right)>3$ then add $c(\Delta)+c\left(\hat{\Delta}_{3}\right) \leq 28, \leq 16$ to $c\left(\hat{\Delta}_{9}\right)$ as in (v), (vi) (respectively) . Let $d\left(\hat{\Delta}_{8}\right)=d\left(\hat{\Delta}_{9}\right)=3$ and suppose first that $\hat{\Delta}_{8}$ is given by Figure 3.20 (vii). If $d\left(\hat{\Delta}_{6}\right)>3$ then add $c(\Delta)+c\left(\hat{\Delta}_{2}\right)=84,=56, \leq 36$ according to $d\left(v_{5}\right)=5,=6$, $\geq 7$ as shown in Figure 3.20(vii) and (viii); or if $d\left(\hat{\Delta}_{6}\right)=3$ then add $c(\Delta)+c\left(\hat{\Delta}_{1}\right)=56, \leq 36$ to $c\left(\hat{\Delta}_{5}\right)$ according to $d\left(v_{4}\right)=6, \geq 7$ as in (ix). Now suppose that $\hat{\Delta}_{8}$ is given by Figure $3.20(\mathrm{x})$. If $d\left(\hat{\Delta}_{6}\right)>3$ then add $c(\Delta)+c\left(\hat{\Delta}_{2}\right)=44, \leq 16$ according to $d\left(v_{5}\right)=5, \geq 6$ as in Figure 3.20(x) and (xi); or if $d\left(\hat{\Delta}_{6}\right)=3$ then add $c(\Delta)+c\left(\hat{\Delta}_{1}\right) \leq 36$ to $c\left(\hat{\Delta}_{5}\right)$ as in (xii).

Let $\Delta$ be given by Figure 3.17(v) and assume that $d\left(v_{i}\right)=3(1 \leq i \leq 3)$, in which case $k>l$ and $d\left(\hat{\Delta}_{4}\right)>3$. If $\hat{\Delta}_{5}$ is given by Figure 3.21(i) or (ii) then when $d\left(v_{4}\right)=5$, add $\frac{1}{3}\left(c(\Delta)+c\left(\hat{\Delta}_{1}\right)\right)=56$ to $c\left(\hat{\Delta}_{4}\right)$ and $\frac{2}{3}\left(c(\Delta)+c\left(\hat{\Delta}_{1}\right)\right)=112$ to $c\left(\hat{\Delta}_{5}\right)$ as shown in (i), or when $d\left(v_{4}\right) \geq 7$ then add $\frac{8}{15}\left(c(\Delta)+c\left(\hat{\Delta}_{1}\right)\right) \leq 64$ to $c\left(\hat{\Delta}_{4}\right)$ and $\frac{7}{15}\left(c(\Delta)+c\left(\hat{\Delta}_{1}\right)\right) \leq 56$ to $c\left(\hat{\Delta}_{5}\right)$ as shown in (ii); if $\hat{\Delta}_{5}$ is given by Figure 3.21(iii) in which it is assumed that $d\left(\hat{\Delta}_{5}\right)>3$ and $d\left(v_{4}\right)=6$ then add $\frac{3}{5}\left(c(\Delta)+c\left(\Delta_{1}\right)\right)=84$ to $c\left(\hat{\Delta}_{4}\right)$ and add $\frac{2}{5}\left(c(\Delta)+c\left(\hat{\Delta}_{1}\right)\right)=56$ to $c\left(\hat{\Delta}_{5}\right)$, or when $d\left(v_{4}\right) \geq 7$ add $\frac{8}{15}, \frac{7}{15}\left(c(\Delta)+c\left(\hat{\Delta}_{1}\right) \leq 64,56\right.$ (respectively) to each of $c\left(\hat{\Delta}_{4}\right)$ and $c\left(\hat{\Delta}_{5}\right)$ as shown. Note that in Figure $3.21(\mathrm{i}), k>l$ forces $d\left(\hat{\Delta}_{6}\right)>3$ and $d\left(\hat{\Delta}_{10}\right)>3$. Assume now that $d\left(\hat{\Delta}_{5}\right)=3$, in which case $d\left(\hat{\Delta}_{6}\right)>3$. If $d\left(\hat{\Delta}_{7}\right)>3$ then add $\frac{1}{3} c(\Delta)+c\left(\hat{\Delta}_{1}\right)=84, \leq 64$ to $c\left(\hat{\Delta}_{4}\right)$ according to $d\left(v_{4}\right)=6$, $\geq 7$ as shown in Figure 3.21(iv) and (v), and when $d\left(v_{5}\right)=5$ add $\frac{2}{5}\left(\frac{2}{3} c(\Delta)+c\left(\hat{\Delta}_{2}\right)\right)=56$ to $c\left(\hat{\Delta}_{6}\right)$ and add $\frac{3}{5}\left(\frac{2}{3} c(\Delta)+c\left(\hat{\Delta}_{2}\right)\right)=84$ to $c\left(\hat{\Delta}_{7}\right)$ as shown in (iv), or when $d\left(v_{5}\right) \geq 6$ then add $\frac{1}{2}\left(\frac{2}{3} c(\Delta)+c\left(\hat{\Delta}_{2}\right)\right) \leq 56$ to each of $c\left(\hat{\Delta}_{6}\right)$ and $c\left(\hat{\Delta}_{7}\right)$ as in (v). Let $d\left(\hat{\Delta}_{7}\right)=3$, in which case $d\left(\hat{\Delta}_{8}\right)>3$. If $d\left(\hat{\Delta}_{9}\right)>3$ then add $\frac{1}{3}\left(c(\Delta)+c\left(\hat{\Delta}_{1}\right)\right)=84, \leq 64$ to $c\left(\hat{\Delta}_{4}\right)$ according to $d\left(v_{4}\right)=6$, $\geq 7$ as shown in Figure 3.21(vi), and when $d\left(v_{6}\right)=5$ add $\frac{3}{5}\left(\frac{2}{3} c(\Delta)+c\left(\hat{\Delta}_{3}\right)\right)=84$ to $c\left(\hat{\Delta}_{8}\right)$ and $\frac{2}{5}\left(\frac{2}{3} c(\Delta)+c\left(\hat{\Delta}_{3}\right)\right)=56$ to $c\left(\hat{\Delta}_{9}\right)$ as in $($ vi $)$, or when $d\left(v_{6}\right) \geq 7$ add $\frac{1}{2}\left(\frac{2}{3} c(\Delta)+c\left(\hat{\Delta}_{3}\right)\right) \leq 46$ to each of $c\left(\hat{\Delta}_{8}\right)$ and $c\left(\hat{\Delta}_{9}\right)$ again as in (vi); or if $d\left(\hat{\Delta}_{9}\right)=3$ then add $\frac{2}{3} c(\Delta)+c\left(\hat{\Delta}_{1}\right)=112,=92$, $\leq 77$ to $c\left(\hat{\Delta}_{4}\right)$ according to $d\left(v_{4}\right)=6,=7, \geq 8$ and add $\frac{1}{3} c(\Delta)+c\left(\hat{\Delta}_{2}\right)=112, \leq 64$ to $c\left(\hat{\Delta}_{6}\right)$ according to $d\left(v_{5}\right)=5, \geq 7$ as shown in (vii) and (viii). Note that $d\left(\hat{\Delta}_{11}\right)>3$ in Figure 3.21(vii).

Let $\Delta$ be given by Figure $3.17(\mathrm{v})$ and assume that $d\left(v_{1}\right) \geq 7$. If $d\left(\hat{\Delta}_{4}\right)>3$ and $d\left(\hat{\Delta}_{5}\right)>3$ then add $\frac{1}{2}\left(c(\Delta)+c\left(\hat{\Delta}_{1}\right)\right) \leq 36$ to each of $c\left(\hat{\Delta}_{4}\right)$ and $c\left(\hat{\Delta}_{5}\right)$ as shown in Figure 3.21(ix); if $d\left(\hat{\Delta}_{4}\right)>3$ and $d\left(\hat{\Delta}_{5}\right)=3$ then add $c(\Delta)+c\left(\hat{\Delta}_{1}\right) \leq 44$ to $c\left(\hat{\Delta}_{4}\right)$ as in (x). Let $d\left(\hat{\Delta}_{4}\right)=3$ in which case $d\left(v_{1}\right) \geq 8$. If $d\left(\hat{\Delta}_{8}\right)>3$ and $d\left(\hat{\Delta}_{9}\right)>3$ add $\frac{1}{2}\left(c(\Delta)+c\left(\hat{\Delta}_{3}\right)\right) \leq 21$ to each of $c\left(\hat{\Delta}_{8}\right)$ and $c\left(\hat{\Delta}_{9}\right)$ as in Figure $3.21(\mathrm{xi})$; if $d\left(\hat{\Delta}_{8}\right)=3$ and $d\left(\hat{\Delta}_{9}\right)>3$ then add $c(\Delta)+c\left(\hat{\Delta}_{3}\right) \leq 42, \leq 14$ to $c\left(\hat{\Delta}_{9}\right)$ according to $d\left(v_{6}\right)=5, \geq 6$ as in (xii); if $d\left(\hat{\Delta}_{8}\right)>3, d\left(\hat{\Delta}_{9}\right)=3$ and $\hat{\Delta}_{9}$ is given by Figure $3.22(\mathrm{i}$ ) 


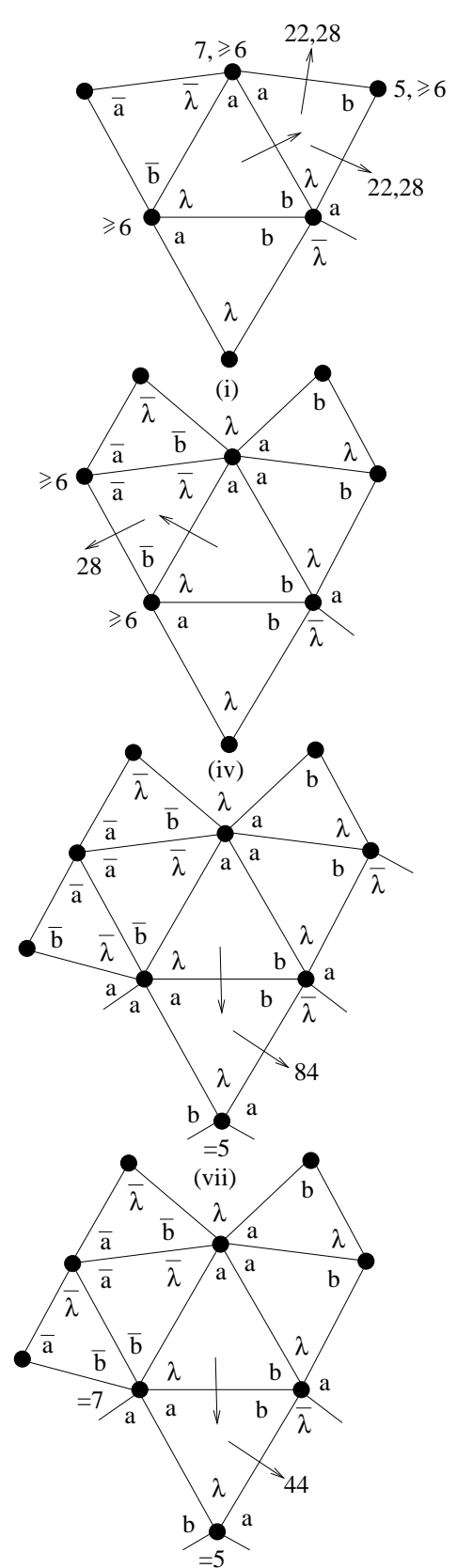

(x)

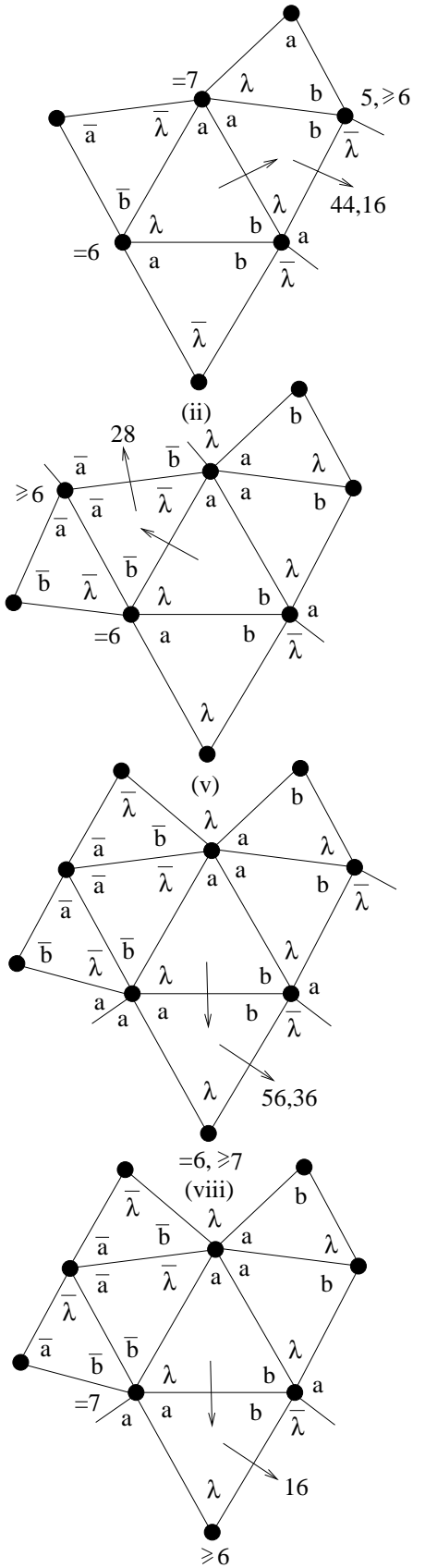

(xi)
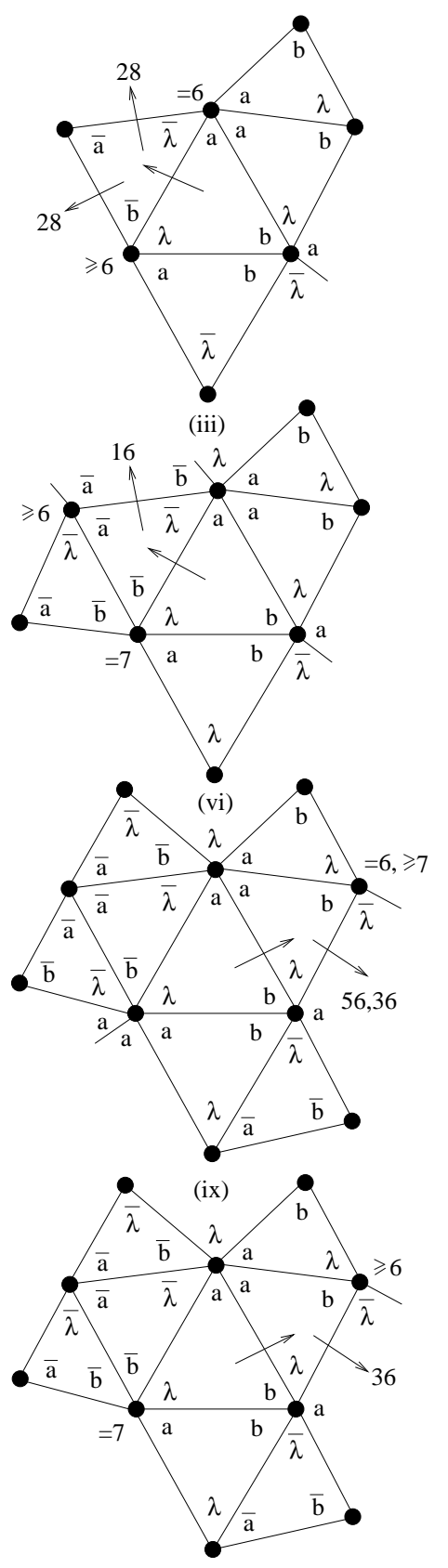

(xii)

Figure 3.20: curvature distribution for $n=7$ 
then add $c(\Delta)+c\left(\hat{\Delta}_{3}\right) \leq 14$ to $c\left(\hat{\Delta}_{8}\right)$ as shown; if $d\left(\hat{\Delta}_{8}\right)>3, d\left(\hat{\Delta}_{9}\right)=3$ and $\hat{\Delta}_{9}$ is given by (ii) then add $c(\Delta)+c\left(\hat{\Delta}_{3}\right) \leq 42$ to $c\left(\hat{\Delta}_{8}\right)$ as shown; if $d\left(\hat{\Delta}_{8}\right)=d\left(\hat{\Delta}_{9}\right)=3$ then $d\left(\hat{\Delta}_{7}\right)>3$ so add $\frac{1}{2}\left(c(\Delta)+c\left(\hat{\Delta}_{2}\right)\right) \leq 105 / 2, \leq 77 / 2$ to each of $c\left(\hat{\Delta}_{8}\right)$ and $c\left(\hat{\Delta}_{7}\right)$ according to $d\left(v_{5}\right)=5, \geq 6$ when $d\left(\hat{\Delta}_{6}\right)>3$ as in (iii); or if $d\left(\hat{\Delta}_{8}\right)=d\left(\hat{\Delta}_{9}\right)=d\left(\hat{\Delta}_{6}\right)=3$ then $d\left(\hat{\Delta}_{5}\right)>3$ so add $c(\Delta)+c\left(\hat{\Delta}_{1}\right) \leq 42$ to $c\left(\hat{\Delta}_{5}\right)$ as in (iv).

Let $\Delta$ be given by Figure $3.17(\mathrm{v})$ and assume that $d\left(v_{2}\right) \geq 7$, in which case $d\left(\hat{\Delta}_{4}\right)>3$ and $k>l$. If $d\left(\hat{\Delta}_{5}\right)>3$ then add $\frac{1}{2}\left(c(\Delta)+c\left(\hat{\Delta}_{1}\right)\right) \leq 36$ to each of $c\left(\hat{\Delta}_{4}\right)$ and $c\left(\hat{\Delta}_{5}\right)$ as shown in Figure $3.22(\mathrm{v})$; or if $\hat{\Delta}_{5}$ is given by Figure $3.22(\mathrm{vi})$ then add $c(\Delta)+c\left(\hat{\Delta}_{1}\right) \leq 36$ to $c\left(\hat{\Delta}_{4}\right)$. Asume then that $\hat{\Delta}_{5}$ is given by Figure 3.22 (vii). If $d\left(\hat{\Delta}_{6}\right)>3$ and $d\left(\hat{\Delta}_{7}\right)>3$ then add $\frac{1}{2}\left(c(\Delta)+c\left(\hat{\Delta}_{2}\right)\right) \leq 36$ to each of $c\left(\hat{\Delta}_{6}\right)$ and $c\left(\hat{\Delta}_{7}\right)$ as in Figure 3.22(vii); if $d\left(\hat{\Delta}_{6}\right)>3, d\left(\hat{\Delta}_{7}\right)=3$ and $d\left(v_{2}\right)=7$ then add $\frac{1}{2} c(\Delta)+c\left(\hat{\Delta}_{1}\right) \leq 54, \leq 6$ to $c\left(\hat{\Delta}_{4}\right)$ according to $d\left(v_{4}\right)=5, \geq 7$ and add $\frac{1}{2} c(\Delta)+c\left(\hat{\Delta}_{2}\right) \leq 54, \leq 6$ to $c\left(\hat{\Delta}_{7}\right)$ according to $d\left(v_{5}\right)=5, \geq 7$ as in (viii); if $d\left(\hat{\Delta}_{6}\right)>3, d\left(\hat{\Delta}_{7}\right)=3$ and $d\left(v_{2}\right) \geq 8$ then add $\frac{1}{2} c(\Delta)+c\left(\hat{\Delta}_{1}\right) \leq 63 / 2$ to $c\left(\hat{\Delta}_{4}\right)$ and add $\frac{1}{2} c(\Delta)+c\left(\hat{\Delta}_{2}\right) \leq 63 / 2$ to $c\left(\hat{\Delta}_{6}\right)$ as in (ix); if $d\left(\hat{\Delta}_{7}\right)>3$ and $\hat{\Delta}_{6}$ is given by $(\mathrm{x})$ then add $\frac{1}{2} c(\Delta)+c\left(\hat{\Delta}_{1}\right) \leq 54, \leq 6$ to $c\left(\hat{\Delta}_{4}\right)$ according to $d\left(v_{4}\right)=5, \geq 7$ and add $\frac{1}{2} c(\Delta)+c\left(\hat{\Delta}_{2}\right) \leq 54, \leq 6$ to $c\left(\hat{\Delta}_{7}\right)$ according to $d\left(v_{5}\right)=5, \geq 7$ as shown; if $d\left(\hat{\Delta}_{7}\right)>3$ and $\hat{\Delta}_{6}$ is given by (xi) then add $\frac{1}{2} c(\Delta)+c\left(\hat{\Delta}_{1}\right) \leq 63 / 2$ to $c\left(\hat{\Delta}_{4}\right)$ and add $\frac{1}{2} c(\Delta)+c\left(\hat{\Delta}_{2}\right) \leq 63 / 2$ to $c\left(\hat{\Delta}_{7}\right)$ as shown; if $d\left(\hat{\Delta}_{6}\right)=d\left(\hat{\Delta}_{7}\right)=3$ then add $c(\Delta)+c\left(\hat{\Delta}_{1}\right) \leq 72, \leq 24, \leq 42$ to $c\left(\hat{\Delta}_{4}\right)$ according to $d\left(v_{4}\right)=5, d\left(v_{2}\right)=7$ or $d\left(v_{4}\right) \geq 7, d\left(v_{2}\right)=7$ or $d\left(v_{4}\right) \geq 5, d\left(v_{2}\right) \geq 8$ as shown in (xii).

Let $\Delta$ be given by Figure 3.17(v) and assume that $d\left(v_{3}\right) \geq 7$, in which case $k>l, d\left(\hat{\Delta}_{4}\right)>3$ and $d\left(\hat{\Delta}_{6}\right)>3$. If $d\left(\hat{\Delta}_{7}\right)>3$ then add $\frac{1}{2}\left(c(\Delta)+c\left(\hat{\Delta}_{2}\right)\right) \leq 36, \leq 21$ to each of $c\left(\hat{\Delta}_{6}\right)$ and $c\left(\hat{\Delta}_{7}\right)$ according to $d\left(v_{6}\right)=7, \geq 8$ as in Figure 3.23(i); if $d\left(\hat{\Delta}_{7}\right)=3$ and $\hat{\Delta}_{7}$ is given by (ii) then add $c(\Delta)+c\left(\hat{\Delta}_{2}\right) \leq 72, \leq 24$ to $c\left(\hat{\Delta}_{6}\right)$ according to $d\left(v_{5}\right)=5, \geq 7$ as shown; or if $d\left(\hat{\Delta}_{7}\right)=3$ and $\hat{\Delta}_{7}$ is given by (iii) then $d\left(v_{5}\right) \geq 6$ and $d\left(v_{6}\right) \geq 8$ so add $c(\Delta)+c\left(\hat{\Delta}_{2}\right) \leq 14$ to $c\left(\hat{\Delta}_{6}\right)$ as shown.

This completes the initial distribution of curvature for $n=7$. Checking Figures 3.16-3.23(i)-(iii) shows that if $d(\hat{\Delta})=3$ then $\hat{\Delta}$ receives positive curvature across at most one edge apart from the eight cases shown in Figure 3.23(iv)-(xi). In Figure 3.24(iv), $\hat{\Delta}$ is (up to inversion) $\hat{\Delta}_{3}$ of Figure 3.18 (ii) and $\hat{\Delta}_{1}$ of Figure 3.19(vii) and add $c\left(\Delta_{1}\right)+c\left(\Delta_{2}\right)+c(\hat{\Delta}) \leq 16$ to $c\left(\hat{\Delta}_{8}\right)$ as shown; in (v) $\hat{\Delta}$ is $\hat{\Delta}_{3}$ of Figure 3.18(ii) and $\hat{\Delta}_{1}$ of Figure 3.19(viii) and add $c\left(\Delta_{1}\right)+c\left(\Delta_{2}\right)+c(\hat{\Delta}) \leq 56,16$ to $c\left(\hat{\Delta}_{8}\right)$ according to $d\left(v_{3}\right)=6,=7$ in $\Delta_{1}$; in (vi) $\hat{\Delta}$ is $\hat{\Delta}_{3}$ of Figure 3.18(iii) and $\hat{\Delta}_{1}$ of Figure 3.20 (ix) and add $c\left(\Delta_{1}\right)+c\left(\Delta_{2}\right)+c(\hat{\Delta}) \leq 84,64,44$ to $c\left(\hat{\Delta}_{8}\right)$ according to $d\left(v_{3}\right)=6,=6,=7$ in $\Delta_{1}$ and $d\left(v_{3}\right)=6,=7, \geq 6$ in $\Delta_{2}$ (respectively) as shown; in (vii) $\hat{\Delta}$ is $\hat{\Delta}_{3}$ of Figure 3.18(v) and $\hat{\Delta}_{3}$ of Figure $3.20(\mathrm{v})$ and add $c\left(\Delta_{1}\right)+c\left(\Delta_{2}\right)+c(\hat{\Delta}) \leq 56$ to $c\left(\hat{\Delta}_{9}\right)$ noting that $d\left(\hat{\Delta}_{9}\right)>3$; in (viii) $\hat{\Delta}$ is $\hat{\Delta}_{1}$ of Figure 3.18(x) and $\hat{\Delta}_{2}$ of Figure 3.19(xii) and add $c\left(\Delta_{1}\right)+c\left(\Delta_{2}\right)+c(\hat{\Delta}) \leq 112,92$ to $c\left(\hat{\Delta}_{5}\right)$ according to $d\left(v_{1}\right)=6,=7$ in $\Delta_{2}$; in (ix) $\hat{\Delta}$ is $\hat{\Delta}_{1}$ of Figure 3.19(vi) and $\hat{\Delta}_{2}$ of $3.20(\mathrm{xi})$ and add $c\left(\Delta_{1}\right)+c\left(\Delta_{2}\right)+c(\hat{\Delta}) \leq 24$ to $c\left(\hat{\Delta}_{5}\right)$; in (x) $\hat{\Delta}$ is $\hat{\Delta}_{1}$ of Figure 3.20(ii) and $\hat{\Delta}_{2}$ of Figure $3.20(\mathrm{x})$ and add $c\left(\Delta_{1}\right)+c\left(\Delta_{2}\right)+c(\hat{\Delta}) \leq 52$ to $c\left(\hat{\Delta}_{5}\right)$; and in (xi) $\hat{\Delta}_{1}$ is $\hat{\Delta}_{1}$ of Figure $3.22(\mathrm{xi})$ and $\hat{\Delta}_{2}$ is $\hat{\Delta}_{2}$ of Figure 3.22(xi) and $\hat{\Delta}_{3}$ of Figure 3.22(ii) and in this case add $c\left(\Delta_{1}\right)+c\left(\hat{\Delta}_{1}\right) \leq 42$ to $c\left(\hat{\Delta}_{4}\right)$ and add $c\left(\Delta_{2}\right)+c\left(\hat{\Delta}_{2}\right) \leq 42$ to $c\left(\hat{\Delta}_{8}\right)$ as shown.

This completes the distribution of curvature scheme for $n=7$. In what follows, unless otherwise stated checking will mean checking Figures 16-23. Indeed checking those figures shows that 84 across an edge is exceeded only in Figures 3.21(i), (vi), (viii) and Figure 3.23(viii), in particular, 112 is the maximum amount to cross an edge. But in Figure 3.21(i) the fact that $k>l$ forces $d\left(\hat{\Delta}_{6}\right)>3$ and $d\left(\hat{\Delta}_{10}\right)>3$; in Figure 3.21 (vii), $k>l$ forces $d\left(\hat{\Delta}_{11}\right)>3$; checking and using in particular the fact that $d\left(v_{4}\right)>5$, that $d\left(\hat{\Delta}_{4}\right)>3$ and $l\left(v_{4}\right)=\lambda^{-1}$ aaw for some sublabel $w$ shows that $\hat{\Delta}_{6}$ receives at most 56 from $\hat{\Delta}_{5}$ in Figures 3.21 (vii) and (viii); and again checking and using $k<l$ shows that $\hat{\Delta}_{5}$ receives at most 56 from $\hat{\Delta}_{6}$ in Figure 3.23(viii), indeed the $(b, \lambda)$-edge of $\hat{\Delta}_{6}$ has length $l$ (as indicated) and this prevents $\hat{\Delta}_{6}$ coinciding with $\hat{\Delta}_{1}$ of Figure $3.18(\mathrm{x}), \hat{\Delta}_{6}$ of Figure 3.19(xii) or $\hat{\Delta}$ of Figure 3.23(viii). 


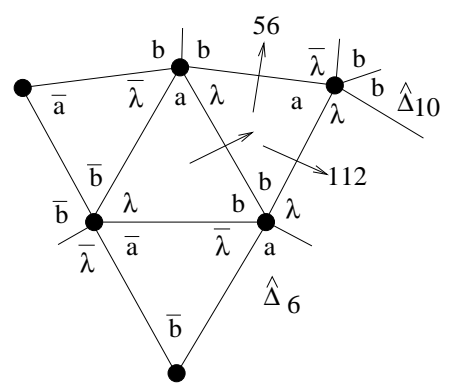

(i)
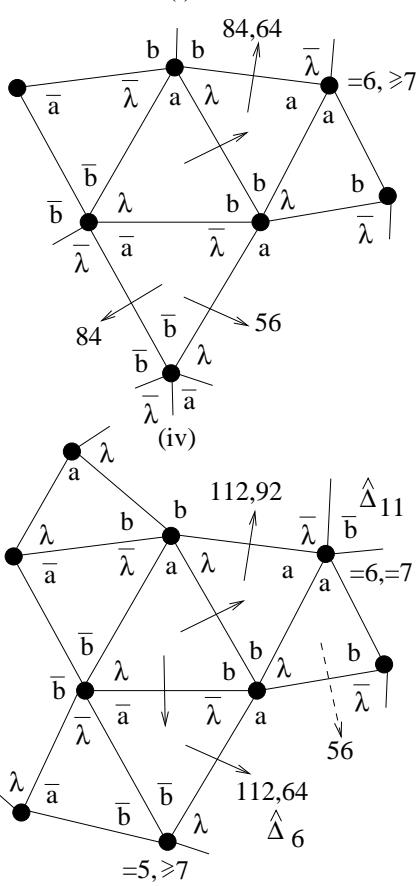

(vii)

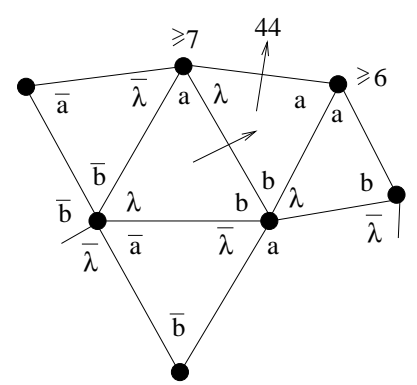

(x)

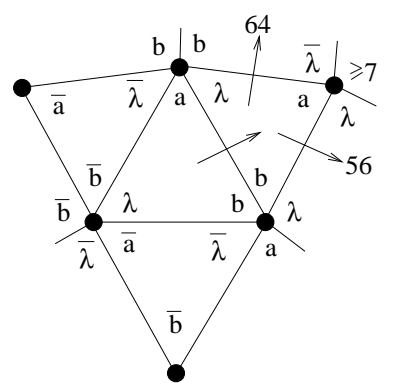

(ii)
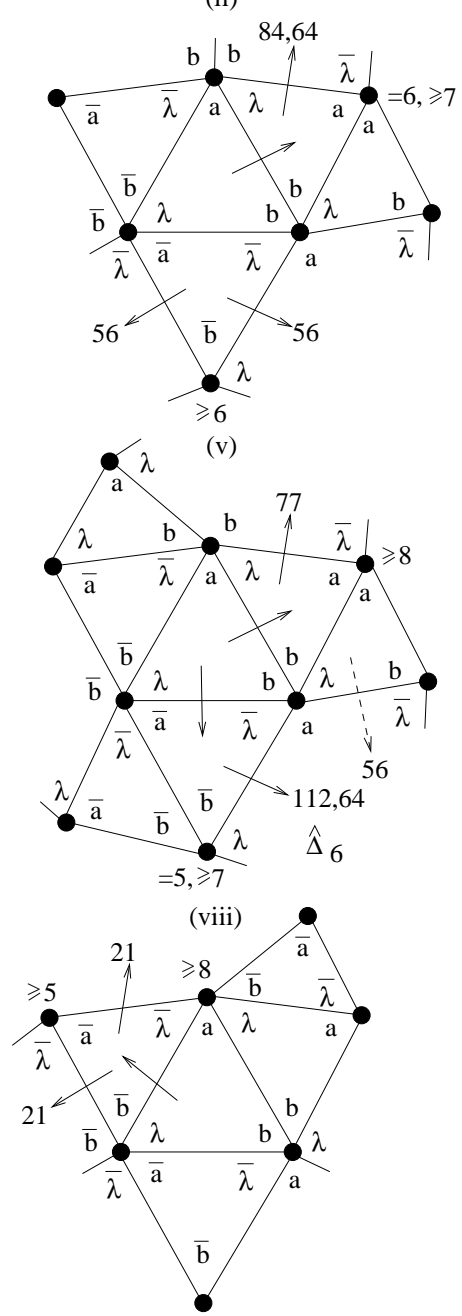

(xi)

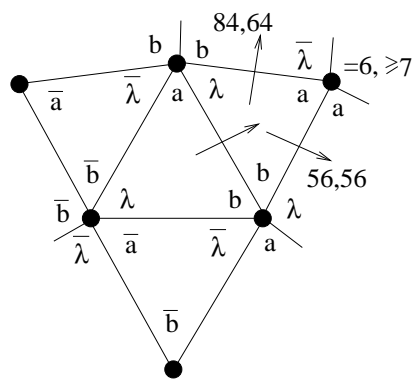

(iii)

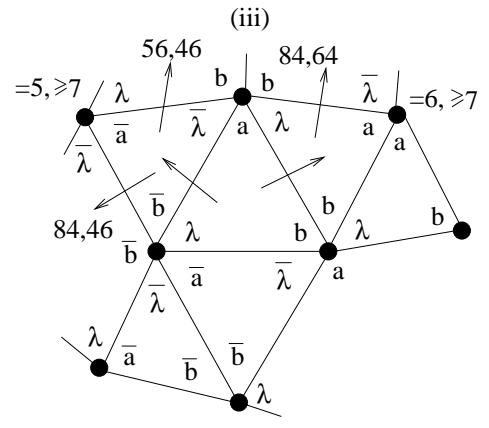

(vi)

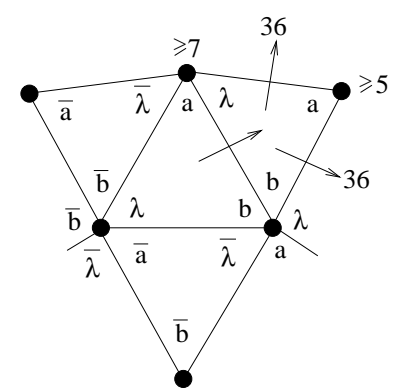

(ix)

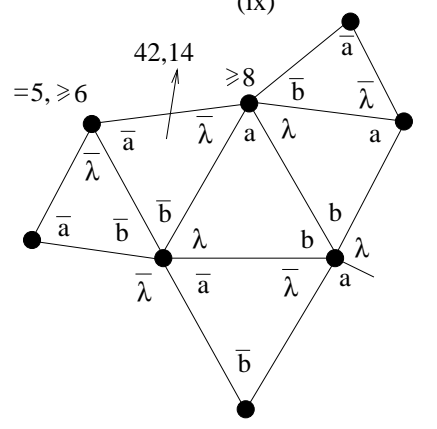

(xii)

Figure 3.21: curvature distribution for $n=7$ 
Let $\hat{\Delta}$ receive positive curvature. If $d(\hat{\Delta})=3$ then it follows from the above that either $c^{*}(\hat{\Delta}) \leq 0$ or the positive curvature is transferred further to a region of degree $>3$. Moreover, it follows from the above paragraph that $c^{*}(\hat{\Delta}) \leq c(\hat{\Delta})+d(\hat{\Delta})(84)$ and so if $d(\hat{\Delta}) \geq 5$ then $c^{*}(\hat{\Delta}) \leq 0$.

Now suppose that $d(\hat{\Delta})=4$. If $\hat{\Delta}$ receives curvature across at most one edge then $c^{*}(\hat{\Delta}) \leq$ $c(5,5,5,5)+112<0$. Suppose that $\hat{\Delta}$ receives curvature across exactly two edges. Since $c(5,5,5,5)+2.84=0$ it can be assumed that $\hat{\Delta}$ receives more than 84 across an edge in which case $\hat{\Delta}$ is $\hat{\Delta}_{4}$ of Figure 3.21(i) and so $\hat{\Delta}$ is given by Figure 2.1(v); or $\hat{\Delta}$ is $\hat{\Delta}_{3}$ of Figure 3.21(vii) or $\hat{\Delta}_{6}$ of Figure 3.21 (vii) or (viii) and so $\hat{\Delta}$ is given by Figure 2.1(vi). (Note that $l \neq 2 k$ forces $d\left(\hat{\Delta}_{5}\right)>4$ in Figure 3.23(viii).) It follows that $\hat{\Delta}$ receives more than 84 across exactly one edge. Now in Figure 3.21(vii), $\hat{\Delta}_{3}$ has a vertex of degree $>5$ and, given $d\left(\hat{\Delta}_{6}\right)=4$, labelling shows that the region $\hat{\Delta}_{6}$ has a vertex of degree $>5$ in Figures 3.21(vii) and (viii); so for these cases $c^{*}(\hat{\Delta}) \leq c(5,5,5,6)+112+84=0$. Moreover, if $\hat{\Delta}$ is $\hat{\Delta}_{4}$ of Figure 3.21(i) and each vertex of $\hat{\Delta}$ has degree 5 then $\hat{\Delta}$ is given by Figure 3.24(i); but checking shows that $\hat{\Delta}$ receives at most 56 from the region $\Delta$ and so $c^{*}(\hat{\Delta}) \leq c(5,5,5,5)+112+56=0$. Therefore if $\hat{\Delta}$ receives positive curvature across exactly two edges then $c^{*}(\hat{\Delta}) \leq 0$.

Let $\hat{\Delta}$ be given by Figure 2.1(v) and assume that $\hat{\Delta}$ receives positive curvature across at least three edges. Since $d\left(\hat{\Delta}_{6}\right)>3$ and $d\left(\hat{\Delta}_{10}\right)>3$ in Figure 3.21(i) it follows that the maximum curvature $\hat{\Delta}$ receives across an edge is 56 or 84 . Furthermore, checking shows that if $e$ is an edge of $\hat{\Delta}$ having at least one endpoint of degree $>5$ then $\hat{\Delta}$ receives at most 56 across $e$.

Suppose that all four neighbouring regions of $\hat{\Delta}$ have degree 3 so by Figure 3.12 there are 24 possibilities. Using the same notation as for $n=8$ if $(1,3,5,8)$ or $(2,3,5,8)$ then $l=2 k$. It follows from the above remarks that $\hat{\Delta}$ receives at most 4.56 in the following 18 cases: $(1,3,5,9),(6,6,5,6)$ (since $l>k$ prevents $\left.d_{2}=5\right),(224,252) ;(1,3,6,8),(6,5,7,6),(224,272) ;(1,3,6,9),(6,5,7,5)$, $(224,244) ;(1,3,7,8),(6,5,6,7),(224,272) ;(1,3,7,9),(6,5,6,7),(224,272) ;(1,4,5,8),(6,6,6,5)$, $(224,256) ;(1,4,5,9),(6,6,6,6),(224,280) ;(1,4,6,8),(6,6,7,6),(224,300) ;(1,4,6,9),(6,6,7,5)$, $(224,272) ;(1,4,7,8),(6,6,5,7),(224,272) ;(1,4,7,9),(6,6,5,7),(224,272) ;(2,3,5,9)(l>k)$, $(5,7,5,6),(224,244) ;(2,3,6,8),(6,7,7,6)(224,320) ;(2,3,7,8),(6,7,6,7),(224,320) ;(2,3,7,9)$, $(5,7,6,7),(224,292) ;(2,4,5,8),(6,5,6,5),(224,224) ;(2,4,5,9),(5,5,6,6),(224,224) ;$ and $(2,4,6,8),(6,5,7,6),(224,272)$.

For $(2,3,6,9)$ we have $(5,7,7,5),(56,56,56,84),(252,264)$. For $(2,4,6,9),\left(d_{1}, d_{2}, d_{3}, d_{4}\right)=$ $(5,5,7,5)$ and $\left(m_{1}, m_{2}, m_{3}, m_{4}\right)=(56,56,56,84)$ so if $d_{i} \neq 5$ for any $i \in\{1,2,4\}$ then $(\Sigma, \tilde{c})=$ $(252,264)$. Assume otherwise, in which case $\hat{\Delta}$ is given by Figure 3.24(ii) in which $d\left(\Delta_{10}\right)>3$ and checking shows that $m_{3}=42$ (see Figure 3.21(xii)). If $\left.d(\Delta)_{11}\right)>3$ in Figure 3.24(ii) then $\left(m_{1}, m_{2}, m_{3}, m_{4}\right)=(28,56,42,84)$ and $(\Sigma, \tilde{c})=(210,216)$; or if $d\left(\Delta_{11}=3\right.$ then $d\left(\Delta_{12}>3\right.$ and again checking shows that $m_{2}=28$ therefore $\left(m_{1}, m_{2}, m_{3}, m_{4}\right)=(56,28,42,84)$ and $(\Sigma, \tilde{c})=$ $(210,216)$. For $(2,4,7,8),\left(d_{1}, d_{2}, d_{3}, d_{4}\right)=(6,5,5,7)$ and if $d_{j} \neq 5$ for $j=2$ or 3 then $d_{j} \geq 7$ and $(\Sigma, \tilde{c})=(224,292)$. Assume otherwise, in which case $\hat{\Delta}$ is given by Figure 3.24(iii) in which $d\left(\Delta_{12}\right)=3$ forces $k \leq l$ and $d\left(\Delta_{13}\right)=3$ forces $k>l$ and it follows that $m_{2}=56$ and $(\Sigma, \tilde{c})=$ $(224,224)$. For $(2,4,7,9),\left(d_{1}, d_{2}, d_{3}, d_{4}\right)=(5,5,5,7)$ and $\left(m_{1}, m_{2}, m_{3}, m_{4}\right)=(56,84,56,56)$ so if $d_{i} \neq 5$ for any $1 \leq i \leq 3$ then $d_{i} \geq 7$ and $(\Sigma, \tilde{c})=(252,265)$. Assume otherwise, so $\hat{\Delta}$ is given by Figure 3.24(iv) in which $d\left(\Delta_{10}\right)>3$, moreover $d\left(\Delta_{11}\right)=d\left(\Delta_{12}\right)=3$ contradicts $l \neq k$ and checking shows that $m_{3}=36$, therefore if $d\left(\Delta_{11}\right)>3$ then $\left(m_{1}, m_{2}, m_{3}, m_{4}\right)=(28,84,36,56)$ and $(\Sigma, \tilde{c})=(204,216)$ or if $d\left(\Delta_{11}\right)>3$ then $\left(m_{1}, m_{2}, m_{3}, m_{4}\right)=(56,56,36,56)$ and $(\Sigma, \tilde{c})=$ $(204,216)$.

Now suppose that exactly three of the neighbouring regions of $\hat{\Delta}$ are 3 -gons. There are 44 possibilities as shown in Figure 3.12. For $(1,3,5)$ the fact that $k<l$ forces $d_{2} \geq 7$ and we have $(6,7,5,5),(56,56,84,-)$ and $(\Sigma, \tilde{c})=(196,244)$. Observe that for $(2,4,7),(2,4,9)$ and $(2,6,5) \Delta_{2}$ of Figure 3.12 cannot be $\Delta$ of Figure 3.16(i) and so for $(2,4,7)$ we have $(5,5,5,7),(56,84,56,-),(196,216)$; for $(2,4,9)$ labelling prevents $d_{3}=d_{4}=5$ so either $(5,5,5,7),(56,84,-, 56)$ or $(5,5,7,5),(56,56,-, 84)$ occurs and $(\Sigma, \tilde{c})=(196,216)$; and for 

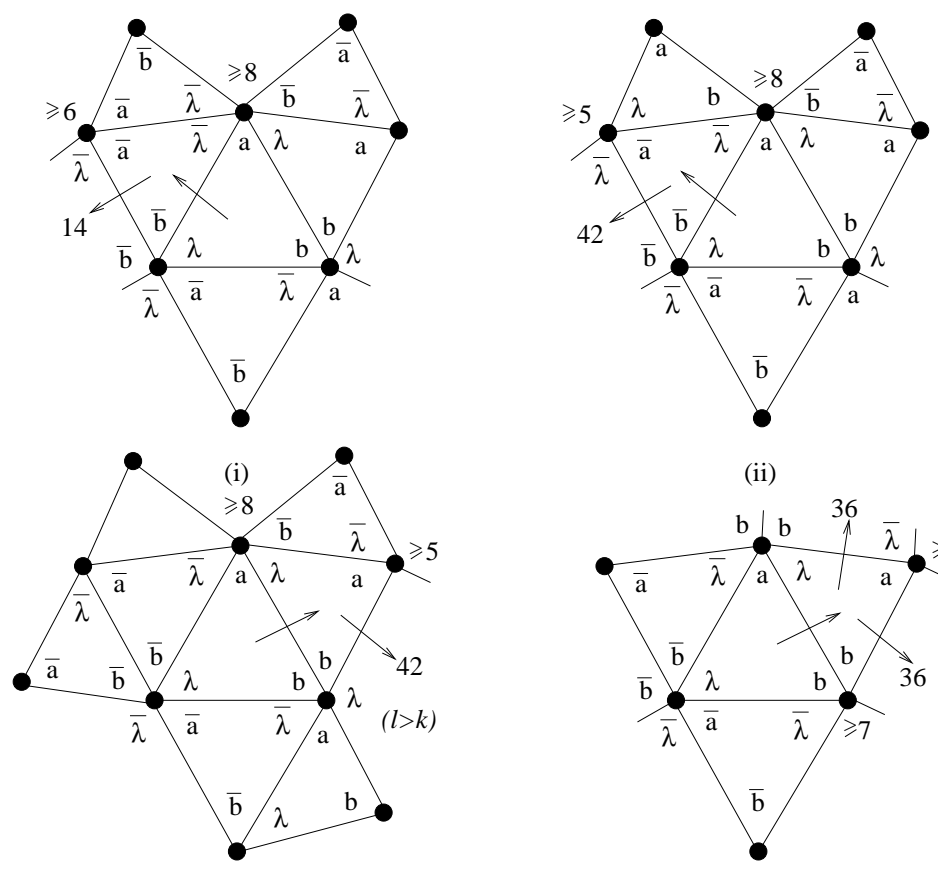

(ii)
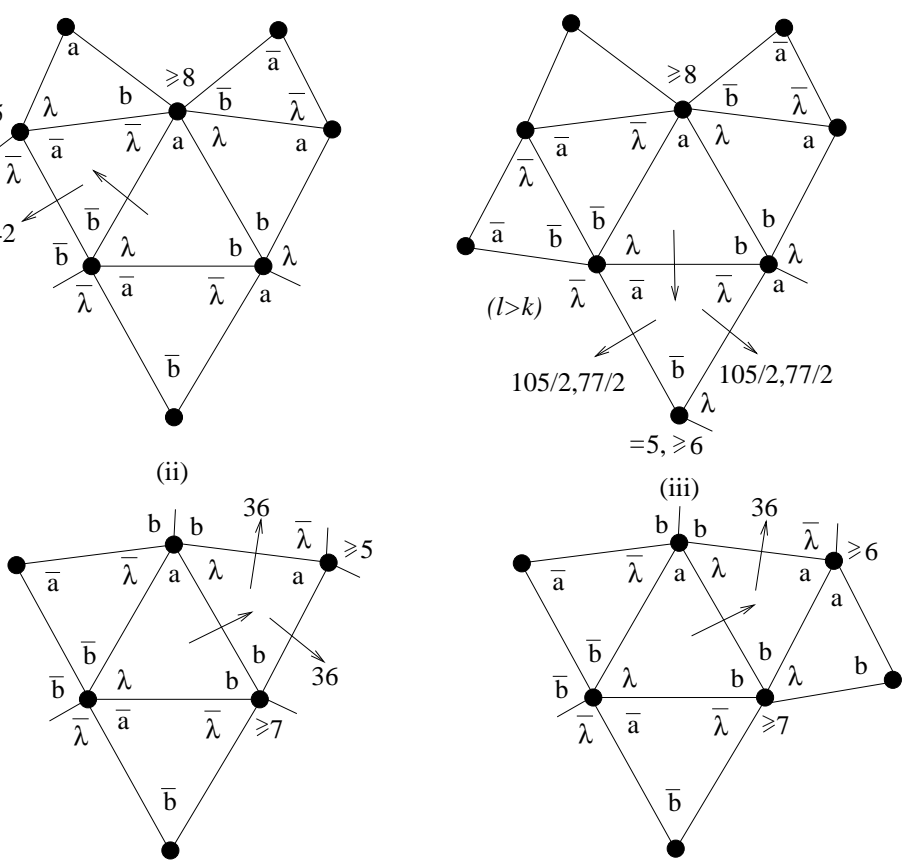

(iv)

(v)

(vi)
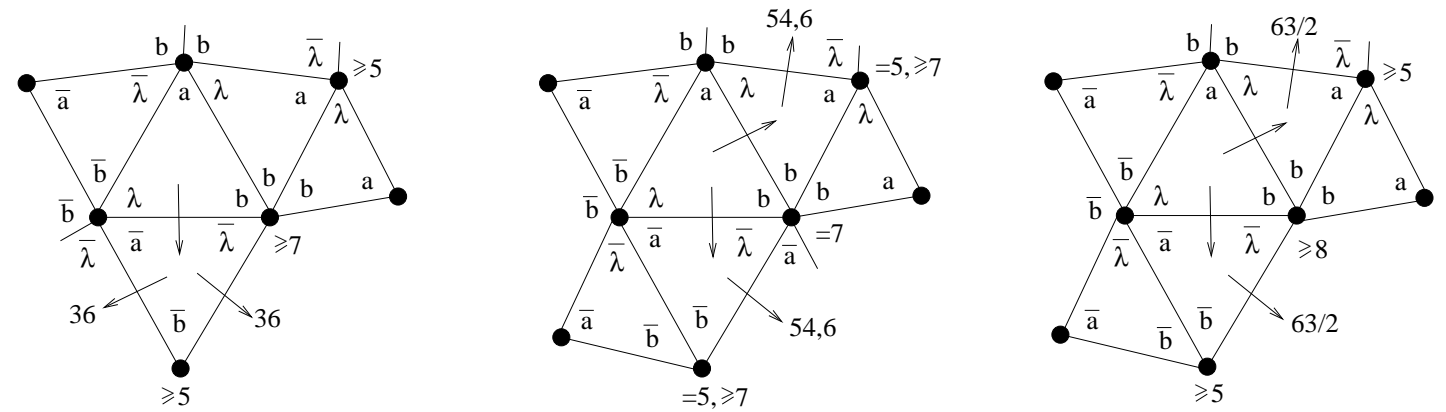

(vii)

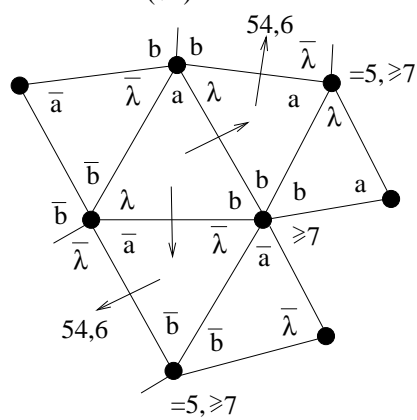

(x)

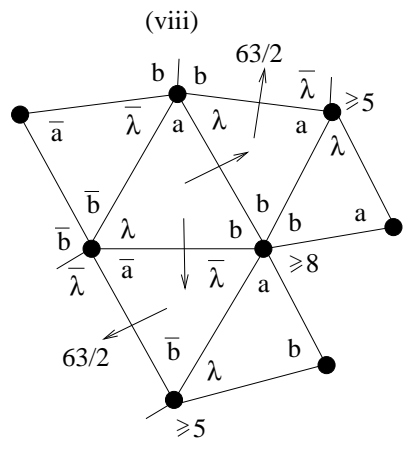

(xi)

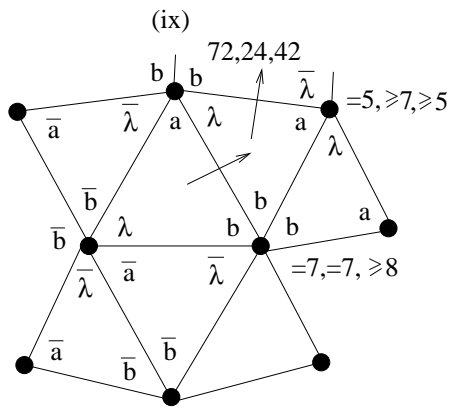

(xii)

Figure 3.22: curvature distribution for $n=7$ 


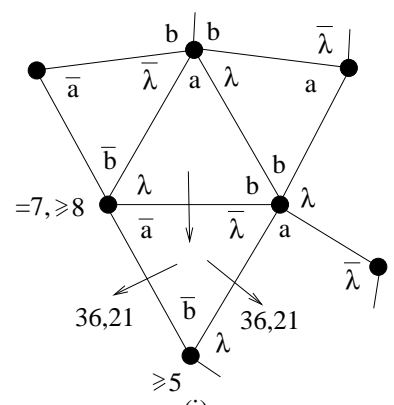

(i)

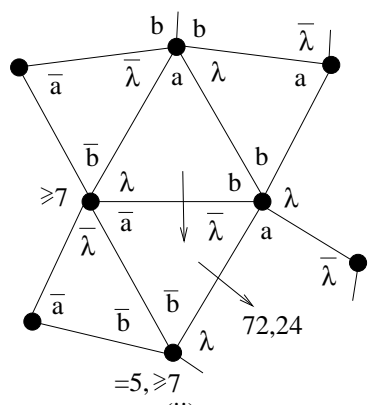

(ii)

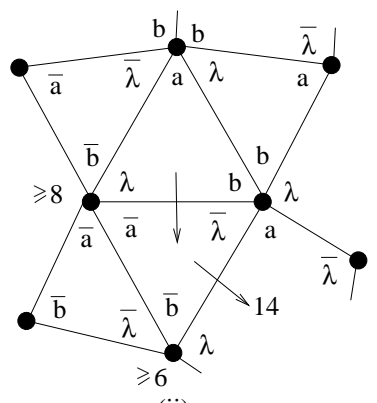

(ii)

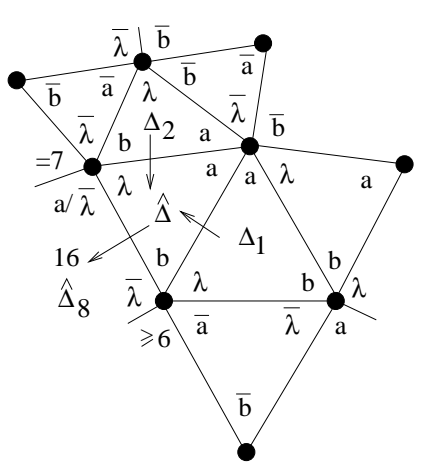

(iv)

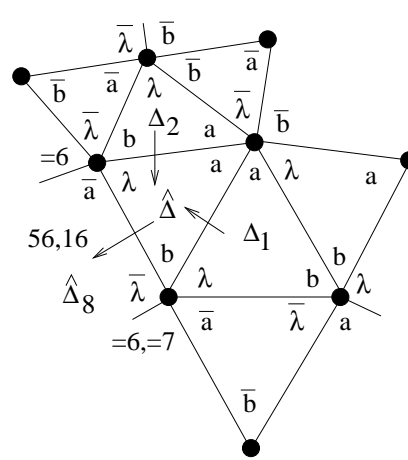

(v)

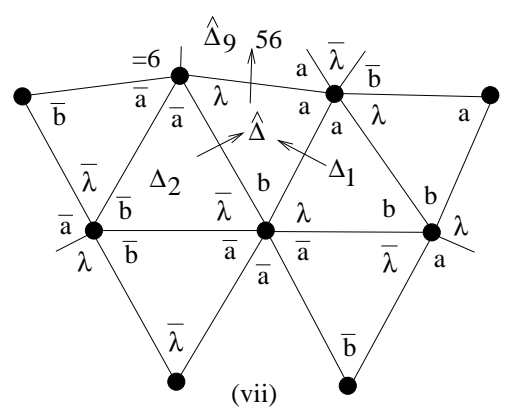

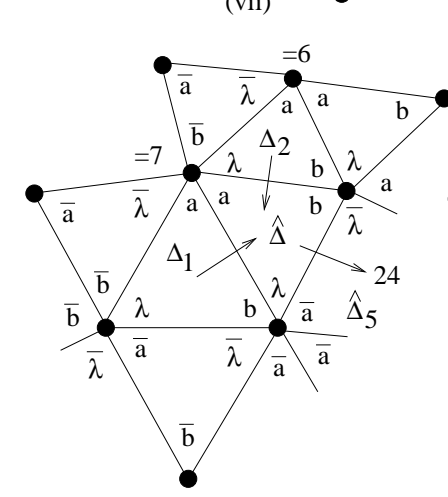

(ix)

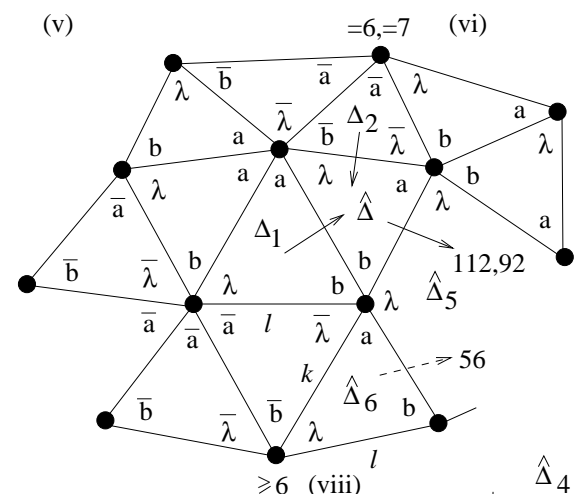

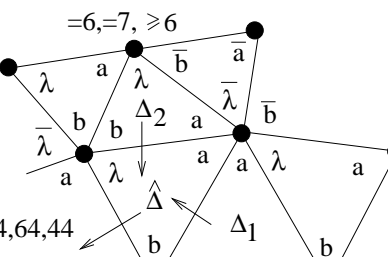

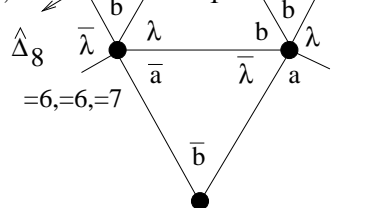

(vi)

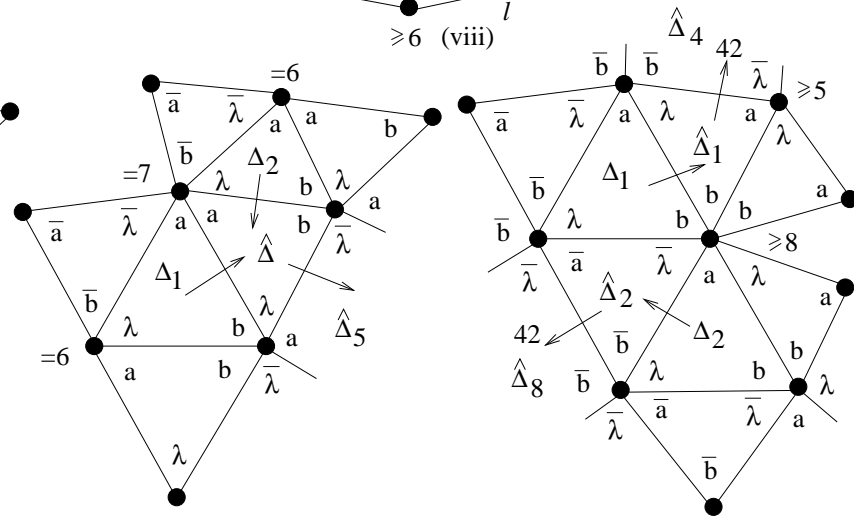

(x)

(xi)

Figure 3.23: curvature distribution for $n=7$ 

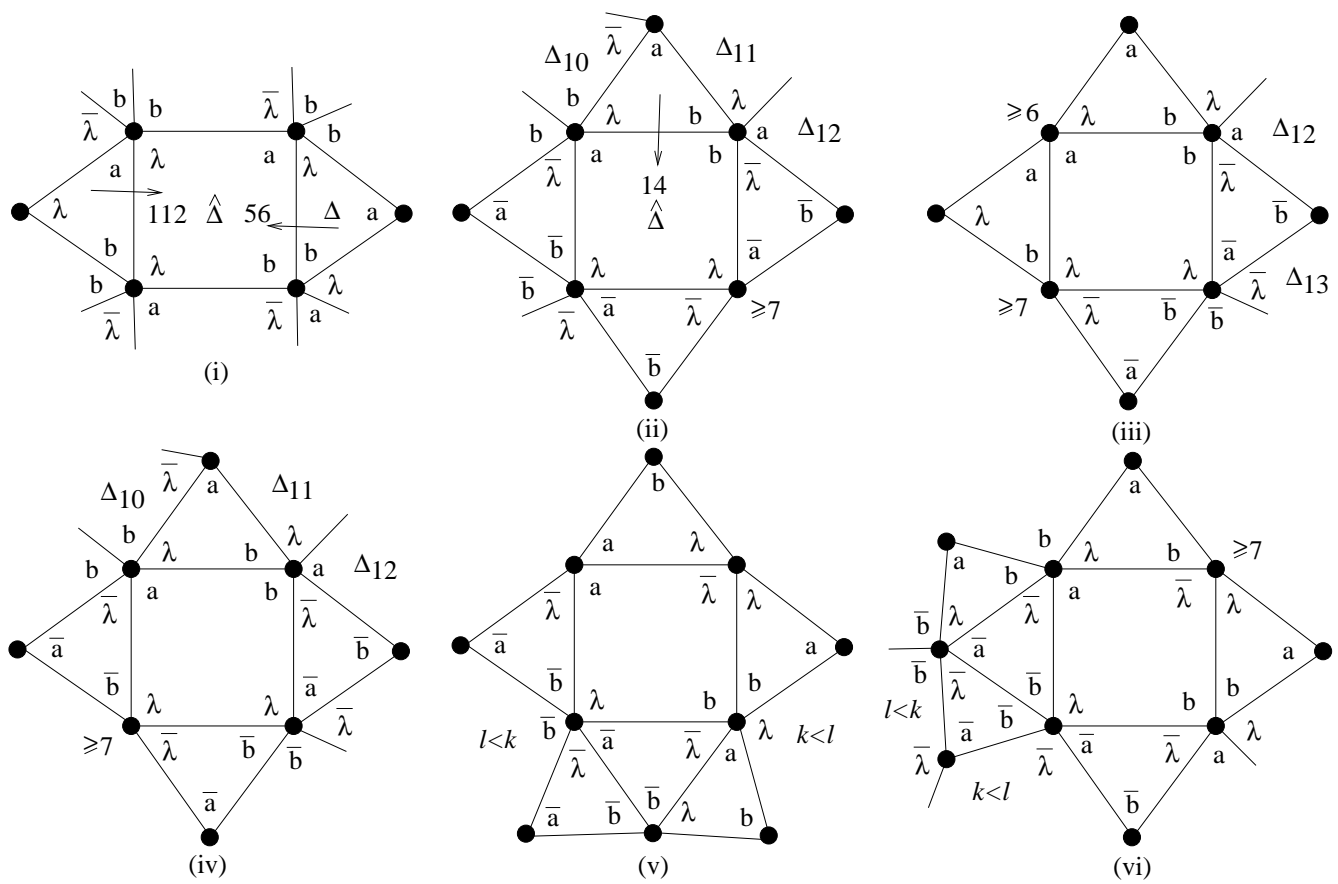

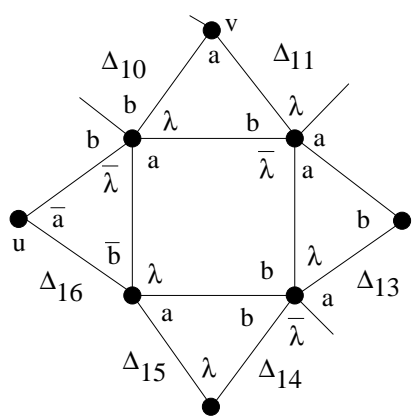

(vii)

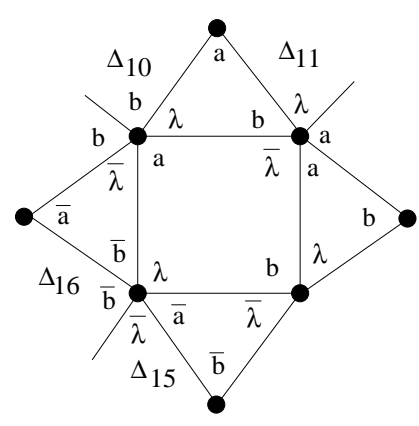

(viii)

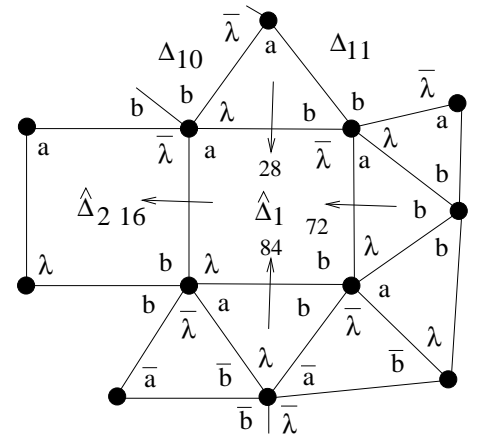

(ix)

Figure 3.24: degree 3 neighbours of a degree 4 region for $\mathrm{n}=7$ 
$(2,6,9)$ we have $(5,5,7,5),(56,-, 56,84),(196,216)$. For $(3,5,8)$ we have $l=2 k$, a contradiction. In the remaining 39 cases two of the neighbouring 3 -gons share a vertex of degree $\geq 6$ with $\hat{\Delta}$ and it follows that $c^{*}(\hat{\Delta}) \leq c(5,5,5,6)+2.56+84=0$.

Let $\hat{\Delta}$ be given by Figure 2.1(vi) and suppose that $\hat{\Delta}$ receives positive curvature across four edges and so there are 16 possibilities as can be seen in Figure 3.13. Let $e$ be an edge of $\hat{\Delta}$. If both endpoints of $e$ have degree greater than 5 then the maximum curvature across $e$ is 56 ; or if exactly one endpoint of $e$ has degree 5 then the maximum is 56 or $e$ occurs in Figure 3.21 or 3.23. Given this, checking yields the following: $(1,3,5,7),(6,7,7,6),(56,56,56,56),(224,320) ;(1,3,5,8)$ forces $k=l$, a contradiction; $(1,3,6,8),(6,7,5,6),(56,56,56,56),(224,272) ;(1,4,5,7),(6,7,5,6)$, $(56,92,56,56),(260,272) ;(1,4,5,8)$ forces $k=l$, a contradiction; $(1,4,6,7),(6,7,6,5),(56,56,56,56)$, $(224,272) ;(1,4,6,8),(6,7,6,6),(56,56,56,56),(224,300) ;(2,3,5,7),(5,7,7,6),(56,56,56,64)$, $(232,292) ;(2,3,5,8)$ forces $k=l$, a contradiction; $(2,3,6,8),(6,7,5,6),(56,56,56,56),(224,272)$; $(2,4,5,8)$ forces $k=l$, a contradiction; and $(2,4,6,8),(6,5,6,6),(56,56,56,56),(224,252)$. This leaves $(1,3,6,7),(2,3,6,7),(2,4,5,7)$ and $(2,4,6,7)$ to consider.

For $(1,3,6,7),(6,7,5,5)$ and $(56,56,84,56)$ hold so if either $d_{3}>5$ or $d_{4}>5$ then $(\Sigma, \tilde{c})=$ $(252,292)$; if $d_{3}=d_{4}=5$ and $m_{3}=56$ then $(\Sigma, \tilde{c})=(224,244)$; or if $d_{3}=d_{4}=5$ and $m_{3}>56$ then (see Figure 3.16(iii)) $\hat{\Delta}$ is given by Figure 3.24(v), but, as shown, the labelling forces both $k>l$ and $k<l$, a contradiction.

For $(2,3,6,7)$ we have $(5,7,5,5)$ and $(56,56,56,84)$ noting that $m_{3}=56$ by the argument for $(1,3,6,7)$. If $d_{i}>5$ for any $i \in\{1,3,4\}$ then $d_{i} \geq 7$ and $(\Sigma, \tilde{c})=(252,264)$, so assume otherwise. If $m_{4}>56$ then $\hat{\Delta}$ is given by Figure 3.24(vi) but, as shown, the labelling forces both $k>l$ and $l>k$, a contradiction; so if $d_{2} \geq 8$ then $(\Sigma, \tilde{c})=(224,231)$. On the other hand if $d_{2}=7$ then checking shows that $m_{2}=36$ and so $(\Sigma, \tilde{c})=(204,216)$.

For $(2,4,5,7)$ we have $(5,5,5,6)$ and $(56,77,56,64)$ (see Figure 3.21 (viii)). If at least two $d_{i}>5$ for $1 \leq i \leq 3$ then $d_{i} \geq 7$ and $(\Sigma, \tilde{c})=(253,292)$; if only $d_{1}>5$ then we have $(7,5,5,6)$, $(56,72,56,56)$ (see Figure $3.22(x i i))$ and $(\Sigma, \tilde{c})=(240,244)$; if only $d_{3}>5$ then $(5,5,7,6)$, $(56,56,56,64)$ and $(\Sigma, \tilde{c})=(232,244)$, if only $d_{2}>5$ and $d_{2}=7$ then $(5,7,5,6),(56,64,56,64)$ and $(\Sigma, \tilde{c})=(240,244)$ or if $d_{2} \geq 8$ then $(5,8,5,6),(56,77,56,64)$ and $(\Sigma, \tilde{c})=(253,259)$. Assume then that $d_{i}=5(1 \leq i \leq 3)$ in which case $\hat{\Delta}$ is given by Figure 3.24 (vii) where $k>l$ and $d\left(\Delta_{11}\right)>3$. If $d\left(\Delta_{10}\right)=3$ then $d(v) \geq 6$ and $m_{1}=28$ so if $d_{4}=6$ then $(28,56,56,56)$ and $(\Sigma, \tilde{c})=(196,196)$ or if $d_{4} \geq 7$ then $(28,56,56,64)$ and $(\Sigma, \tilde{c})=(204,216)$. So let $d(\Delta)_{10}>3$ in Figure 3.24 (vii) in which case $\hat{\Delta}$ cannot be $\hat{\Delta}_{6}$ of Figure 3.21 (vii) or (viii) and so $m_{4}=56$. It follows that if $d_{4} \geq 7$ then we have $(28,72,56,56)$ and $(\Sigma, \tilde{c})=(212,216)$; or if $d_{4}=6$ then in fact either $d\left(\Delta_{16}\right)>3$ or $d\left(\Delta_{16}\right)=3$ but $d(u) \geq 6$ and in both cases we have $(28,72,56,28)$ and $(\Sigma, \tilde{c})=(184,196)$.

For $(2,4,6,7)$ we have $(5,5,6,5)$ and $(56,56,56,64)$ since $m_{4}=64$ by the argument for $(2,3,6,7)$. If any of $d_{i}>5$ for $i \in\{1,2,4\}$ then $d_{i} \geq 7$ and $(\Sigma, \tilde{c})=(232,244)$, so assume otherwise in which case $\hat{\Delta}$ is given by Figure 3.24(viii). If $k>l$ then $d\left(\Delta_{11}\right)>3$ in Figure 3.24(viii), forcing $(28,56,56,56)$ and $(\Sigma, \tilde{c})=(196,196)$; or if $k<l$ then $d\left(\Delta_{10}\right)>3$ and then either $d\left(\Delta_{15}\right)>3$ in which case we have $(42,56,42,56)$ or $d\left(\Delta_{15}\right)=3$ forcing $d\left(\Delta_{16}\right)>3$ and $(42,56,56,42)$ and in both cases $(\Sigma, \tilde{c})=(196,196)$.

Suppose now that exactly three of the neighbouring regions of $\hat{\Delta}$ are 3 -gons, and so, by Figure 3.13 there are 32 possibilities. If $(1,5,8),(2,5,8),(3,5,8)$ or $(4,5,8)$ occurs then $l=k$, a contradiction. For $(1,4,5)$ we have $(6,7,5,5),(56,84,84,-)$ and $(\Sigma, \tilde{c})=(224,224)$. For $(2,4,5)$ we have $(5,5,5,5)$ and $(56,77,84,-)$ so if at least two vertices have degree $\geq 6$ then $(\Sigma, \tilde{c})=$ $(217,224)$; if $d_{1}>5$ only this yields $(7,5,5,5),(56,72,84,-)$ and $(\Sigma, \tilde{c})=(212,216)$; if $d_{2}>5$ only and $d_{2}=7$ then $(56,64,84,-)$ and $(\Sigma, \tilde{c})=(204,216)$ or $d_{2} \geq 8$ then $(56,77,84,-)$ and $(\Sigma, \tilde{c})=(217,231)$; if $d_{3}>5$ only then $d_{3} \geq 7,(56,77,56,-)$ and $(\Sigma, \tilde{c})=(189,216)$; if $d_{4}>5$ only then $d_{4} \geq 6,(56,77,56,-)$ and $(\Sigma, \tilde{c})=(189,196)$; if $d_{i}=5(1 \leq i \leq 4)$ then 
(see $(2,4,5,7)) k>l$ and $m_{1}=28$ so if $m_{2}=56$ then $(\Sigma, \tilde{c})=(168,168)$ or if $m_{3}=56$ then $(\Sigma, \tilde{c})=(156,168)$. It can be assumed therefore that $\hat{\Delta}$ is given by $\hat{\Delta}_{1}$ of Figure $3.24(\mathrm{ix})$ in which $d\left(\Delta_{10}\right)>3, d\left(\Delta_{11}\right)>3, d\left(\hat{\Delta}_{2}\right)>3$ and $(\Sigma, \tilde{c})=(184,168)$. In this case we have the extra distribution rule: add $c^{*}\left(\hat{\Delta}_{1}\right) \leq 16$ to $\hat{\Delta}_{2}$ as shown. For $(1,4,8)$ we have $(6,7,5,5)$, $(56,77,-, 60)$ and $(\Sigma, \tilde{c})=(193,244)$. For $(2,3,7)$ we have $(5,7,5,5)$ and $(56,56,-, 112)$ so if at least two vertices have degree $\geq 6$ then $(\Sigma, \tilde{c})=(224,224)$; or if $d_{i}=5$ for $i \in\{1,3,4\}$ then checking shows that $m_{2}=36$ and $(\Sigma, \tilde{c})=(204,216)$. For $(2,4,7)$ labelling forces $(5,5,5 / 6,6 / 5)$ and $(56,72,-, 112)$ so if $d_{1}>5$ or $d_{2}>5$ this yields $(7 / 5,5 / 7,5 / 6,6 / 5)$ and $(\Sigma, \tilde{c})=(240,244)$; if $d_{i}=5(1 \leq i \leq 3)$ then $d_{4} \geq 6, m_{4}=64$ and $(\Sigma, \tilde{c})=(192,196)$; if $d_{i}=5$ for $i \in\{1,2,4\}$ and $d_{3} \geq 7$ then checking shows that $m_{2}=42$ (see Figure $\left.3.22(\mathrm{ii})\right)$ and $(\Sigma, \tilde{c})=(210,216)$; if $d_{i}=5$ for $i \in\{1,2,4\}$ and $d_{3}=6$ then either $m_{4}=84$ and $(\Sigma, \tilde{c})=(196,196)$ or $m_{4}=112, k>l$ forcing $m_{1}=28($ see $(2,4,5,7))$ and again $(\Sigma, \tilde{c})=(196,196)$; or if $d_{1}=d_{2}=5$ only then $(5,5,6,6)$, $(56,56,-, 64)$ and $(\Sigma, \tilde{c})=(176,224)$. For $(2,4,8)$ labelling forces $(6,5,5 / 6,6 / 5),(56,84,-, 56)$ and $(\Sigma, \tilde{c})=(196,224)$. For $(2,5,7)$ we have $(5,5,5,6)$ and $(84,-, 56,64)$ so if at least two vertices have degree $\geq 6$ then $(\Sigma, \tilde{c})=(204,224)$; if $d_{4} \geq 7$ then $(\Sigma, \tilde{c})=(204,216)$; or if $d_{4}=6$ then $m_{4}=56$ and $(\Sigma, \tilde{c})=(196,196)$. For $(2,6,7)$ labelling forces $(5,5 / 7,6 / 5,5)$ and $(84,-, 84,64)$ (see $(2,4,6,7)$ for $\left.m_{4}=64\right)$ so if $d_{1}>5$ or $d_{4}>5$ then $(\Sigma, \tilde{c})=(232,244)$; if $d_{1}=d_{4}=5$, $d_{2} \geq 6$ and $d_{3} \geq 6$ this yields $(84,-, 84,56)$ and $(\Sigma, \tilde{c})=(224,224)$; if $d_{i}=5$ for $i \in\{1,2,4\}$ then $(84,-, 56,56)$ and $(\Sigma, \tilde{c})=(196,196)$; or if $d_{i}=5$ for $i \in\{1,3,4\}$ then $(56,-, 84,56)$ and $(\Sigma, \tilde{c})=(196,216)$. For $(3,6,7)$ we have $(5,7,5,5)$ and $(-, 56,84,64)$ (see $(2,4,6,7)$ for $\left.m_{4}=64\right)$ and so $(\Sigma, \tilde{c})=(204,216)$. For $(4,5,7)$ we have $(5,5,5,6)$ and $(-, 112,56,56)$ so if at least two vertices have degree $\geq 6$ then $(\Sigma, \tilde{c})=(224,224)$; or if $d_{i}=5(1 \leq i \leq 3)$ this yields $(-, 72,56,56)$ and $(\Sigma, \tilde{c})=(184,196)$. For the 18 cases that remain, checking shows that $\hat{\Delta}$ receives at most $84+2.56$ across three edges and contains at least one vertex of degree $\geq 6$ and so $(\Sigma, \tilde{c})=(196,196)$.

To complete the case $n=7$ it remains to consider $\hat{\Delta}=\hat{\Delta}_{2}$ of Figure 3.24(ix). If $d(\hat{\Delta})>4$ then exactly as before, $c^{*}(\hat{\Delta}) \leq 0$ so assume $d(\hat{\Delta})=4$ as shown. If $\hat{\Delta}$ receives curvature across at most two edges then $c^{*}(\hat{\Delta}) \leq c(5,5,5,5)+16+112<0$. If $\hat{\Delta}$ receives across more than two edges then we see from Figure 3.13 that the possibilities are $\left(2, \hat{\Delta}_{1}, 5\right),\left(2, \hat{\Delta}_{1}, 7\right),\left(2, \hat{\Delta}_{1}, 8\right),\left(\hat{\Delta}_{1}, 5,7\right)$, $\left(\hat{\Delta}_{1}, 5,8\right),\left(2, \hat{\Delta}_{1}, 5,7\right)$ and $\left(2, \hat{\Delta}_{1}, 5,8\right)$. If $\left(\hat{\Delta}_{1}, 5,8\right)$ or $\left(2, \hat{\Delta}_{1}, 5,8\right)$ then $l=k$, a contradiction; if $\left(2, \hat{\Delta}_{1}, 5\right)$ then we have $(56,18,84,-)$ and $(\Sigma, \tilde{c})=(156,168)$; if $\left(2, \hat{\Delta}_{1}, 7\right)$ then $d_{4} \geq 6$ which yields $(56,16,-, 64)$ and $(\Sigma, \tilde{c})=(136,196)$; if $\left(2, \hat{\Delta}_{1}, 8\right)$ then $d_{1} \geq 6$ which yields $(56,16,-, 56)$ and $(\Sigma, \tilde{c})=(128,196)$; if $\left(\hat{\Delta}_{1}, 5,7\right)$ then $d_{4} \geq 6,(-, 16,56,56)$ and $(\Sigma, \tilde{c})=(128,196)$; or if $\left(2, \hat{\Delta}_{1}, 5,7\right)$ then $d_{4} \geq 6,(56,16,56,64)$ and $(\Sigma, \tilde{c})=(192,196)$. It follows that $c^{*}\left(\hat{\Delta}_{2}\right) \leq 0$. This completes the proof of Theorem 1.1.

\section{Proof of Theorems 1.2 and 1.3}

The proof of Theorems 1.2 and 1.3 for $n=4$ and $n=6$ is given in [8] and for $n=5$ in [20] so assume that $n \geq 7$. Let $\mathcal{R}_{n}(k, l, \varepsilon)$ be weakly diagrammatically reducible and so, as mentioned in the introduction, every finite subgroup of $G_{n}(k, l, \varepsilon)$ is conjugate to a subgroup of $H=\left\langle t \mid t^{n}\right\rangle$. It follows that if the exponent sum of $x$ in $t^{3} x^{k} t^{2} x^{\varepsilon(k+l)}$ is not equal to \pm 1 then $x$ has infinite order in $G_{n}(k, l, \varepsilon)$. On the other hand, if $\varepsilon=-1$ and $l=1$ then since the natural map from $H$ to $G_{n}(k, 1,-1)$ has a left inverse and $x$ occurs more than once in the relator, the conclusion of Theorem 3.6 in [5] applies and so $G_{n}(k, 1,-1)$ is infinite.

Let $\varepsilon=1$. It follows from the above paragraph and Theorem 1.1(i) that the only case remaining is $l=0$ and $n \geq 7$. But statement Theorem 1.2(vi) follows from Lemma 2.6.

Let $\varepsilon=-1$. If $l=0$ then clearly $G_{n}(k, 0,-1)$ is infinite so assume that $l \geq 1$. Again it follows from the above and Theorem 1.1(ii)-(iv) that the remaining cases are $l=k$ and 
$n \in\{7,8,9,10,12\}, l=2 k$ and $n \in\{7,8\}$ and $k=2 l$ and $n \in\{7,8\}$. If $l=k>1$ or $l=2 k>2$ or $k=2 l>2$ then $G_{n}(k, l, \varepsilon)$ is infinite by Lemma 2.5. This leaves $(n, k, l) \in$ $\{(7,1,1),(8,1,1),(9,1,1),(10,1,1),(12,1,1),(7,1,2),(8,1,2),(7,2,1),(8,2,1)\}$. But $(7,2,1)$ and $(9,1,1)$ are the exceptional cases and Theorem 1.3(iv) then follows from Lemma 2.4 .

\section{References}

[1] Abd Ghafur Bin Ahmad, Muna A. Al-Mulla and Martin Edjvet, Asphericity of length four relative group presentations, Journal of Algebra and its Applications 16 (2017) 1750076.

[2] Suzana Aldwaik and Martin Edjvet. On the asphericity of a family of positive relative group presentations, Proc. Edin. Math. Soc. 60 (2017) 545-564.

[3] Suzana Aldwaik, Martin Edjvet and Arye Juhasz, Asphericity of positive free product length 4 relative group presentations, Forum Mathematicum 31 (2019) 49-68.

[4] Y. G. Baik, William A. Bogley and Stephen J. Pride, On the asphericity of length four relative group presentations, Int. J. Algebra Comput. 7 (1997) 277-312.

[5] William A. Bogley, Martin Edjvet and Gerald Williams, Aspherical relative presentations all over again, Proceedings of Groups St Andrews in Birmingham 2017, LMS Lecture Note Series 455 Cambridge University Press 169-199.

[6] William A. Bogley and Forrest W. Parker, Cyclically presented groups with length four positive relators, J Group Theory 21 (2018) 911-947.

[7] William A. Bogley and Stephen J. Pride, Aspherical relative presentations, Proc. Edin. Math. Soc. 35 (1992) 1-39.

[8] William A. Bogley and Gerald Williams, Efficient finite groups arising in the study of relative asphericity, Math. Z. 284 (2016) 507-535.

[9] William A. Bogley and Gerald Williams, Coherence, subgroup separability, and metacyclic structures for a class of cyclically presented groups, Journal of Algebra 480 (2017) 266-297.

[10] D. J. Collins and J. Huebschmann, Spherical diagrams and identities among relations, Math. Ann. 261 (1982) 155-183.

[11] Peter J. Davidson, On the asphericity of a family of relative group presentations, Int. J. Algebra Comput. 19 (2009) 159-189.

[12] Martin Edjvet, Equations over groups and a theorem of Higman, Neumann and Neumann, Proc. Lond. Math. Soc. 63 (1991) 563-589.

[13] Martin Edjvet, On the asphericity of one-relator relative presentations, Proc. R. Soc. Edinb., Sect. A 124 (1994) 713-728.

[14] Martin Edjvet and Arye Juhasz, The infinite Fibonacci groups and relative asphericity, Trans. Lond. Math. Soc. 4 (2017) 148-218.

[15] The GAP Group, GAP - Groups, Algorithms and Programming, Version 4.8.7, (2017).

[16] Murray Gerstenhaber and Oscar S. Rothaus, The solution of sets of equations in groups, Proc. Nat. Acad. Sci. U.S.A. 48 (1962) 1531-1533.

[17] James Howie and V. Metaftsis, On the asphericity of length five relative group presentations, Proc. Lond. Math. Soc. 82 (2001) 173-194. 
[18] F. Levin, Solutions of equations over groups, Bull. Amer. Math. Soc. 68 (1962) 603-604.

[19] M. F. Newman, Proving a group infinite, Arch. Math. 54 (1990) 209-211.

[20] S. J. Pride, Groups with presentations in which each defining relator involves exactly two generators, J. London Math. Soc. 36 (1987) 245-256.

[21] M. I. Prishchepov, Asphericity, atoricity and symmetrically presented groups, Comm. Algebra 23 (1995) 5095-5117. 